\title{
Flocks, herds, and schools: A quantitative theory of flocking
}

\author{
John Toner \\ Institute of Theoretical Science, Materials Science Institute, and Department of Physics, \\ University of Oregon, Eugene, Oregon 97403-5203 \\ Yuhai Tu \\ IBM Thomas J. Watson Research Center, P.O. Box 218, Yorktown Heights, New York 10598
}

(Received 16 April 1998)

\begin{abstract}
We present a quantitative continuum theory of "flocking": the collective coherent motion of large numbers of self-propelled organisms. In agreement with everyday experience, our model predicts the existence of an "ordered phase" of flocks, in which all members of even an arbitrarily large flock move together with the same mean velocity $\langle\vec{v}\rangle \neq 0$. This coherent motion of the flock is an example of spontaneously broken symmetry: no preferred direction for the motion is picked out a priori in the model; rather, each flock is allowed to, and does, spontaneously pick out some completely arbitrary direction to move in. By analyzing our model we can make detailed, quantitative predictions for the long-distance, long-time behavior of this "broken symmetry state." The "Goldstone modes" associated with this "spontaneously broken rotational symmetry" are fluctuations in the direction of motion of a large part of the flock away from the mean direction of motion of the flock as a whole. These "Goldstone modes" mix with modes associated with conservation of bird number to produce propagating sound modes. These sound modes lead to enormous fluctuations of the density of the flock, far larger, at long wavelengths, than those in, e.g., an equilibrium gas. Our model is similar in many ways to the Navier-Stokes equations for a simple compressible fluid; in other ways, it resembles a relaxational time-dependent Ginsburg-Landau theory for an $n=d$ component isotropic ferromagnet. In spatial dimensions $d>4$, the long-distance behavior is correctly described by a linearized theory, and is equivalent to that of an unusual but nonetheless equilibrium model for spin systems. For $d<4$, nonlinear fluctuation effects radically alter the long distance behavior, making it different from that of any known equilibrium model. In particular, we find that in $d=2$, where we can calculate the scaling exponents exactly, flocks exhibit a true, long-range ordered, spontaneously broken symmetry state, in contrast to equilibrium systems, which cannot spontaneously break a continuous symmetry in $d=2$ (the "Mermin-Wagner" theorem). We make detailed predictions for various correlation functions that could be measured either in simulations, or by quantitative imaging of real flocks. We also consider an anisotropic model, in which the birds move preferentially in an “easy" (e.g., horizontal) plane, and make analogous, but quantitatively different, predictions for that model as well. For this anisotropic model, we obtain exact scaling exponents for all spatial dimensions, including the physically relevant case $d=3$. [S1063-651X(98)08410-4]
\end{abstract}

PACS number(s): 87.10.+e, 64.60.Cn, 05.60.+w

\section{INTRODUCTION}

A wide variety of nonequilibrium dynamical systems with many degrees of freedom have recently been studied using powerful techniques developed for equilibrium condensed matter physics (e.g., scaling, the renormalization group, etc.). One of the most familiar examples of a many-degree-offreedom, nonequilibrium dynamical system is a large flock of birds. Myriad other examples of the collective, coherent motion of large numbers of self-propelled organisms occur in biology: schools of fish, swarms of insects, slime molds, herds of wildebeest, etc.

Recently, a number of simulations of this phenomenon have been performed [1-3]. Following Reynolds [3], we will use the term "boid" and bird interchangeably for the particles in these simulations. All of these simulations have several essential features in common:

(1) A large number (a "flock") of point particles ("boids") each move over time through a space of dimension $d(=2,3, \ldots)$, attempting at all times to "follow" (i.e., move in the same direction as) its neighbors.
(2) The interactions are purely short ranged: each "boid" only responds to its neighbors, defined as those "boids" within some fixed, finite distance $R_{0}$, which is assumed to be much less than $L$, the size of the "flock."

(3) The "following" is not perfect: the "boids" make errors at all times, which are modeled as a stochastic noise. This noise is assumed to have only short-ranged spatiotemporal correlations.

(4) The underlying model has complete rotational symmetry: the flock is equally likely, a priori, to move in any direction.

The development of a nonzero mean center-of-mass velocity $\langle\vec{v}\rangle$ for the flock as a whole therefore requires spontaneous breaking of a continuous symmetry (namely, rotational).

In an earlier paper [4], we formulated a continuum model for such dynamics of flocking, and obtained some exact results for that model in spatial dimensions $d=2$ (appropriate for the description of the motion of land animals on the Earth's surface). Our most surprising result [4] was that twodimensional moving herds with strictly short-ranged interac- 
tions appear to violate the Mermin-Wagner theorem [5], in that they can acquire long-ranged order, by picking out a consistent direction of motion across an arbitrarily large herd, despite the fact that this involves spontaneously breaking a continuous (rotational) symmetry.

Of course, this result does not, in fact, violate the Mermin-Wagner theorem, since flocks are a nonequilibrium dynamical system. What is fascinating (at least to us) about our result is that the nonequilibrium aspects of the flock dynamics that make the long-distance, long-time behavior of the flock different from that of otherwise analogous equilibrium systems are fundamentally nonlinear, strong-fluctuation effects. Indeed, a "breakdown of linearized hydrodynamics,' analogous to that long known to occur in equilibrium fluids [6] in spatial dimensions $d=2$, occurs in flocks for all $d<4$. This breakdown of linearized hydrodynamics is essential to the very existence of the ordered state in $d=2$. Furthermore, it has dramatic consequences even for $d>2$.

The physics of this breakdown is very simple: above $d$ $=4$, where the breakdown does not occur, information about what is going on in one part of the flock can be transmitted to another part of the flock only by being passed sequentially through the intervening neighbors via the assumed shortranged interactions. Below $d=4$, where the breakdown occurs, this slow, diffusive transport of information is replaced by direct, convective transport: fluctuations in the local velocity of the flock became so large, in these lower dimensions, that the motion of one part of the flock relative to another becomes the principal means of information transport, because it becomes faster than diffusion. There is a sort of "negative feedback," in that this improved transport actually suppresses the very fluctuations that give rise to it, leading to long-ranged order in $d=2$. The purpose of the present paper is to study the properties of the "ordered state" of the flock, i.e., the state in which all members of the flock are moving in the same average direction. Specifically, we will do the following:

(1) We will give the details of the derivation of the results of Ref. [4], and give detailed predictions for numerous correlation functions that can be measured in both experiments and simulations. In particular, we will show that two propagating sound modes exist in flocks, with unusually anisotropic speeds, whose detailed dependence on the direction of propagation we predict, making possible extremely stringent quantitative tests of our theory. We also calculate their attenuations, which show highly anomalous, and strongly anisotropic, scaling.

(2) We will formulate and study the most complete generalization of the model of Ref. [4] for spatial dimensions $d>2$.

(3) We will include the effect of spatial anisotropy (e.g., the fact that birds prefer to fly horizontally rather than vertically) on flock motion.

We describe the flock with coarse-grained density and velocity fields $\rho(\vec{r}, t)$ and $\vec{v}(\vec{r}, t)$, respectively, giving the average number density and velocity of the birds at time $t$ within some coarse-graining distance $\ell_{0}$ of a given position $\vec{r}$ in space. The coarse-graining distance $\ell_{0}$ is chosen to be as small as possible, consistent with being large enough that the averaging can be done sensibly (in particular, $\ell_{0}$ must be greater than the mean interbird distance). Our description is then valid for distances large compared to $\ell_{0}$, and for times $t$ much greater than some microscopic time $t_{0}$, presumably of order $\ell_{0} / v_{T}$, where $v_{T}$ is a typical speed of a bird. Collective motion of the flock as a whole then requires that $\langle\vec{v}(\vec{r}, t)\rangle \neq 0$; where the averaging can be considered an ensemble average, a time average, or a spatial average. Equivalently, long-ranged order must develop for the flock as a whole to move; i.e., the equal-time velocity autocorrelation function:

$$
C(\vec{R}) \equiv\langle\vec{v}(\vec{R}+\vec{r}, t) \cdot \vec{v}(\vec{r}, t)\rangle
$$

must approach a nonzero constant as the separation $|\vec{R}|$ $\rightarrow \infty$; specifically,

$$
C(\vec{R} \rightarrow \infty) \rightarrow|\langle\vec{v}\rangle|^{2}
$$

Thus the average velocity $\langle\vec{v}\rangle$ of the flock is precisely analogous to the order parameter $\langle\vec{s}\rangle$ in a ferromagnetic system, where $\vec{s}$ is a local spin.

Our most dramatic result is that an intrinsically nonequilibrium and nonlinear feature of our model, namely, convection, suppresses fluctuations of the velocity $\vec{v}$ at long wavelengths, making them much smaller than the analogous $\vec{s}$ fluctuations found in ferromagnets, for all spatial dimensions of the flock $d<4$. Specifically, the connected piece $C_{C}(\vec{R})$ of the correlation function $C(\vec{R})$, defined as

$$
C_{C}(\vec{R})=C(\vec{R})-\lim _{\left|\vec{R}^{\prime}\right| \rightarrow \infty} C\left(\vec{R}^{\prime}\right),
$$

which is a measure of the fluctuations, decays to zero much more rapidly, as $|\vec{R}| \rightarrow \infty$, than the analogous correlation function in magnets. Quantitatively, for points whose separation $\vec{R} \equiv \vec{R}_{\perp}$ lies perpendicular to the mean direction of motion of the flock,

$$
C_{C}(\vec{R}) \propto R_{\perp}^{2 \chi},
$$

where the universal "roughness exponent",

$$
\chi=-\frac{1}{5}
$$

exactly, in $d=2$, and is $<1-d / 2$, its value in magnetic systems, for all $d<4$. For $d>4, \chi=1-d / 2$ for flocks as well as for magnets.

The physical mechanism for this suppression of fluctuations is easy to understand: increased fluctuations in the direction of motion of different parts of the flock actually enhance the exchange of information between those different parts. This exchange, in turn, suppresses those very fluctuations, since the interactions between birds tend to make them all move in the same direction.

These nonequilibrium effects also lead to a spatial anisotropy of scaling between the direction along $(\|)$ and those orthogonal to $(\perp)$ the mean velocity $\langle\vec{v}\rangle$. The physical origin of the anisotropy is also simple: if birds make small errors $\delta \theta$ in their direction of motion, their random motion perpendicular to the mean direction of motion $\langle\vec{v}\rangle$ is much larger 
than that along $\langle\vec{v}\rangle$; the former is $\propto \delta \theta$, while the later is proportional to $1-\cos \delta \theta \sim \delta \theta^{2}$. As a result, any equal-time correlation function in the system of any combination of fields crosses over from dependence purely on $\left|\vec{R}_{\perp}\right|$ to dependence purely on $R_{\|}$when

$$
\frac{R_{\|}}{\ell_{0}} \approx\left(\frac{\left|\vec{R}_{\perp}\right|}{\ell_{0}}\right)^{\zeta}
$$

where $\ell_{0}$ is the bird interaction range.

The universal anisotropy exponent

$$
\zeta=\frac{3}{5},
$$

exactly in $d=2$, and is $<1$ for all $d<4$.

In particular, the connected, equal-time, velocity autocorrelation function $C_{C}(\vec{R})$ obeys the scaling law

$$
C_{C}(\vec{R})=\left|\vec{R}_{\perp}\right|^{2 \chi} f_{v}\left(\frac{\left(R_{\|} / \ell_{0}\right)}{\left(\left|\vec{R}_{\perp}\right| / \ell_{0}\right)^{\zeta}}\right)
$$

where $f_{v}(x)$ is a universal scaling function. We have been unable to calculate this scaling function, even in $d=2$ where we know the exponents exactly. However, the scaling form (1.8) immediately implies that

$$
C_{C}(\vec{R}) \propto R_{\|}^{2 \chi / \zeta}, \quad \text { when } \quad R_{\|} / \ell_{0} \gg\left(\vec{R}_{\perp} / \ell_{0}\right)^{\zeta} .
$$

So far, our discussion has focused on velocity fluctuations. The density $\rho(\vec{R}, t)$ shows huge fluctuations as well: indeed, at long wavelengths, the fluctuations of the density of

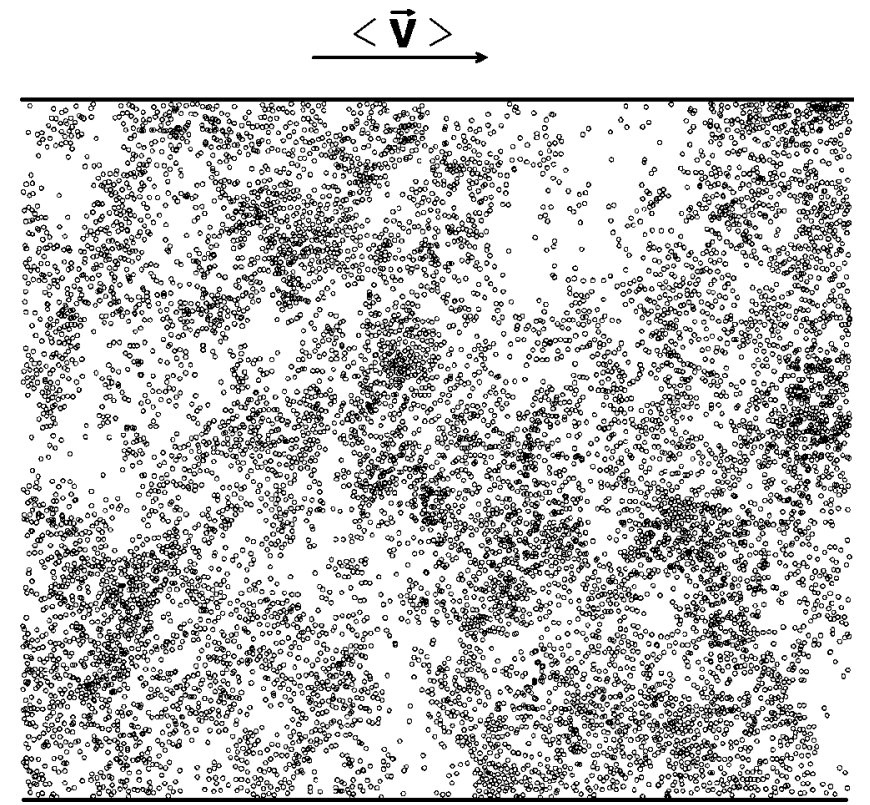

FIG. 1. A snapshot of a simulated flock that has reached a statistically steady state. Note the enormous fluctuations in the density. Quantitatively, the statistics of the spatial Fourier transform $C_{\rho}(\vec{q})$ obtained from this picture agree with our quantitative prediction equation (1.10).

birds in a flock become infinitely bigger than those in a fluid or an ideal gas. This fact is obvious to the eye in a picture of a flock (see Fig. 1). Quantitatively, we predict that the spatially Fourier transformed, equal-time density-density correlation function $C_{\rho}(\vec{q}) \equiv\left\langle|\rho(\vec{q}, t)|^{2}\right\rangle$ obeys the scaling law

$$
C_{\rho}(\vec{q})=\frac{q_{\perp}^{3-d-\zeta-2 \chi}}{q^{2}} f_{\rho}\left(\frac{q_{\|} \ell_{0}}{\left(q_{\perp} \ell_{0}\right)^{\zeta}}\right) Y\left(\theta_{q}^{\vec{q}}\right) \propto \begin{cases}q_{\perp}^{1-d-\zeta-2 \chi}, & q_{\|} \ll q_{\perp} \\ q_{\|}^{-2} q_{\perp}^{3-d-\zeta-2 \chi}, & \left(\ell_{0} q_{\perp}\right)^{\zeta} \gg \ell_{0} q_{\|} \gg q_{\perp} \ell_{0} \\ q_{\|}^{-3+(1-d-2 \chi / \zeta)} q_{\perp}^{2}, & \left(q_{\perp} \ell_{0}\right)^{\zeta} \ll q_{\|} \ell_{0},\end{cases}
$$

where $Y\left(\theta_{\vec{q}}\right)$ is a finite, nonvanishing, $O(1)$ function of the angle $\theta_{\vec{q}}$ between the wave vector $\vec{q}$ and the direction of mean flock motion, $q_{\|,}$and $\vec{q}_{\perp}$ are the wave vectors parallel and perpendicular to the broken symmetry direction, and $q_{\perp}=\left|\vec{q}_{\perp}\right|$.

In $d=2, \zeta=\frac{3}{5}$ and $\chi=-\frac{1}{5}$, so

$$
C_{\rho}(\vec{q})=\frac{q_{\perp}^{(4 / 5)}}{q^{2}} f_{\rho}\left(\frac{q_{\|} \ell_{0}}{\left(q_{\perp} \ell_{0}\right)^{(3 / 5)}}\right) Y\left(\theta_{q}^{\vec{q}}\right) \propto \begin{cases}q_{\perp}^{-(6 / 5)}, & q_{\|} \ll q_{\perp} \\ q_{\|}^{-2} q_{\perp}^{(4 / 5)}, & \left(\ell_{0} q_{\perp}\right)^{(3 / 5)} \gg \ell_{0} q_{\|} \gg q_{\perp} \ell_{0} \\ q_{\|}^{-4} q_{\perp}^{2}, & \left(q_{\perp} \ell_{0}\right)^{(3 / 5)} \ll q_{\|} \ell_{0} .\end{cases}
$$

The most important thing to note about $C_{\rho}(\vec{q})$ is that it diverges as $|\vec{q}| \rightarrow 0$, unlike $C_{\rho}(\vec{q})$ for, say, a simple fluid or gas, or, indeed, for any equilibrium condensed matter system, which goes to a finite constant (the compressibility) as $|\vec{q}| \rightarrow 0$.

This correlation function should be extremely easy to measure in simulations, and in experiments on real herds or flocks, in which, say, video tape allows one to measure the positions $\vec{r}_{i}(t)$ of all the birds (labeled by $i$ ) in the flock at a variety of times $t$. The recipe is simple:
(1) Calculate the complex numbers

$$
\rho(\vec{q}, t)=\sum_{i} e^{i \vec{q} \cdot \vec{r}_{i}(t)}
$$

for a variety of $\vec{q}$ 's.

(2) Average the squared magnitude of this number over time. The result is $C_{\rho}(\vec{q})$. 


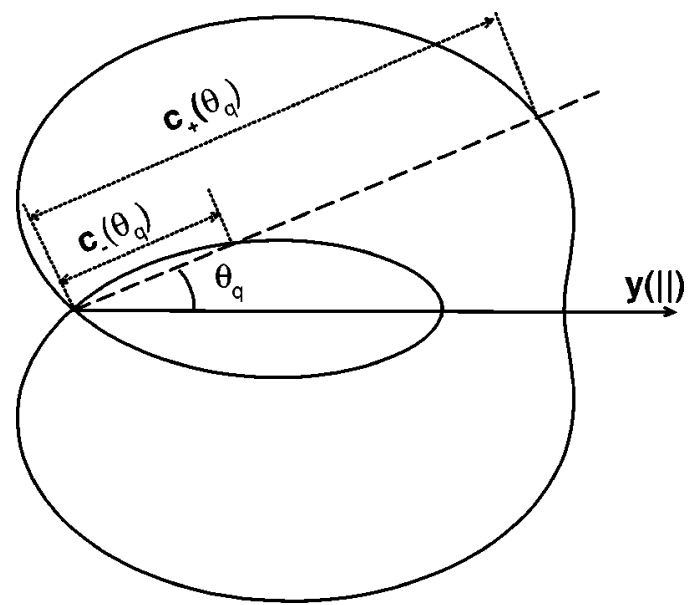

FIG. 2. Polar plot of the direction-dependent sound speeds $c_{ \pm}\left(\theta_{\vec{q}}\right)$, with the horizontal axis along the direction of mean flock motion.

Time-dependent correlation functions of $\rho$ and $\vec{v}$ in flocks also show interesting anomalous scaling behavior. However, it is not so simple to summarize as the equal-time correlation functions. Indeed, time-dependent correlation functions (or, equivalently, their spatiotemporal Fourier transforms) do not have a simple scaling form. This is because the collective normal modes of the flock consist of propagating, damped longitudinal "sound" modes (i.e., density waves), as well as, in $d>2$, shear modes. The sound modes exhibit two different types of scaling: the period $T$ of a wave is proportional to its wavelength $\lambda$ (the constant of proportionality being the inverse sound speed); while the lifetime $\tau$ of the mode is proportional to $\lambda^{z}$, with $z$ being another universal exponent. In most systems (e.g., fluids, crystals) exhibiting sound modes, $z=2$, corresponding to conventional diffusive or viscous damping [6]. In flocks, however, we find

$$
z=\frac{6}{5}, \quad d=2,
$$

and $z<2$ for all $d<4$, for sound modes propagating orthogonal to the mean direction of flock motion. That is, sound modes are much more heavily damped at long wavelengths in flocks than in most [7] equilibrium condensed matter systems.

The full dispersion relation for the sound modes is

$$
\omega_{ \pm}=c_{ \pm}\left(\theta_{\vec{q}}\right) q-i q_{\perp}^{z} f_{ \pm}\left(\frac{q_{\|} \ell_{0}}{\left(q_{\perp} \ell_{0}\right)^{\zeta}}\right),
$$

where $\theta_{\vec{q}}$ is the angle between $\vec{q}$ and $\langle\vec{v}\rangle$, and the directiondependent sound speeds $c_{ \pm}\left(\theta_{q}^{\vec{q}}\right)$ are given by Eq. (4.11) of Sec. IV, with $\gamma$ and $\sigma_{1}$ flock-dependent parameters and $\rho_{0}$ the mean number density of "birds" in the flock. A polar plot of these sound speeds is given in Fig. 2. The exponent $\zeta$ is the universal anisotropy exponent described earlier, and $f_{ \pm}(x)$ are universal scaling functions that we have been unable to calculate. However, we do know some of their limits:

$$
f_{ \pm}(x \rightarrow 0) \rightarrow \text { const }>0 ; \quad f_{ \pm}(x \rightarrow \infty) \propto x^{z / \zeta} .
$$

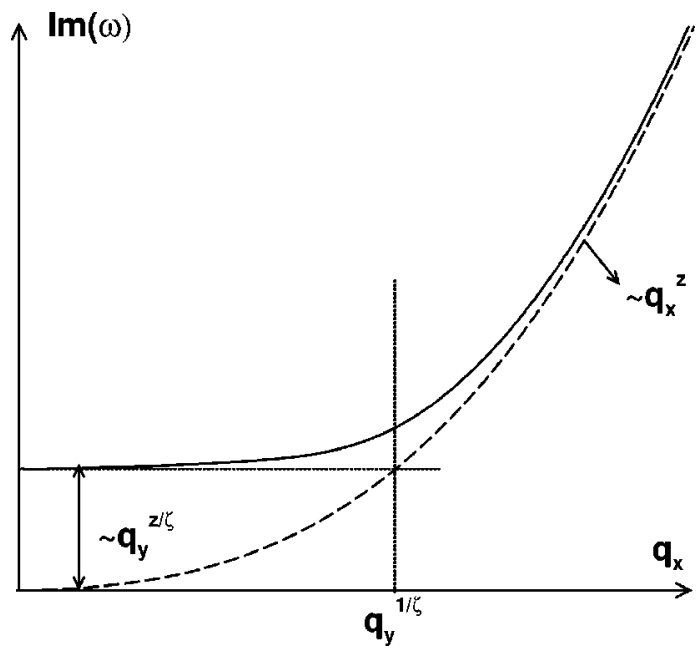

FIG. 3. Plot of the damping $\operatorname{Im} \omega$ vs $q_{x} \equiv\left|\vec{q}_{\perp}\right|$ where $\vec{q}_{\perp}$ is the projection of wave vector $\vec{q}$ perpendicular to the direction of the mean flock velocity $\langle\vec{v}\rangle$ for fixed projection $q_{y}$ of $\vec{q}$ parallel to $\langle\vec{v}\rangle$. Note that, for small $|\vec{q}|$, the crossover between $\operatorname{Im} \omega \propto q_{x}^{z}$ and $\operatorname{Im} \omega \propto q_{y}^{z / \zeta}$ occurs only for directions of propagation $\hat{q}$ very nearly parallel to the mean flock velocity $\langle\vec{v}\rangle$, since $\zeta<1$.

Note that this last result implies that the lifetime $\tau$ of the wave is $\propto q_{\|}^{-z / \zeta}$ for $q_{\|} \ell_{0} \gg\left(q_{\perp} \ell_{0}\right)^{\zeta}$ and $|\vec{q}| \rightarrow 0$; this only happens for directions of propagation very nearly parallel to $\langle\vec{v}\rangle$. Note also that in $d=2$ where $z=\frac{6}{5}$ and $\zeta=\frac{3}{5}, z / \zeta=2$ and the damping is conventional for sound modes propagating parallel to the mean motion of the flock. For all other directions of propagation, however, it is unconventional, and characterized by $z=\frac{6}{5}$.

This behavior of the damping (i.e., $\operatorname{Im} \omega$ ), is summarized in Fig. 3. For $d>2$, the "hydrodynamic" mode structure also includes $d-2$ "hyperdiffusive" shear modes, with identical dispersion relations

$$
\omega_{s}=\gamma q_{\|}-i q_{\perp}^{z} f_{s}\left(\frac{q_{\|} \ell_{0}}{\left(q_{\perp} \ell_{0}\right)^{\zeta}}\right) .
$$

The dispersion relations for $\omega_{ \pm}$and $\omega_{s}$ can be directly probed by measuring the spatiotemporally Fourier transformed density-density and velocity-velocity autocorrelation functions

$$
\begin{gathered}
C_{\rho}(\vec{q}, \omega) \equiv\left\langle|\rho(\vec{q}, \omega)|^{2}\right\rangle, \\
C_{i j}(\vec{q}, \omega) \equiv\left\langle v_{i}(\vec{q}, \omega) v_{j}(-\vec{q},-\omega)\right\rangle,
\end{gathered}
$$

respectively. Experimentally, or in simulations, $C_{\rho}(\vec{q}, \omega)$ can be calculated by temporally Fourier transforming the spatially Fourier transformed density (1.12):

$$
\rho_{n}(\vec{q}, \omega)=\sum_{t=n \tau}^{(n+1) \tau} \rho_{n}(\vec{q}, t) e^{-i \omega t}, \quad n=0,1,2, \ldots
$$

over a set of long "bins" of time intervals of length $\tau \gg t_{0}$ (the "microscopic" time step), and then averaging the squared magnitude $|\rho(\vec{q}, \omega)|^{2}$ over bins: 


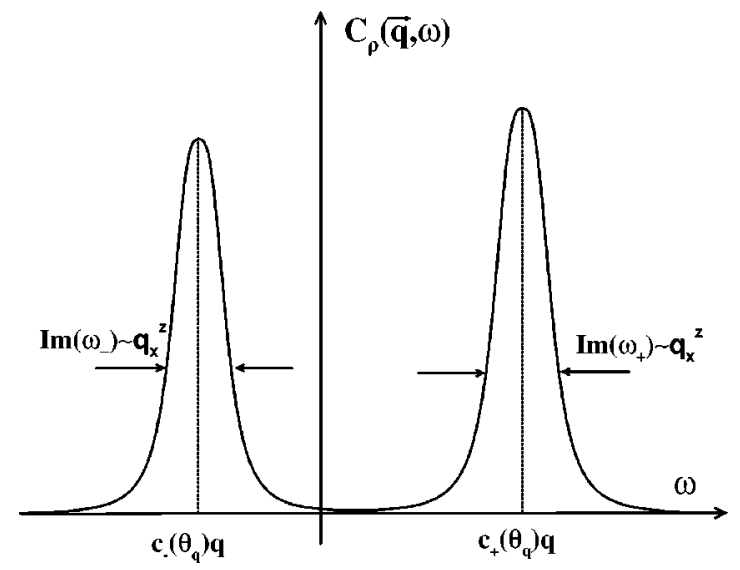

FIG. 4. Plot of the spatiotemporally Fourier-transformed density correlation function $C_{\rho}(\vec{q}, \omega)$ vs $\omega$ for fixed $\vec{q}$. It shows two sharp asymmetrical peaks at $\omega=c_{ \pm}\left(\theta_{\vec{q}}\right) q$ associated with the sound modes of the flock, where $c_{ \pm}\left(\theta_{\vec{q}}\right)$ are the sound mode speeds. The widths of those peaks are the second mode dampings $\operatorname{Im} \omega_{ \pm}\left(\theta_{\vec{q}}\right)$ $\propto q_{\perp}^{z} f_{ \pm}\left[q_{\|} \ell_{0} /\left(q_{\perp} \ell_{0}\right)^{\zeta}\right]$.

$$
C_{\rho}(\vec{q}, \omega) \equiv \sum_{n=0}^{n_{\max }} \frac{|\rho(\vec{q}, \omega)|^{2}}{n_{\max }}
$$

Closed form expressions for these correlation functions in terms of the scaling functions $f_{L}$ and $f_{s}$ are given in Sec. V. Although these expressions look quite complicated, the behavior they predict is really quite simple, as illustrated in Fig. 4, where $C_{\rho}$ is plotted as a function of $\omega$ for fixed $q$. As shown there, $C_{\rho}$ has two sharp peaks at $\omega=c_{ \pm}\left(\theta_{q}\right) q$, of width $\propto q_{\perp}^{z} f_{L}\left[q_{\|} \ell_{0} /\left(q_{\perp} \ell_{0}\right)^{\zeta}\right]$ and height $\propto q_{\perp}^{-(2 \chi x+z+3 \zeta+d-3)} g\left[q_{\|} \ell_{0} /\left(q_{\perp} \ell_{0}\right)^{\zeta}\right]$. Thus, $c_{ \pm}\left(\theta_{q}\right)$ can be simply extracted from the position of the peaks, while the exponents $\chi, z$, and $\zeta$ can be determined by comparing their widths and heights for different $\vec{q}$ 's.

The scaling properties of the flock are completely summarized by the universal exponents $z, \zeta$, and $\chi$. In $d=2$, our predictions for these exponents are

$$
z=\frac{6}{5}, \quad \zeta=\frac{3}{5}, \quad \chi=-\frac{1}{5} .
$$

These results are exact and universal for all flocks with the simple symmetries we discussed at the outset.

For $d \neq 2$, the situation is less clear. We have performed a one-loop, $4-\epsilon$ expansion to attempt to calculate these exponents, and find that, to this order, the model appears to have a fixed line with continuously varying exponents $z, \zeta$, and $\chi$. Whether this is an artifact of our one-loop calculation, or actually happens, is unclear. A two-loop calculation might clarify matters, but would be extremely long and tedious. (One loop was hard enough.)

The origin of this complication is an additional convective nonlinearity [8] not discussed in Ref. [4]. This new term (whose coefficient is a parameter we call $\lambda_{2}$ ) is unrenormalized at one loop order, leading to the apparent fixed line at that order. In two dimensions, this extra term can be written as a total derivative, and can be absorbed into the nonlinear term considered in Ref. [4]. Hence, in $d=2$, the results of [4] are sound. In $d>2$, however, this new term has a different structure, and could, if it does not renormalize to zero,

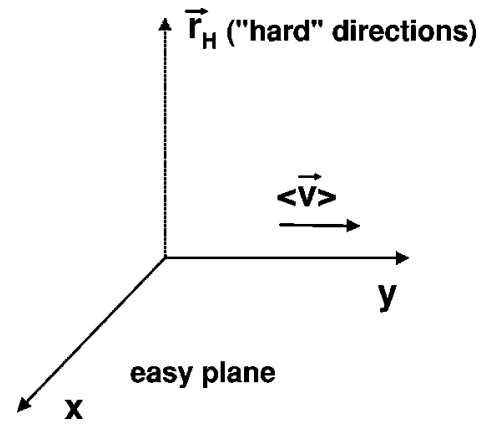

FIG. 5. Geometry of the anisotropic model. Birds prefer to fly in the "easy" $x-y$ plane. We take their (spontaneously chosen) direction of motion within that plane to be $y$. The in-plane direction perpendicular to that is $x$. In general $d$, there are $d-2$ "hard" directions $\vec{r}_{H}$ perpendicular to this easy plane. The anisotropy of scaling is between $x$ and the other $d-1$ directions $y, \vec{r}_{H}$.

change the exponents $\chi, z$, and $\zeta$. Since $\lambda_{2}$ does not renormalize at one-loop order, all we can say at this point is that there are three possibilities:

(1) At higher order, $\lambda_{2}$ renormalizes to zero. If this is the case, we can show that

$$
z=\frac{2(d+1)}{5}, \quad \zeta=\frac{d+1}{5}, \quad \chi=\frac{3-2 d}{5}
$$

exactly, for all $d$ in the range $2 \leqslant d \leqslant 4$. Note that these results linearly interpolate between the equilibrium results $z$ $=2, \zeta=1$, and $\chi=1-d / 2$ in $d=4$, and our $2 d$ results $z$ $=\frac{6}{5}, \zeta=\frac{3}{5}$, and $\chi=-\frac{1}{5}$ in $d=2$.

(2) At higher order, $\lambda_{2}$ grows upon renormalization and reaches a nonzero fixed point value $\lambda_{2}^{*}$ at some new fixed point that differs from the $\lambda_{2}=0$ fixed point we have studied previously, at which Eq. (1.21) holds. The exponents $\chi, z$, and $\zeta$ would still be universal (i.e., depend only on the dimension of space $d$ ) for all flocks in this case, but those universal values would be different from Eq. (1.21).

(3) $\lambda_{2}$ is unrenormalized to all orders. Should this happen, $\lambda_{2}$ would parametrize a fixed line, with continuously varying values of the exponents $z, \chi$, and $\zeta$.

We reiterate: we do not know which of the above possibilities holds for $d>2$. However, whichever holds is universal; that is, only one of the three possibilities above applies to all flocks. We do not, however, know which one that is.

We also study an anisotropic model for flocking, which incorporates the possibility that birds are averse to flying in certain directions (e.g., straight up or straight down). In particular, we consider the case in which, for arbitrary spatial dimensions $d \geqslant 2$, there is an easy plane for motion (i.e., a $d_{e}=2$ dimensional subspace of the full $d$-dimensional space). In this case, the relevant pieces of the $\lambda_{2}$ vertex become a total derivative, and can be absorbed into the nonlinear term considered in Ref. [4], for all spatial dimensions $d$, not just $d=2$ as in the isotropic model. Hence, we are able to obtain exact exponents for this problem for all spatial dimensions $d$, not just $d=2$.

We again find anisotropic, anomalous scaling for $d<4$. The anisotropy of scaling is between the direction in the easy plane (call it $x$ ) perpendicular to the mean direction of mo- 
tion (call it $y$; which, of course, also lies in the easy plane), and all $d-1$ other directions, including $y$ (see Fig. 5). That is, the equal time, velocity-velocity autocorrelation function obeys the scaling law

$$
C_{v}(\vec{R})=x^{2} \chi_{v}\left(\frac{\left(y / \ell_{0}\right)}{\left(x / \ell_{0}\right)^{\zeta}}, \quad \frac{\left|\vec{r}_{H}\right| / \ell_{0}}{\left(x / \ell_{0}\right)^{\zeta}}\right),
$$

where $\vec{r}_{H}$ denotes the $d-2$ components of $\vec{r}$ in the "hard" directions orthogonal to the easy plane, with the scaling exponents $\chi$ and $\zeta$ given by

$$
\begin{gathered}
\chi=\frac{1-d}{7-d}, \\
\zeta=\frac{3}{7-d}
\end{gathered}
$$

exactly, for all spatial dimensions $d$ in the range $2 \leqslant d \leqslant 4$. For $d>4, \chi=1-d / 2$ and $\zeta=1$, as in the isotropic case, while in $d=2$, where the model becomes identical to the isotropic model (the easy plane of motion being the entire space in that case), we again recover the isotropic results, $\zeta$ $=\frac{3}{5}$, and $\chi=-\frac{1}{5}$. For the physical case $d=3$, we have

$$
\chi(d=3)=-\frac{1}{2}, \quad \zeta(d=3)=\frac{3}{4} .
$$

The Fourier-transformed, equal-time density $(\rho-\rho)$ correlation function $C_{\rho}(\vec{q})$ also obeys a scaling law

$$
\begin{aligned}
C_{\rho}(\vec{q})= & q_{x}^{1-2 \chi-(d-1) \zeta}\left(q_{x}^{2}+q_{y}^{2}\right)^{-1} \\
& \times f_{\rho}^{A}\left(\frac{q_{y} \ell_{0}}{\left(q_{x} \ell_{0}\right)^{\zeta}}, \frac{\left|\vec{q}_{H}\right| \ell_{0}}{\left(q_{x} \ell_{0}\right)^{\zeta}}\right) Y_{a}\left(\theta_{x y}\right),
\end{aligned}
$$

where $Y_{a}\left(\theta_{x y}\right)$ is a finite, nonzero, $O(1)$ function of the angle $\theta_{x y}=\tan ^{-1}\left(q_{x} / q_{y}\right)$, and the scaling function $f_{\rho}^{A}$ follows

$$
f_{\rho}^{A}\left(\frac{q_{y} \ell_{0}}{\left(q_{x} \ell_{0}\right)^{\zeta}}, \frac{\left|\vec{q}_{H}\right| \ell_{0}}{\left(q_{x} \ell_{0}\right)^{\zeta}}\right) \propto \begin{cases}\text { const, } & \ell_{0}^{2}\left(q_{y}^{2}+\nu\left|\vec{q}_{H}\right|^{2}\right) \ll\left(\ell_{0} q_{x}\right)^{\zeta} \\ \left(\frac{q_{x}^{2 \zeta}}{q_{y}^{2}+\nu\left|\vec{q}_{H}\right|^{2}}\right)^{[1+2 \chi+(d-1) \zeta] / 2 \zeta} & , \quad \ell_{0}^{2}\left(q_{y}^{2}+\nu\left|\vec{q}_{H}\right|^{2}\right) \gg\left(\ell_{0} q_{x}\right)^{\zeta},\end{cases}
$$

where $\ell_{0}$ is a "microscopic" length (of the order the interbird distance) and $\nu$ a dimensionless nonuniversal constant of order unity.

Finally, the hydrodynamic mode structure of this anisotropic flock consists of a pair of propagating longitudinal sound modes, with dispersion relation given, in the coordinate system of Fig. (5), by

$$
\omega=c_{ \pm}\left(\theta_{\vec{q}}, \phi_{q}\right) q-i q_{x}^{z} f_{A}\left(\frac{q_{y} \ell_{0}}{\left(q_{x} \ell_{0}\right)^{\zeta}}, \quad \frac{\left|\vec{q}_{H}\right| \ell_{0}}{\left(q_{x} \ell_{0}\right)^{\zeta}}\right),
$$

where $f_{A}$ is a universal scaling function, $c_{ \pm}\left(\theta_{\vec{q}}, \phi_{\vec{q}}\right)$ is given by Eq. (6.22) of Sec. 6 and the dynamical exponent

$$
z=2 \zeta=\frac{6}{7-d}=\frac{3}{2},
$$

where the last equality holds in $d=3$. Note that this value of $z$ again reduces to that of the isotropic model in $d=2$, and $d=4$.

The spatiotemporally Fourier-transformed density-density correlation function $\left\langle|\rho(\vec{q}, \omega)|^{2}\right\rangle$ has the same structure as that illustrated for the isotropic problem in Fig. 4, with the modification that $q_{\perp}$ is replaced by $q_{x}$, and the scaling function $f_{L}$ is replaced by $f_{A}$. The detailed expression for $\left\langle|\rho(\vec{q}, \omega)|^{2}\right\rangle$ is given by Eq. (6.31) of Sec. VI.

The remainder of this paper is organized as follows: In Sec. II, we formulate the isotropic model. In Sec. III, we specialize this model to the "broken symmetry" state, in which the flock is moving with a nonzero mean speed $\langle\vec{v}\rangle$. In Sec. IV, we linearize the broken symmetry state model, and calculate the correlation functions and scaling laws in this linear approximation. In Sec. V, we study the anharmonic corrections in the broken symmetry state, show that they diverge in spatial dimensions $d<4$, derive the new scaling laws that result in that case, calculate the exact exponents in $d=2$, and discuss the difficulties that prevent us from obtaining these exponents for $2<d<4$. In Sec. VI, we repeat all of the above for the anisotropic model. In Sec. VII, we describe in some detail how our predictions might be tested experimentally, both by observations of real flocks of living organisms, and in simulations. And, finally, in Sec. VIII, we discuss some of the open questions remaining in this problem, and suggest some possible directions for future research.

\section{THE ISOTROPIC MODEL}

In this section, we formulate our model for isotropic flocks. As discussed in the Introduction, the system we wish to model is any collection of a large number $N$ of organisms (hereafter referred to as "birds") in a $d$-dimensional space, with each organism seeking to move in the same direction as its immediate neighbors.

We further assume that each organism has no "compass;' i.e., no intrinsically preferred direction in which it wishes to move. Rather, it is equally happy to move in any direction picked by its neighbors. However, the navigation of each organism is not perfect; it makes some errors in at- 
tempting to follow its neighbors. We consider the case in which these errors have zero mean; e.g., in two dimensions, a given bird is no more likely to err to the right than to the left of the direction picked by its neighbors. We also assume that these errors have no long temporal correlations; e.g., a bird that has erred to the right at time $t$ is equally likely to err either left or right at a time $t^{\prime}$ much later than $t$.

Although the continuum model we propose here will describe the long-distance behavior of any flock satisfying the symmetry conditions we shall specify in a moment, it is instructive to first consider an explicit example: the automaton studied by Vicsek et al. [1]. In this discrete time model, a number of boids labeled by $i$ in a two-dimensional plane with positions $\left\{\vec{r}_{i}(t)\right\}$ at integer time $t$, each chooses the direction it will move on the next time step (taken to be of duration $\Delta t=1$ ) by averaging the directions of motion of all of those birds within a circle of radius $R_{0}$ (in the most convenient units of length $R_{0}=1$ ) on the previous time step (updating is simultaneous). The distance $R_{0}$ is assumed to be $\ll L$, the size of the flock. The direction the bird actually moves on the next time step differs from the above described direction by a random angle $\eta_{i}(t)$, with zero mean and standard deviation $\Delta$. The distribution of $\eta_{i}(t)$ is identical for all birds, time independent, and uncorrelated between different birds and different time steps. Each bird then, on the next time step, moves in the direction so chosen a distance $v_{0} \Delta t$, where the speed $v_{0}$ is the same for all birds.

To summarize, the rule for bird motion is

$$
\begin{gathered}
\theta_{i}(t+1)=\left\langle\theta_{j}(t)\right\rangle+\eta_{i}(t), \\
\vec{r}_{i}(t+1)=\vec{r}_{i}(t)+v_{0}[\cos \theta(t+1), \sin \theta(t+1)], \\
\left\langle\eta_{i}(t)\right\rangle=0, \\
\left\langle\eta_{i}(t) \eta_{j}\left(t^{\prime}\right)\right\rangle=\Delta \delta_{i j} \delta_{t t^{\prime}},
\end{gathered}
$$

where the average in Eq. (2.1) is over all birds $j$ satisfying

$$
\left|\vec{r}_{j}(t)-\vec{r}_{i}(t)\right|<R_{0}
$$

and $\theta_{i}(t)$ is the angle of the direction of motion of the $i$ th bird (relative to some fixed reference axis) on the time step that ends at $t$. The flock evolves through the iteration of this rule. Note that the "neighbors" of a given bird may change on each time step, since birds do not, in general, move in exactly the same direction as their neighbors.

This model, though simple to simulate, is quite difficult to treat analytically. Our goal in our previous work [4] and this paper is to capture the essential physics of this model in a continuum, "hydrodynamic" description of the flock. Clearly, some short-ranged details must be lost in such a description. However, as in hydrodynamic descriptions of equilibrium systems [6], as well as many recent treatments [9] of nonequilibrium systems, our hope is that our continuum approach can correctly reproduce the long-distance, long-time properties of the class of systems we wish to study. This hope is justified by the notion of universality: all "microscopic models" (in our case, different specifications for the exact laws of motion for an individual bird) that have the same symmetries and conservation laws should have the same long-distance behavior. This belief can be justified by our renormalization-group treatment of the continuum model.

So, given this lengthy preamble, what are the symmetries and conservation laws of flocks?

The only symmetry of the model is rotation invariance: since the "birds" lack a compass, all directions of space are equivalent. Thus, the "hydrodynamic" equations of motion we write down cannot have built into them any special direction picked "a priori"; all directions must be spontaneously picked out by the motion and spatial structure of the flock. As we shall see, this symmetry severely restricts the allowed terms in the equation of motion.

Note that the model does not have Galilean invariance: changing the velocities of all the birds by some constant boost $\vec{v}_{b}$ does not leave the model invariant. Indeed, such a boost is impossible in a model that strictly obeys Vicsek's rules, since the speeds of all the birds will not remain equal to $v_{0}$ after the boost. One could image relaxing this constraint on the speed, and allowing birds to occasionally speed up or slow down, while tending on average to move at speed $v_{0}$. Then the boost just described would be possible, but clearly would change the subsequent evolution of the flock.

Another way to say this is that birds move through a resistive medium, which provides a special Galilean reference frame, in which the dynamics are particularly simple, and different from those in other reference frames. Since real organisms in flocks always move through such a medium (birds through the air, fish through the sea, wildebeest through the arid dust of the Serengeti), this is a very realistic feature of the model.

As we shall see shortly, this lack of Galilean invariance allows terms in the hydrodynamic equations of birds that are not present in, e.g., the Navier-Stokes equations for a simple fluid, which must be Galilean invariant, due to the absence of a luminiferous ether.

The sole conservation law for flocks is conservation of birds: we do not allow birds to be born or die "on the wing."

In contrast to the Navier-Stokes equation, there is no conservation of momentum. This is, ultimately, a consequence of the absence of Galilean invariance.

Having established the symmetries and conservation laws constraining our model, we need now to identify the hydrodynamic variables. They are the coarse-grained bird velocity field $\vec{v}(\vec{r}, t)$ and the coarse-grained bird density $\rho(\vec{r}, t)$. The field $\vec{v}(\vec{r}, t)$, which is defined for all $\vec{r}$, is a suitable weighted average of the velocities of the individual birds in some volume centered on $\vec{r}$. This volume is big enough to contain enough birds to make the average well behaved, but should have a spatial linear extent of no more than a few "microscopic" lengths (i.e., the interbird distance, or by a few times the interaction range $R_{0}$ ). By suitable weighting, we seek to make $\vec{v}(\vec{r}, t)$ fairly smoothly varying in space.

The density $\rho(\vec{r}, t)$ is similarly defined, being just the number of particles in a coarse-graining volume, divided by that volume.

The exact prescription for the coarse graining should be unimportant, as long as $\rho(\vec{r}, t)$ is normalized so as to obey 
the "sum rule" that its integral over any macroscopic volume (i.e., any volume large compared with the aforementioned microscopic lengths) be the total number of birds in that volume. Indeed, the coarse-graining description just outlined is the way that one imagines, in principle, going over from a description of a simple fluid in terms of equations of motion for the individual constituent molecules to the continuum description of the Navier-Stokes equation.

We will also follow the historical precedent of the NavierStokes [6] equation by deriving our continuum, longwavelength description of the flock not by explicitly coarse graining the microscopic dynamics (a very difficult procedure in practice), but, rather, by writing down the most general continuum equations of motion for $\vec{v}$ and $\rho$ consistent with the symmetries and conservation laws of the problem. This approach allows us to bury our ignorance in a few phenomenological parameters (e.g., the viscosity in the NavierStokes equation) whose numerical values will depend on the detailed microscopic rules of individual bird motion. What terms can be present in the EOM's, however, should depend only on symmetries and conservation laws, and not on the microscopic rules.

To reduce the complexity of our equations of motion still further, we will perform a spatial-temporal gradient expansion, and keep only the lowest-order terms in gradients and time derivatives of $\vec{v}$ and $\rho$. This is motivated and justified by our desire to consider only the long-distance, long-time properties of the flock. Higher-order terms in the gradient expansion are "irrelevant": they can lead to finite "renormalization" of the phenomenological parameters of the long-wavelength theory, but cannot change the type of scaling of the allowed terms.

With this lengthy preamble in mind, we now write down the equations of motion:

$$
\begin{gathered}
\partial_{t} \vec{v}+\lambda_{1}(\vec{v} \cdot \vec{\nabla}) \vec{v}+\lambda_{2}(\vec{\nabla} \cdot \vec{v}) \vec{v}+\lambda_{3} \vec{\nabla}\left(|\vec{v}|^{2}\right) \\
=\alpha \vec{v}-\beta|\vec{v}|^{2} \vec{v}-\vec{\nabla} P+D_{B} \vec{\nabla}(\vec{\nabla} \cdot \vec{v}) \\
+D_{T} \nabla^{2} \vec{v}+D_{2}(\vec{v} \cdot \vec{\nabla})^{2} \vec{v}+\vec{f} \\
P=P(\rho)=\sum_{n=1}^{\infty} \sigma_{n}\left(\rho-\rho_{0}\right)^{n} \\
\frac{\partial \rho}{\partial t}+\nabla \cdot(\vec{v} \rho)=0
\end{gathered}
$$

where $\beta, D_{B}, D_{2}$, and $D_{T}$ are all positive, and $\alpha<0$ in the disordered phase and $\alpha>0$ in the ordered state (in meanfield theory). The origin of the various terms is as follows: the $\lambda$ terms on the left-hand side of Eq. (2.6) are the analogs of the usual convective derivative of the coarse-grained velocity field $\vec{v}$ in the Navier-Stokes equation. Here the absence of Galilean invariance allows all three combinations of one spatial gradient and two velocities that transform like vectors; if Galilean invariance did hold, it would force $\lambda_{2}$ $=\lambda_{3}=0$ and $\lambda_{1}=1$. However, Galilean invariance does not hold, and so all three coefficients are nonzero phenomenological parameters whose nonuniversal values are determined by the microscopic rules. The $\alpha$ and $\beta$ terms simply make the local $\vec{v}$ have a nonzero magnitude $(=\sqrt{\alpha / \beta})$ in the ordered phase, where $\alpha>0 . D_{L, 1,2}$ are the diffusion constants (or viscosities) reflecting the tendency of a localized fluctuation in the velocities to spread out because of the coupling between neighboring "birds." The $\vec{f}$ term is a random driving force representing the noise. We assume it is Gaussian with white noise correlations:

$$
\left\langle f_{i}(\vec{r}, t) f_{j}\left(\overrightarrow{r^{\prime}}, t^{\prime}\right)\right\rangle=\Delta \delta_{i j} \delta^{d}\left(\vec{r}-\overrightarrow{r^{\prime}}\right) \delta\left(t-t^{\prime}\right),
$$

where $\Delta$ is a constant, and $i, j$ denote Cartesian components. Finally, $P$ is the pressure, which tends to maintain the local number density $\rho(\vec{r})$ at its mean value $\rho_{0}$, and $\delta \rho=\rho$ $-\rho_{0}$.

The final equation (2.8) is just conservation of bird number (we do not allow our birds to reproduce or die "on the wing', ).

Symmetry allows any of the phenomenological coefficients $\lambda_{i}, \alpha, \sigma_{n}, \beta, D_{i}$ in Eqs. (2.6) and (2.7) to be functions of the squared magnitude $|\vec{v}|^{2}$ of the velocity, and of the density $\rho$ as well.

\section{THE BROKEN SYMMETRY STATE}

We are mainly interested in the symmetry broken phase, specifically in whether fluctuations around the symmetry broken ground state destroy it (as in the analogous phase of the 2D $X Y$ model). For $\alpha>0$, we can write the velocity field as $\vec{v}=v_{0} \hat{x}_{\|}+\overrightarrow{\delta v}$, where $v_{0} \hat{x}_{\|}=\langle\vec{v}\rangle$ is the spontaneous average value of $\vec{v}$ in the ordered phase. We will choose $v_{0}$ $=\sqrt{\alpha / \beta}$ (which should be thought of as an implicit condition on $v_{0}$, since $\alpha$ and $\beta$ can, in general, depend on $|\vec{v}|^{2}$ ); with this choice, the equation of motion for the fluctuation $\delta v_{\|}$of $v_{\|}$is

$$
\partial_{t} \delta v_{\|}=-\sigma_{1} \partial_{\|} \delta \rho-2 \alpha \delta v_{\|}+\text {irrelevant terms }
$$

Note now that if we are interested in "hydrodynamic" modes, by which we mean modes for which frequency $\omega$ $\rightarrow 0$ as wave vector $q \rightarrow 0$, we can, in the hydrodynamic $(\omega, q \rightarrow 0)$ limit, neglect $\partial_{t} \delta v_{\|}$relative to $\alpha \delta v_{\|}$in Eq. (3.1). The resultant equation can trivially be solved for $\delta v_{\|}$:

$$
\delta v_{\|}=-D_{\rho} \partial_{\|} \delta \rho
$$

where we have defined another diffusion constant $D_{\rho}$ $\equiv \sigma_{1} / 2 \alpha$. Inserting Eq. (3.2) in the equations of motion for $\vec{v}_{\perp}$ and $\delta \rho$, we obtain, neglecting "irrelevant" terms:

$$
\begin{aligned}
\partial_{t} \vec{v}_{\perp} & +\gamma \partial_{\|} \vec{v}_{\perp}+\lambda_{1}\left(\vec{v}_{\perp} \cdot \vec{\nabla}_{\perp}\right) \vec{v}_{\perp}+\lambda_{2}\left(\vec{\nabla}_{\perp} \cdot \vec{v}_{\perp}\right) \vec{v}_{\perp} \\
& =-\vec{\nabla}_{\perp} P+D_{B} \vec{\nabla}_{\perp}\left(\vec{\nabla}_{\perp} \cdot \vec{v}_{\perp}\right)+D_{T} \nabla_{\perp}^{2} \vec{v}_{\perp}+D_{\|} \partial_{\|}^{2} \vec{v}_{\perp}+\vec{f}_{\perp},
\end{aligned}
$$

$$
\frac{\partial \delta \rho}{\partial t}+\rho_{o} \vec{\nabla}_{\perp} \cdot \vec{v}_{\perp}+\vec{\nabla}_{\perp} \cdot\left(\vec{v}_{\perp} \delta \rho\right)+v_{0} \partial_{\|} \delta \rho=D_{\rho} \partial_{\|}^{2} \delta \rho
$$

where $D_{\rho}, D_{B}, D_{T}$, and $D_{\|} \equiv D_{T}+D_{2} v_{0}^{2}$ are the diffusion constants, and we have defined 


$$
\gamma \equiv \lambda_{1} v_{0}
$$

The pressure $P$ continues to be given, as it always will, by Eq. (2.7)

From this point forward, we will treat the phenomenological parameters $\lambda_{i}, \gamma$, and $D_{i}$ appearing in Eqs. (3.3) and (3.4) as constants, since they depend, in our original model (2.6), only on the scalar quantities $|\vec{v}|^{2}$ and $\rho(\vec{r})$, whose fluctuations in the broken symmetry state away from their mean values $v_{0}^{2}$ and $\rho_{0}$ are small. Furthermore, these fluctuations lead only to "irrelevant" terms in the equations of motion.

It should be emphasized here that, once nonlinear fluctuation effects are included, the $v_{0}$ in Eq. (3.4) will not be given by the "mean'" velocity of the birds, in the sense of

$$
\langle v\rangle \equiv \frac{\left|\Sigma_{i} \vec{v}_{i}\right|}{N},
$$

where $N$ is the number of birds. This is because, in our continuum language,

$$
\langle v\rangle=\frac{\left|\left\langle\rho \rho(\vec{r}, t) \vec{v}(\vec{r}, t) d^{d} r\right\rangle\right|}{\left\langle\int \rho(\vec{r}, t) d^{d} r\right\rangle}=\frac{|\langle\rho \vec{v}\rangle|}{\langle\rho\rangle}
$$

while $v_{0}$ in Eq. (3.5) is

$$
v_{0}=|\langle\vec{v}(\vec{r}, t)\rangle|
$$

Once $\rho$ fluctuates, so that $\rho=\langle\rho\rangle+\delta \rho$, the "mean" velocity of the birds

$$
\langle v\rangle=\left|\frac{\langle\rho \vec{v}\rangle}{\langle\rho\rangle}\right|=\left|\frac{\langle\rho\rangle\langle\vec{v}\rangle}{\langle\rho\rangle}+\frac{\langle\delta \rho \vec{v}\rangle}{\langle\rho\rangle}\right|,
$$

which only $=v_{0} \equiv|\langle\vec{v}\rangle|$ if the correlation function $\langle\delta \rho \vec{v}\rangle$ $=0$, which it will not, in general. For instance, one could easily imagine that denser regions of the flock might move faster, in which case $\langle\delta \rho \vec{v}\rangle$ would be positive along $\langle\vec{v}\rangle$. Thus, $\langle\vec{v}\rangle$ measured in a simulation by simply averaging the speed of all birds, as in Eq. (3.6), will not be equal to $v_{0}$ in Eq. (3.5). Indeed, we can think of no simple way to measure $v_{0}$, and so chose instead to think of it as an additional phenomenological parameter in the broken symmetry state equations of motion (3.3). It should, in simulations and experiments, be determined by fitting the correlation functions we will calculate in the next section. One should not expect it to be given by $\langle v\rangle$ as defined in Eq. (3.7).

Similar considerations apply to $\gamma$ : it should also be thought of as an independent, phenomenological parameter, not necessarily determined by the mean velocity and nonlinear parameter $\lambda_{1}$ through Eq. (3.5).

\section{LINEARIZED THEORY OF THE BROKEN SYMMETRY STATE}

As a first step towards understanding the implications of these equations of motion, we linearize them in $\vec{v}_{\perp}$ and $\delta \rho$ $\equiv \rho-\rho_{0}$. Doing this, and Fourier transforming in space and time, we obtain the linear equations

$$
\begin{gathered}
{\left[-i\left(\omega-\gamma q_{\|}\right)+\Gamma_{T}(\vec{q})\right] \vec{v}_{T}(\vec{q}, \omega)=\vec{f}_{T}(\vec{q}, \omega),} \\
{\left[-i\left(\omega-\gamma q_{\|}\right)+\Gamma_{L}(\vec{q})\right] v_{L}+i \sigma_{1} q_{\perp} \delta \rho=f_{L}(\vec{q}, \omega),} \\
{\left[-i\left(\omega-v_{0} q_{\|}\right)+\Gamma_{\rho}(\vec{q})\right] \delta \rho+i \rho_{0} q_{\perp} v_{L}=0,}
\end{gathered}
$$

where

$$
v_{L}(\vec{q}, \omega) \equiv \frac{\vec{q}_{\perp} \cdot \vec{v}_{\perp}(\vec{q}, \omega)}{q_{\perp}}
$$

and

$$
\vec{v}_{T}(\vec{q}, \omega)=\vec{v}_{\perp}(\vec{q}, \omega)-\frac{\vec{q}_{\perp} v_{L}}{q_{\perp}}
$$

are the longitudinal and transverse (to $\vec{q}_{\perp}$ ) pieces of the velocity, $\vec{f}_{T}(\vec{q}, \omega)$ and $f_{L}(\vec{q}, \omega)$ are the analogous pieces of the Fourier-transformed random force $\vec{f}(\vec{q}, \omega)$, and we have defined wave-vector-dependent transverse, longitudinal, and $\rho$ dampings $\Gamma_{L, T, \rho}$ :

$$
\begin{gathered}
\Gamma_{L}(\vec{q}) \equiv D_{L} q_{\perp}^{2}+D_{\|} q_{\|}^{2}, \\
\Gamma_{T}(\vec{q})=D_{T} q_{\perp}^{2}+D_{\|} q_{\|}^{2}, \\
\Gamma_{\rho}(\vec{q})=D_{\rho} q_{\|}^{2},
\end{gathered}
$$

where we have defined $D_{L} \equiv D_{T}+D_{B}, q_{\perp}=\left|\vec{q}_{\perp}\right|$.

Note that in $d=2$, the transverse velocity $\vec{v}_{T}$ does not exist: no vector can be perpendicular to both the $x_{\|}$axis and $\vec{q}_{\perp}$ in two dimensions. This leads to many important simplifications in $d=2$, as we will see later; these simplifications make it (barely) possible to get exact exponents in $d=2$ for the full, nonlinear problem.

The normal modes of these equations are $d-2$ purely diffusive transverse modes associated with $\vec{v}_{T}$, all of which have the same eigenfrequency

$$
\omega_{T}=\gamma q_{\|}-i \Gamma_{T}(\vec{q})=\gamma q_{\|}-i\left(D_{T} q_{\perp}^{2}+D_{\|} q_{\|}^{2}\right),
$$

and a pair of damped, propagating sound modes with complex (in both senses of the word) eigenfrequencies

$$
\begin{aligned}
\omega_{ \pm}= & c_{ \pm}\left(\theta_{\vec{q}}\right) q-i \Gamma_{L}\left[\frac{v_{ \pm}\left(\theta_{\vec{q}}\right)}{2 c_{2}\left(\theta_{\vec{q}}\right)}\right]-i \Gamma_{\rho}\left[\frac{v_{\mp}\left(\theta_{\vec{q}}\right)}{2 c_{2}\left(\theta_{\vec{q}}\right)}\right] \\
= & c_{ \pm}\left(\theta_{\vec{q}}\right) q-i\left(D_{L} q_{\|}^{2}+D_{\perp} q_{\perp}^{2}\right)\left[\frac{v_{ \pm}\left(\theta_{q}\right)}{2 c_{2}\left(\theta_{\vec{q}}\right)}\right] \\
& -i D_{\rho} q_{\|}^{2}\left[\frac{v_{\mp}\left(\theta_{\vec{q}}\right)}{2 c_{2}\left(\theta_{\vec{q}}\right)}\right],
\end{aligned}
$$

where $\theta_{\vec{q}}$ is the angle between $\vec{q}$ and the direction of flock motion (i.e., the $x_{\|}$axis), 


$$
\begin{gathered}
c_{ \pm}\left(\theta_{\vec{q}}\right)=\frac{\gamma+v_{0}}{2} \cos \left(\theta_{\vec{q}}\right) \pm c_{2}\left(\theta_{\vec{q}}\right), \\
v_{ \pm}\left(\theta_{\vec{q}}\right)= \pm \frac{\gamma-v_{0}}{2} \cos \left(\theta_{\vec{q}}\right)+c_{2}\left(\theta_{\vec{q}}\right), \\
c_{2}\left(\theta_{\vec{q}}\right) \equiv \sqrt{\frac{1}{4}\left(\gamma-v_{0}\right)^{2} \cos ^{2}\left(\theta_{\vec{q}}\right)+c_{0}^{2} \sin ^{2}\left(\theta_{\vec{q}}\right),}
\end{gathered}
$$

and $c_{0} \equiv \sqrt{\sigma_{1} \rho_{0}}$. A polar plot of this highly anisotropic sound speed is given in Fig. 2. We remind the reader that here and hereafter, we only keep the leading-order terms in the long-wavelength limit, i.e., for small $q_{\|}$and $q_{\perp}$.
The linear equations (4.1)-(4.3) are easily solved for the fields $\delta \rho, \vec{v}_{T}$, and $v_{L}$ in terms of the random forces:

$$
\begin{gathered}
\vec{v}_{T}(\vec{q}, \omega)=G_{T T}(\vec{q}, \omega) \vec{f}_{T}(\vec{q}, \omega), \\
v_{L}(\vec{q}, \omega)=G_{L L}(\vec{q}, \omega) f_{L}(\vec{q}, \omega)+G_{L \rho}(\vec{q}, \omega) f_{\rho}(\vec{q}, \omega), \\
\delta \rho(\vec{q}, \omega)=G_{\rho L}(\vec{q}, \omega) f_{L}(\vec{q}, \omega)+G_{\rho \rho}(\vec{q}, \omega) f_{\rho}(\vec{q}, \omega),
\end{gathered}
$$

where the propagators are

$$
\begin{gathered}
G_{T T}=\frac{1}{-i\left(\omega-\gamma q_{\|}\right)+\Gamma_{T}(\vec{q})}, \\
G_{L L}=\frac{i\left(\omega-v_{0} q_{\|}\right)-\Gamma_{\rho}(\vec{q})}{\left[\omega-c_{+}\left(\theta_{\vec{q}}\right) q\right]\left[\omega-c_{-}\left(\theta_{\vec{q}}\right) q\right]+i \omega\left[\Gamma_{L}(\vec{q})+\Gamma_{\rho}(\vec{q})\right]-i q_{\|}\left[v_{0} \Gamma_{L}(\vec{q})+\gamma \Gamma_{\rho}(\vec{q})\right]}, \\
G_{L \rho}=\frac{i \sigma_{1} q_{\perp}}{\left[\omega-c_{+}\left(\theta_{\vec{q}}\right) q\right]\left[\omega-c_{-}\left(\theta_{\vec{q}}\right) q\right]+i \omega\left[\Gamma_{L}(\vec{q})+\Gamma_{\rho}(\vec{q})\right]-i q_{\|}\left[v_{0} \Gamma_{L}(\vec{q})+\gamma \Gamma_{\rho}(\vec{q})\right]} \\
G_{\rho L}=\frac{i \rho_{0} q_{\perp}}{\left[\omega-c_{+}\left(\theta_{\vec{q}}\right) q\right]\left[\omega-c_{-}\left(\theta_{\vec{q}}\right) q\right]+i \omega\left[\Gamma_{L}(\vec{q})+\Gamma_{\rho}(\vec{q})\right]-i q_{\|}\left[v_{0} \Gamma_{L}(\vec{q})+\gamma \Gamma_{\rho}(\vec{q})\right]}, \\
G_{\rho \rho}=\frac{i\left(\omega-\gamma q_{\|}\right)-\Gamma_{L}(\vec{q})}{\left[\omega-c_{+}\left(\theta_{\vec{q}}\right) q\right]\left[\omega-c_{-}\left(\theta_{\vec{q}}\right) q\right]+i \omega\left[\Gamma_{L}(\vec{q})+\Gamma_{\rho}(\vec{q})\right]-i q_{\|}\left[v_{0} \Gamma_{L}(\vec{q})+\gamma \Gamma_{\rho}(\vec{q})\right]}
\end{gathered}
$$

In writing the definitions of the propagators (4.14)-(4.16), we have introduced a fictitious force $f_{\rho}$ in the $\rho$ equation of motion (4.3). Of course, this force is, in fact, zero; but the propagators $G_{\rho \rho}$ and $G_{L \rho}$ nonetheless prove useful in the perturbative treatment of the nonlinear corrections to this linear theory, so we have included $f_{\rho}$ here.

Given the expressions (4.14)-(4.21) for the velocity and density in terms of the random force $\vec{f}$, and the autocorrelation (2.9) of that random force, it is straightforward to calculate the correlations of the densities and velocities. We find

$$
\begin{aligned}
C_{i j}(\vec{q}, \omega) & \equiv\left\langle v_{i}^{\perp}(-\vec{q},-\omega) v_{j}^{\perp}(\vec{q}, \omega)\right\rangle \\
& =G_{T T}(\vec{q}, \omega) G_{T T}(-\vec{q},-\omega)\left\langle f_{T_{i}}(\vec{q}, \omega) f_{T_{j}}(-\vec{q},-\omega)\right\rangle+G_{L L}(\vec{q}, \omega) G_{L L}(-\vec{q},-\omega) \frac{q_{i}^{\perp} q_{j}^{\perp}}{q_{\perp}^{2}}\left\langle f_{L}(\vec{q}, \omega) f_{L}(-\vec{q},-\omega)\right\rangle \\
& \equiv C_{T T}(\vec{q}, \omega) P_{i j}^{\perp}(\vec{q})+C_{L L}(\vec{q}, \omega) L_{i j}^{\perp}(\vec{q}),
\end{aligned}
$$

where

$$
\begin{gathered}
L_{i j}^{\perp}(\vec{q}) \equiv \frac{q_{i}^{\perp} q_{j}^{\perp}}{q_{\perp}^{2}}, \\
P_{i j}^{\perp}(\vec{q}) \equiv \delta_{i j}^{\perp}-L_{i j}^{\perp}(\vec{q})
\end{gathered}
$$

are longitudinal and transverse projection operators that project any vector perpendicular to both the flock motion and $\vec{q}_{\perp}$,

$$
C_{T T}(\vec{q}, \omega)=\frac{\Delta}{\left(\omega-\gamma q_{\|}\right)^{2}+\Gamma_{T}^{2}(\vec{q})}
$$


and

$$
C_{L L}(\vec{q}, \omega)=\frac{\Delta\left(\omega-v_{0} q_{\|}\right)^{2}}{\left[\omega-c_{+}\left(\theta_{\vec{q}}\right) q\right]^{2}\left[\omega-c_{-}\left(\theta_{\vec{q}}\right) q\right]^{2}+\left\{\omega\left[\Gamma_{L}(\vec{q})+\Gamma_{\rho}(\vec{q})\right]-q_{\|}\left[v_{0} \Gamma_{L}(\vec{q})+\gamma \Gamma_{\rho}(\vec{q})\right]\right\}^{2}}
$$

The transverse and longitudinal correlation functions Eqs. (4.25) and (4.26) are plotted as functions of $\omega$ for fixed $\vec{q}$ in Fig. 6. Note that they have weight in entirely different regions of frequency: $C_{T T}$ is peaked at $\omega=\gamma q_{\|}$, while $C_{L L}$ has two peaks, at $\omega=c_{ \pm}\left(\theta_{\vec{q}}\right) q$. Since all three peaks have widths of order $q^{2}$, there is little overlap between the transverse and the longitudinal peaks as $|\vec{q}| \rightarrow 0$.

The density-density correlation function

$$
C_{\rho \rho}=\frac{\Delta \rho_{0}^{2} q_{\perp}^{2}}{\left[\omega-c_{+}\left(\theta_{\vec{q}}\right) q\right]^{2}\left[\omega-c_{-}\left(\theta_{\vec{q}}\right) q\right]^{2}+\left\{\omega\left[\Gamma_{L}(\vec{q})+\Gamma_{\rho}(\vec{q})\right]-q_{\|}\left[v_{0} \Gamma_{L}(\vec{q})+\gamma \Gamma_{\rho}(\vec{q})\right]\right\}^{2}}
$$

looks almost identical to $C_{L L}$, especially when one notes that near the frequencies $\omega=c_{ \pm}\left(\theta_{\vec{q}}\right) q$ where both peak, the numerator of Eq. (4.26) $\left\{=\left[c_{ \pm}\left(\theta_{\vec{q}}\right) q-v_{0} q_{\|}\right]^{2}\right\}$, differs from that of Eq. (4.27) only by a $|\vec{q}|$-independent factor of $\left[c_{ \pm}\left(\theta_{\vec{q}}\right) q-v_{0} q_{\|}\right]^{2} / \rho_{0} \sigma_{1} q_{\perp}^{2}$.

Given these Fourier-transformed correlation functions, it is straightforward, and instructive, to Fourier transform back to real time. In particular, it is simple to calculate the spatially Fourier-transformed equal-time velocity correlation function:

$$
\begin{aligned}
\left\langle v_{i}(\vec{q}, t) v_{j}(-\vec{q}, t)\right\rangle= & P_{i j}^{\perp}(\vec{q}) \int_{-\infty}^{\infty} \frac{d \omega}{2 \pi} C_{T T}(\vec{q}, \omega) \\
& +L_{i j}^{\perp}(\vec{q}) \int_{-\infty}^{\infty} \frac{d \omega}{2 \pi} C_{L L}(\vec{q}, \omega) \\
= & \frac{\Delta}{2}\left[\frac{P_{i j}^{\perp}(\vec{q})}{\Gamma_{T}(\vec{q})}+\phi(\hat{q}) \frac{L_{i j}^{\perp}(\vec{q})}{\Gamma_{L}(\vec{q})}\right] \propto \frac{1}{q^{2}},
\end{aligned}
$$

where the second integral over frequency has been evaluated in the limit of $|\vec{q}| \rightarrow 0$, so that $c\left(\theta_{\vec{q}}\right) q \gg \Gamma_{L} \propto q^{2}$, and the factor $\phi(\hat{q})$ depends only on the direction $\hat{q}$ of $\vec{q}$, not its magnitude, and is given by the sadly complicated expression

$$
\begin{aligned}
\phi(\hat{q}) \equiv & \frac{1}{c_{2}\left(\theta_{\vec{q}}\right) q}\left[\frac{\left[c_{+}\left(\theta_{\vec{q}}\right) q-v_{0} q_{\|}\right]^{2}}{c_{+}\left(\theta_{\vec{q}}\right) q-v_{0} q_{\|}+\left[c_{+}\left(\theta_{\vec{q}}\right) q-\gamma q_{\|}\right] \Gamma_{\rho} / \Gamma_{L}}\right. \\
& \left.+\frac{\left[c_{-}\left(\theta_{\vec{q}}\right) q-v_{0} q_{\|}\right]^{2}}{c_{-}\left(\theta_{\vec{q}}\right) q-v_{0} q_{\|}+\left[c_{-}\left(\theta_{\vec{q}}\right) q-\gamma q_{\|}\right] \Gamma_{\rho} / \Gamma_{L}}\right] \\
\equiv & \frac{1}{F(\vec{q}, \kappa, \gamma)}\left[\frac{A_{+}^{2}(\vec{q}, \kappa, \gamma)}{A_{+}(\vec{q}, \kappa, \gamma)-A_{-}(\vec{q}, \kappa, \gamma) \Gamma_{\rho}(\vec{q}) / \Gamma_{L}(\vec{q})}\right. \\
& \left.+\frac{A_{-}^{2}(\vec{q}, \kappa, \gamma)}{A_{-}(\vec{q}, \kappa, \gamma)-A_{+}(\vec{q}, \kappa, \gamma) \Gamma_{\rho}(\vec{q}) / \Gamma_{L}(\vec{q})}\right],
\end{aligned}
$$

where we have defined

$$
\begin{aligned}
F(\vec{q} ; \kappa, \gamma) & \equiv \sqrt{\left(\frac{\gamma-v_{0}}{2 v_{0}}\right)^{2} \kappa^{2}\left(\frac{q_{\|}}{q_{\perp}}\right)^{2}+1}, \\
A_{ \pm}(\vec{q} ; \kappa, \gamma) & \equiv \pm F(\vec{q} ; \kappa, \gamma)+\left(\frac{\gamma-v_{0}}{2 v_{0}}\right) \kappa \frac{q_{\|}}{q_{\perp}}
\end{aligned}
$$

and

$$
\kappa \equiv \frac{v_{0}}{\sqrt{\sigma_{1} \rho_{0}}}
$$

The second equality in Eq. (4.29) is obtained from the first simply by canceling common factors of $\sigma_{1} \rho_{0} q_{\perp}^{2}$ out of the numerator and denominator of various terms.

Note, and this will prove to be crucial later, that $\phi(\hat{q})$ depends only on $\hat{q}$, diffusion constants, and the dimensionless ratios $\kappa$ and $\gamma / v_{0}$. This last fact is essential for our renormalization-group scaling analysis, as we will show later.

The $1 / q^{2}$ divergence of Eq. (4.28) as $|\vec{q}| \rightarrow 0$ reflects the enormous long-wavelength fluctuations in this system.

These fluctuations predicted by the linearized theory are strong enough to destroy long-ranged order in $d \leqslant 2$. To see this, calculate the mean-squared fluctuations in $\vec{v}_{\perp}(\vec{r}, t)$ at a given point $\vec{r}$, and time $t$. This is simply the integral of the trace of Eq. (4.28) over all $\vec{q}$ :

$$
\begin{aligned}
\left\langle\left|\vec{v}_{\perp}(\vec{r}, t)\right|^{2}\right\rangle= & \int \frac{d^{d} q}{(2 \pi)^{d}}\left\langle v_{i}(\vec{q}, t) v_{i}(-\vec{q},-t)\right\rangle \\
= & \frac{\Delta}{2}\left[\int \frac { d ^ { d } q } { ( 2 \pi ) ^ { d } } \left(\frac{(d-2)}{D_{T} q_{\perp}^{2}+D_{\|} q_{\|}^{2}}\right.\right. \\
& \left.\left.+\frac{\phi(\hat{q})}{D_{L} q_{\perp}^{2}+D_{\|} q_{\|}^{2}}\right)\right]
\end{aligned}
$$

The last integral clearly diverges in the infrared $(|\vec{q}| \rightarrow 0)$ for $d \leqslant 2$. The divergence in the ultraviolet $(|\vec{q}| \rightarrow \infty)$ for $d \geqslant 2$ is not a concern, since we do not expect our theory to apply for $|\vec{q}|$ larger than the inverse of a microscopic length (such as 


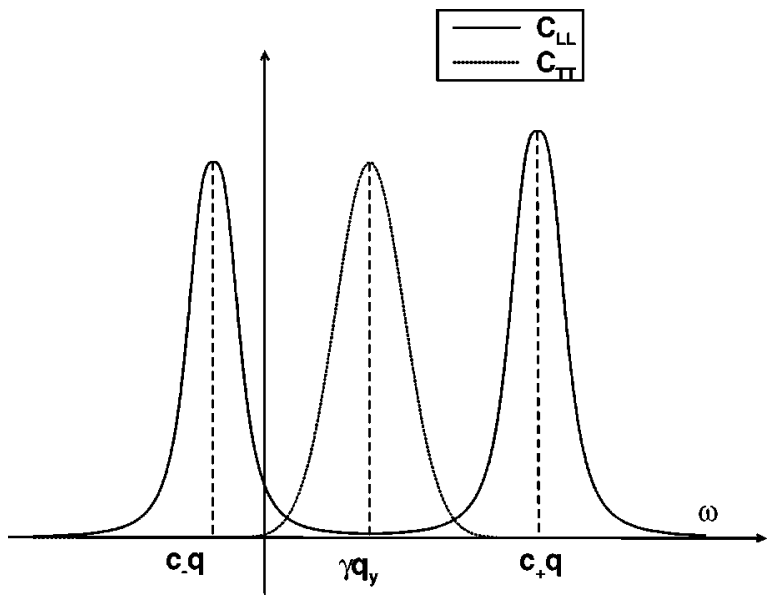

FIG. 6. Plot of $C_{L L}(\vec{q}, \omega)$ and $C_{T T}(\vec{q}, \omega)$ vs $\omega$ for identical fixed $\vec{q}$. Note the smallness of the overlap between the transverse and longitudinal peaks.

the interaction range $\ell_{0}$ ). Presumably, at larger wave numbers, the correlation function falls off fast enough that the wave-vector integral in Eq. (4.33) converges in the ultraviolet.

Indeed, we will in subsequent calculations mimic the effect of this putative more rapid decay of correlations as $|\vec{q}|$ $\rightarrow \infty$ with a sharp ultraviolet cutoff. We will restrict integrals over wave vectors to hypercylindrical shell with long (very long) axis along the direction of flock motion $x_{\|}$:

$$
\left|\vec{q}_{\perp}\right|<\Lambda, \quad-\infty \leqslant q_{\|} \leqslant \infty
$$

with the ultraviolet cutoff $\Lambda$ of order the inverse of a microscopic length (e. g., $\left.\ell_{0}\right)$.

Obviously, this is quite an arbitrary choice of ultraviolet cutoff, and any result that depends on the precise form of this cutoff will not be accurately calculated by this prescription. However, universal, long-wavelength properties of the flock should be unaffected by the precise choice of cutoff, and it is on those properties that we will focus our attention.

The infrared divergence in Eq. (4.33) for $d \leqslant 2$ cannot be dismissed so easily, since our hydrodynamic theory should get better as $|\vec{q}| \rightarrow 0$. Indeed, in the absence of nonlinear effects, this divergence is real, and signifies the destruction of long-ranged order in the linearized model by fluctuations, even for arbitrarily small noise $\Delta$, in spatial dimensions $d$ $\leqslant 2$, and in particular in $d=2$, where the integral in Eq. (4.28) diverges logarithmically in the infrared. This is so since, if $\left\langle\left|\vec{v}_{\perp}\right|^{2}\right\rangle$ is arbitrarily large even for arbitrarily small $\Delta$, our original assumption that $\vec{v}$ can be written as a mean value $\langle\vec{v}\rangle$ plus a small fluctuation $\vec{v}_{\perp}$ is clearly mistaken; indeed, the divergence of $\vec{v}_{\perp}$ suggests that the velocity can swing through all possible directions, implying that $\langle\vec{v}\rangle=0$ for $d \leqslant 2$.

In $d=2$, this result is very reminiscent of the familiar Mermin-Wagner-Hohenberg (MWH) theorem [5], which states that in equilibrium, a spontaneously broken continuous symmetry is impossible in $d=2$ spatial dimensions, precisely because of the type of logarithmic divergence of fluctuations that we have just found here.
In the next section, we will show that this prediction is invalidated by nonlinear effects, and, in fact, much of the scaling of correlation functions and propagators is changed from that predicted by the linearized theory in spatial dimensions $d \leqslant 4$.

\section{NONLINEAR EFFECTS AND BREAKDOWN OF LINEAR HYDRODYNAMICS IN THE BROKEN SYMMETRY STATE}

\section{A. Scaling analysis}

In this section we analyze the effect of the nonlinearities in Eqs. (3.3) and (3.4) on the long length and time behavior of the system, for spatial dimensions $d<4$. We will rescale lengths, time, and the fields $\vec{v}_{\perp}$ and $\delta \rho$ according to

$$
\begin{gathered}
\vec{x}_{\perp} \rightarrow b \vec{x}_{\perp}, \quad x_{\|} \rightarrow b^{\zeta} x_{\|}, \quad t \rightarrow b^{z} t, \\
\vec{v}_{\perp} \rightarrow b^{\chi} \vec{v}_{\perp}, \quad \delta \rho \rightarrow b^{\chi} \rho \rho,
\end{gathered}
$$

choosing the scaling exponents to keep the diffusion constants $D_{B, T, \rho, \|}$, and the strength $\Delta$ of the noise fixed. The reason for choosing to keep these particular parameters fixed rather than, e.g., $\sigma_{1}$, is that these parameters completely determine the size of the equal time fluctuations in the linearized theory, as can be seen from Eq. (4.33). Under the rescalings (5.1), the diffusion constants rescale according to $D_{B, T} \rightarrow b^{z-2} D_{B, T}$ and $D_{\rho, \|} \rightarrow b^{z-2 \zeta} D_{\rho, \|}$; hence, to keep them fixed, we must choose $z=2$ and $\zeta=1$. The rescaling of the random force $\vec{f}$ can then be obtained from the form of the $f-f$ correlations Eq. (2.9) and is, for this choice of $z$ and $\zeta$,

$$
\vec{f} \rightarrow b^{-1-d / 2} \vec{f} .
$$

To maintain the balance between $\vec{f}$ and the linear terms in $\vec{v}_{\perp}$ in Eq. (3.3), we must rescale the velocity field according to

$$
\vec{v}_{\perp} \rightarrow b^{x^{v_{\perp}}}
$$

with

$$
\chi=1-d / 2,
$$

which is the roughness exponent for the linearized model. That is, we expect $\vec{v}_{\perp}$ fluctuations on length scale $L$ to scale like $L^{\chi}$. Therefore, the linearized hydrodynamic equations, neglecting the nonlinear convective term and the nonlinearities in the pressure, imply that $\vec{v}_{\perp}$ fluctuations grow without bound (like $L^{\chi}$ ) as $L \rightarrow \infty$ for $d \leqslant 2$, where the above expression for $\chi$ becomes positive. Thus, this linearized theory predicts the loss of long-range order in $d \leqslant 2$, as we saw in Sec. IV by explicitly evaluating the real space fluctuations.

Making the rescalings as described in Eqs. (5.1), the equation of motion (3.3) becomes

$$
\begin{aligned}
\partial_{t} \vec{v}_{\perp}+b^{\gamma_{v}} \gamma \partial_{\|} \vec{v}_{\perp}+b^{\gamma_{\lambda}}\left[\lambda_{1}\left(\vec{v}_{\perp} \cdot \vec{\nabla}_{\perp}\right) \vec{v}_{\perp}+\lambda_{2}\left(\vec{\nabla}_{\perp} \cdot \vec{v}_{\perp}\right) \vec{v}_{\perp}\right] \\
=-\vec{\nabla}_{\perp}\left(\sum_{n=1}^{\infty} b^{\gamma_{n}} \sigma_{n}(\delta \rho)^{n}\right)+D_{B} \vec{\nabla}_{\perp}\left(\vec{\nabla}_{\perp} \cdot \vec{v}_{\perp}\right) \\
+D_{T} \nabla_{\perp}^{2} \vec{v}_{\perp}+D_{\|} \partial_{\|} \vec{v}_{\perp}+\vec{f}_{\perp}
\end{aligned}
$$


with

$$
\begin{gathered}
\gamma_{\lambda}=\chi+1=2-d / 2, \\
\gamma_{v}=z-\zeta=1,
\end{gathered}
$$

and

$$
\gamma_{n}=z-\chi+n \chi-1=n+(1-n) \frac{d}{2}
$$

The scaling exponent $\chi_{\rho}$ for $\delta \rho$ is given by $\chi_{\rho}=\chi$, since the density fluctuations $\delta \rho$ are comparable in magnitude to the $\vec{v}_{\perp}$ fluctuations. To see this, note that the eigenmode of the linearized equations of motion that involves $\delta \rho$ is a sound mode, with dispersion relation $\omega=c_{ \pm}\left(\theta_{q}\right) q$. Inserting this into the Fourier transform of the continuity equation (3.4), we see that $\delta \rho \sim \vec{q}_{\perp} \cdot \vec{v}_{\perp} / q_{\perp}$. The magnitude of $\vec{q}_{\perp}$ drops out of the right-hand side of this expression; hence $\delta \rho$ scales like $\left|\vec{v}_{\perp}\right|$ at long distances. Therefore, we will choose $\chi_{\rho}=\chi=$ $1-d / 2$.

The first two of these scaling exponents for the nonlinearities to become positive as the spatial dimension $d$ is decreased are $\gamma_{\lambda}$ and $\gamma_{2}$, which both become positive for $d$ $<4$, indicating that the $\lambda_{1}\left(\vec{v}_{\perp} \cdot \vec{\nabla}\right) \vec{v}_{\perp}, \lambda_{2}\left(\vec{\nabla}_{\perp} \cdot \vec{v}_{\perp}\right) \vec{v}_{\perp}$, and $\sigma_{2} \vec{\nabla}_{\perp}\left(\delta \rho^{2}\right)$ nonlinearities are all relevant perturbations for $d<4$. So, for $d<4$, the linearized hydrodynamics will break down.

What can we say about the behavior of Eqs. (3.3) and (3.4) for $d<4$, when the linearized hydrodynamics no longer holds? The standard approach for such problems is the dynamical renormalization group. In most cases, this approach is only practical near the upper critical dimension (in our case $d_{c}=4$ ), and yields the anomalous exponents in an expansion in $\epsilon=d_{c}-d$. This approach will obviously not be of much use in our problem in $d=2$, where the ostensibly small parameter in this expansion $\epsilon=2$. We will nonetheless undertake this approach in Sec. V B, and show that, for unfortunate technical reasons, we learn little even near $d=4$. Fortunately, as we show in Sec. V C, because of the various symmetries in Eq. (3.3), we can obtain the exact scaling exponents in $d=2$.

\section{B. Renormalization-group analysis, $d<4$}

In this subsection, we analyze the effect of the relevant nonlinearities $\lambda_{1}, \lambda_{2}$, and $\sigma_{2}$ on the broken symmetry state in spatial dimensions $d<4$.

Our tool is the dynamical renormalization group (for details, see, e.g., the excellent description in Forster, Nelson, and Stephen [6]). We will summarize the essential features of this procedure here; readers interested in details are referred to [6].

We proceed through the iteration of the following 3 steps:

(1) We separate the fields $\vec{v}_{\perp}$ and $\delta \rho$, and the random forces $\vec{f}$ into short and long wavelength components, according to

$$
\begin{aligned}
& \vec{v}_{\perp>}(\vec{r}, t)=\int_{>} \frac{d^{d} q d \omega}{(2 \pi)^{d}} \vec{v}_{\perp}(\vec{q}, \omega) e^{i(\vec{q} \cdot \vec{r}-\omega t)}, \\
& \vec{v}_{\perp<}(\vec{r}, t)=\int_{<} \frac{d^{d} q d \omega}{(2 \pi)^{d}} \vec{v}_{\perp}(\vec{q}, \omega) e^{i(\vec{q} \cdot \vec{r}-\omega t)},
\end{aligned}
$$

where $\int_{>}$denotes a wave-vector integral restricted to a hypercylindrical shell $b^{-1} \Lambda<\left|\vec{q}_{\perp}\right|<\Lambda$, where $\Lambda$ is an ultraviolet cutoff, and $\int_{<}$likewise denotes an integral over the interior of this shell: $\left|\vec{q}_{\perp}\right|<b^{-1} \Lambda . \quad \delta \rho$ and $\vec{f}_{\perp}$ are likewise separated.

(2) Average the EOM over the short wavelength fields $\vec{v}_{\perp>}, \delta \rho_{>}$, and $\vec{f}_{>}$to get new, effective EOM for the longwavelength fields $\vec{v}_{\perp<}$ and $\delta \rho_{<}$, with "intermediate" renormalized parameters $D_{\perp}^{I}$, etc. This average is performed perturbatively in the nonlinearities in the EOM. The perturbation theory can be represented graphically; the interested reader is referred to the previously mentioned [6] for further details on the mechanics of this.

(3) We now rescale the time, space, and the fields in the EOM according to Eq. (5.1) in order to restore the original ultraviolet cutoff $\Lambda$ of the problem. We will choose rescaling exponents $z, \zeta$, and $\chi$ to produce fixed points.

Of course, the exponents are, in fact, completely arbitrary. We need not choose them to produce fixed points. However, it is very convenient to do so, since, as we will show in more detail later, the values of $z, \zeta$, and $\chi$ that $d o$ produce fixed points are exactly the values of the physical observable time, anisotropy, and roughness exponents that characterize the scaling properties of various correlation functions.

Performing this RG procedure, we find the following recursion relations:

$$
\begin{gathered}
\frac{d D_{B, T}}{d \ell}=\left[z-2+G_{B, T}^{D}(g)\right] D_{B, T}, \\
\frac{d D_{\|, \rho}}{d \ell}=\left[z-2 \zeta+G_{\|, \rho}\left(\left\{g_{i}\right\}\right)\right] D_{\|, \rho}, \\
\frac{d \sigma_{n}}{d \ell}=\left[z+(n-1) \chi-1+G_{n}^{\sigma}\left(\left\{g_{i}\right\}\right)\right] \sigma_{n}, \\
\frac{d \rho_{0}}{d \ell}=(z-1) \rho_{0}, \\
\frac{d \lambda_{1,2, \rho}}{d \ell}=\left[\chi-1+z+G_{1,2, \rho}^{\lambda}\left(\left\{g_{i}\right\}\right)\right] \lambda_{1,2, \rho}, \\
\frac{d \Delta}{d \ell}=\left[z-\zeta-2 \chi+1-d+G_{\Delta}\left(\left\{g_{i}\right\}\right)\right] \Delta, \\
\left.\frac{d \gamma}{d \ell}=(z-\zeta) \gamma, \zeta\right) v_{0}, \\
\frac{d \ell}{d \ell},
\end{gathered}
$$


where we have taken $b=1+d \ell, d \ell \ll 1$, to obtain differential recursion relations, the $G$ 's represent graphical (i.e., perturbative) corrections, and the $\left\{g_{i}\right\}$ 's are a set of dimensionless coupling constants involving ratios of powers of the dynamical parameters. We have also dropped "irrelevant" terms in these recursion relations. The coupling constant $\lambda_{\rho}$ is the coefficient of the $\vec{\nabla}_{\perp} \cdot\left(\vec{v}_{\perp} \delta \rho\right)$ nonlinearity in the $\rho$ equation of motion. This coupling constant is equal to 1 , and must, up to trivial rescaling corrections, remain equal to 1 upon renormalization. This is a simple consequence of the fact that mass conservation is exact; that is, the equation of motion for $\rho$ must remain the simple continuity equation $\partial_{t} \rho+\vec{\nabla} \cdot(\rho \vec{v})=0$, except for the trivial changes introduced by rescaling. This implies that $G_{\rho}^{\lambda}\left(\left\{g_{i}\right\}\right)=0$, exactly, for all $\left\{g_{i}\right\}$. We will later use this fact to obtain exact values for the scaling exponents $\chi, z$, and $\zeta$ in $d=2$. It is worth noting that $\gamma$ is treated as an independent variable here, only its bare value is related to the bare values of $\lambda_{1}$ and $v_{0}$ through Eq. (3.5).

Although there is no symmetry argument forbidding renormalization of $v_{0}$ and $\gamma$, simple power counting shows that there are no relevant graphical corrections to them; this is why no graphical corrections appear in Eqs. (5.17), (5.18).

As mentioned earlier, the rescaling exponents $\chi, z$, and $\zeta$ are arbitrary. We chose them to produce fixed points only for computational convenience. However, there is no choice of rescaling exponents that will keep all the parameters fixed. For instance, to keep $D_{B, T}$ and $D_{\|, \rho}$ fixed, we will have to choose $z>1$. However, with this choice of $z$, the relations (5.13) and (5.14) show that $\sigma_{1}$ and $\rho_{0}$ flow to infinity.

Which of the parameters, then, should we choose to keep fixed? That is, which is most convenient to keep fixed? The answer to this question is provided by the renormalization group matching formalism. This approach enables one to use the renormalization group to relate correlation functions in the original, unrenormalized model at long distances and large times to the same correlation functions in the renormalized system at shorter distances and times. The advantage of this approach is that long distance, large time correlation functions are hard to calculate in $d<4$, since, as we showed from our earlier scaling arguments [and can also verify from the renormalization-group recursion relations (5.11)-(5.12)], these are not accurately calculable from the harmonic theory developed in Sec. IV, since the nonlinearities $\lambda_{1,2}, \lambda_{\rho}$, and $\sigma_{2}$ have very large effects at long distances. By mapping these correlation functions onto those at short distances in the renormalized equations of motion, we circumvent this problem. Clearly, there is a caveat here: even at short distances, the correlation functions in the renormalized model can only be calculated accurately in the harmonic theory if the nonlinear couplings in that renormalized model are not too big. This suggests that the convenient choice of the rescaling exponents $\chi, z$, and $\zeta$ is that which keeps the nonlinearities $\lambda_{1,2}, \lambda_{\rho}$, and $\sigma_{2}$ fixed.

Let us illustrate these considerations explicitly for one very important correlation function: the equal time spatially Fourier-transformed velocity-velocity autocorrelation function:

$$
C_{i j}(\vec{q}) \equiv\left\langle v_{i}(\vec{q}, t) v_{j}(-\vec{q}, t)\right\rangle
$$

This particular correlation function is important because it gives us our best measure of the size of the velocity fluctuations, and will ultimately determine whether or not these fluctuations destroy the long-ranged orientational order of the flock (thereby driving its mean velocity to zero).

$C_{i j}(\vec{q})$ is, of course, a function of the flock dynamical parameters $D_{B, T}, D_{\|, \rho}, \Delta$, etc., as well as of $\vec{q}$. Furthermore, at small $\vec{q}$, it is difficult to calculate in spatial dimension $d<4$ due to the nonlinear terms, for the reasons discussed above. So let us follow this renormalization-group matching procedure to relate $C_{i j}\left(\vec{q} ;\left\{B_{i}^{0}\right\}\right)$ where $\left\{B_{i}^{0}\right\}$ denotes the set of dynamical parameters $D_{B, T}^{0}, D_{\|}^{0}, \Delta_{0}$, etc. in the unrenormalized model, to the same correlation function in the renormalized model, a renormalization group time $\ell$ later:

$$
\begin{aligned}
C_{i j}( & \left.\vec{q}_{\perp}, q_{\|} ;\left\{B_{i}^{0}\right\}\right) \\
\quad & =e^{(2 \chi+\zeta+d-1) \ell} C_{i j}\left[e^{\ell} \vec{q}_{\perp}, e^{\zeta \ell} q_{\|} ;\left\{B_{i}(\ell)\right\}\right],
\end{aligned}
$$

where the $\left\{B_{i}(\ell)\right\}$ denote the renormalized parameters.

In the discussion that follows, we will first consider the case $\left(q_{\|} / \Lambda\right) \ll\left(q_{\perp} / \Lambda\right)^{\zeta}$. At the conclusion of the discussion of this special case, we will briefly indicate how the general case can be treated to obtain the scaling laws quoted in the Introduction. For the case $\left(q_{\|} / \Lambda\right) \ll\left(q_{\perp} / \Lambda\right)^{\zeta}$, we will choose $\ell=\ell_{*}\left(\vec{q}_{\perp}\right)=\ln \left(\Lambda / q_{\perp}\right)$, where $\Lambda$ is the ultraviolet cutoff, on the right-hand side, and obtain

$$
\begin{aligned}
C_{i j}\left(\vec{q}_{\perp}, q_{\|} ;\left\{B_{i}^{0}\right\}\right) \\
\quad=\left(\frac{\Lambda}{q_{\perp}}\right)^{2 \chi+\zeta+d-1} C_{i j}\left(\Lambda, \frac{q_{\|}}{\left(q_{\perp} / \Lambda\right)^{\xi}} ;\left\{B_{i}\left[\ell_{*}\left(\vec{q}_{\perp}\right)\right]\right\}\right) .
\end{aligned}
$$

Now, if the original $q_{\perp}$ was small $(\ll \Lambda)$ and we have chosen the rescaling exponents $\chi, \zeta$, and $z$ so that the nonlinearities $\lambda_{1}(\ell), \lambda_{2}(\ell), \lambda_{\rho}(\ell)$, and $\sigma_{2}(\ell)$ on the right-hand side of Eq. (5.21) flow, as $\ell \rightarrow \infty$, to $O(1)$ fixed point values $\left(\lambda_{1,2, \rho}^{*}, \sigma_{2}^{*}\right)$, then $\lambda_{1}\left(\ell_{*}\right), \lambda_{2}\left(\ell_{*}\right), \lambda_{\rho}\left(\ell_{*}\right)$, and $\sigma_{2}\left(\ell_{*}\right)$ can be replaced by those fixed point values, since $\ell_{*}$ will be large. Because those fixed point values are, by assumption, $O(1)$, then, up to $O(1)$ correction factors coming from these nonlinearities, the right-hand side of Eq. (5.21) can be evaluated in the harmonic approximation Eqs. (4.22), (4.25), and (4.26). [The correction factors are only of $O(1)$-i.e., not divergent-because the right-hand side of Eq. (5.21) is evaluated at large $\vec{q}\left(\left|\vec{q}_{\perp}\right|=\Lambda\right)$, where the infrared divergences associated with the strong relevance of the nonlinearities do not matter. It is precisely because of those infrared divergences that we could not evaluate the left-hand side of (5.21) directly, but rather were forced to go through this seemingly circuitous $R G$ matching formalism.] Making that harmonic approximation on the right-hand side, we obtain 


$$
\begin{aligned}
C_{i j}\left(\Lambda, \frac{q_{\|}}{\left(q_{\perp} / \Lambda\right)^{\zeta}} ;\left\{\Gamma\left(\ell_{*}\right)\right\}\right) \\
=\frac{\Delta_{*}}{D_{T}^{*} \Lambda^{2}+D_{\|}^{*}\left[q_{\|} /\left(q_{\perp} / \Lambda\right)^{\zeta}\right]^{2}} P_{i j}^{\perp}\left(\hat{q}_{\perp}\right) \\
\quad+\frac{\Delta_{*}}{D_{L}^{*} \Lambda^{2}+D_{\|}^{*}\left[q_{\|} /\left(q_{\perp} / \Lambda\right)^{\zeta}\right]^{2}} \\
\quad \times \phi\left(\Lambda, \frac{q_{\|}}{\left(q_{\perp} / \Lambda\right)^{\zeta}} ;\left\{B_{i}\left(\ell_{*}\right)\right\}\right) L_{i j}^{\perp}\left(\hat{q}_{\perp}\right),
\end{aligned}
$$

where we have used the fact that we have chosen the scaling exponents to make $\Delta$ and all the diffusion coefficients $\left\{D_{i}\right\}$ flow to fixed points $\Delta^{*},\left\{D_{i}^{*}\right\}$. We wish to show that this expression depends on $\vec{q}$ only through the scaling ratio $u$ $\equiv q_{\|} /\left(q_{\perp} / \Lambda\right)^{\zeta}$. The first (transverse) term in Eq. (5.22) explicitly has this property. The second (longitudinal term) would also, except for the $\phi$ factor, to which we now turn. From Eq. (4.29) for $\phi$, we see that to calculate this factor we must calculate

$$
\begin{aligned}
F\left(\Lambda, \frac{q_{\|}}{\left(q_{\perp} / \Lambda\right)^{\zeta}} ;\left\{B_{i}\left(\ell_{*}\right)\right\}\right) \\
\quad=\sqrt{\left(\frac{\gamma\left(\ell_{*}\right)-v_{0}\left(\ell_{*}\right)}{2 v_{0}\left(\ell_{*}\right)}\right)^{2} \kappa^{2}\left(\ell_{*}\right)\left(\frac{q_{\|}}{\Lambda\left(q_{\perp} / \Lambda\right)^{\zeta}}\right)^{2}+1},
\end{aligned}
$$

$$
\begin{aligned}
& A_{ \pm}\left(\Lambda, \frac{q_{\|}}{\left(q_{\perp} / \Lambda\right)^{\zeta}} ;\left\{B_{i}\left(\ell_{*}\right)\right\}\right) \\
&= \pm F\left(\Lambda, \frac{q_{\|}}{\left(q_{\perp} / \Lambda\right)^{\zeta}} ;\left\{B_{i}\left(\ell_{*}\right)\right\}\right) \\
&-\frac{\left[\gamma\left(\ell_{*}\right)-v_{0}\left(\ell_{*}\right)\right]}{2 v_{0}\left(\ell_{*}\right)} \kappa\left(\ell_{*}\right)\left(\frac{q_{\|}}{\Lambda\left(q_{\perp} / \Lambda\right)^{\zeta}}\right)
\end{aligned}
$$

and

$$
\begin{aligned}
B_{ \pm}\left(\Lambda, \frac{q_{\|}}{\left(q_{\perp} / \Lambda\right)^{\zeta}} ;\left\{B_{i}\left(\ell_{*}\right)\right\}\right) \\
= \pm F\left(\Lambda, \frac{q_{\|}}{\left(q_{\perp} / \Lambda\right)^{\zeta}} ;\left\{B_{i}\left(\ell_{*}\right)\right\}\right) \\
\quad+\frac{\left[\gamma\left(\ell_{*}\right)-v_{0}\left(\ell_{*}\right)\right]}{2 v_{0}\left(\ell_{*}\right)} \kappa\left(\ell_{*}\right)\left(\frac{q_{\|}}{\Lambda\left(q_{\perp} / \Lambda\right)^{\zeta}}\right),
\end{aligned}
$$

all of which are clearly dependent only on the fixed point value of the ratio $\gamma\left(\ell_{*}\right) / v_{0}\left(\ell_{*}\right)$ (which is just a number of $O(1)$ since $\gamma\left(\ell_{*}\right)$ and $v_{0}\left(\ell_{*}\right)$ have the same dependence on $\ell_{*}: \exp \left[(z-\zeta) \ell_{*}\right]$, as can be seen from their recursion relations), and the combination

$$
\kappa\left(\ell_{*}\right) \frac{q_{\|}}{\left(q_{\perp} / \Lambda\right)^{\zeta} \Lambda} .
$$

By combining the recursion relations (5.17), (5.13), and (5.14) into a recursion relation for $\kappa$ :

$$
\frac{d}{d \ell}(\ln \kappa)=\frac{d \ln v_{0}}{d \ell}-\frac{1}{2} \frac{d}{d \ell}\left(\ln \sigma_{1}+\ln \rho_{0}\right)=1-\zeta
$$

we find

$$
\kappa(\ell)=e^{(1-\zeta) \ell} \kappa_{0},
$$

which implies that

$$
\kappa\left(\ell_{*}\right)=\kappa_{0}\left(e^{\ell} *\right)^{1-\zeta}=\kappa_{0}\left(\frac{\Lambda}{q_{\perp}}\right)^{1-\zeta}
$$

Using this in Eq. (5.26), we see that the combination

$$
\kappa\left(\ell_{*}\right) \frac{q_{\|}}{\Lambda\left(q_{\perp} / \Lambda\right)^{\zeta}}=\kappa_{0} \frac{q_{\|}}{q_{\perp}}
$$

takes on precisely the value it would take on using the unrenormalized parameters and the unrescaled wave vector $\vec{q}$. Hence, the same is true of $F, A_{ \pm}$, and $B_{ \pm}$. And, therefore, the same is true of $\phi(\hat{q})$.

Thus, we can replace $\phi\left(\Lambda, q_{\|} /\left(q_{\perp} / \Lambda\right)^{\zeta} ;\left\{B_{i}\left(\ell_{*}\right)\right\}\right)$ in Eq. (5.22) with $\phi\left(\hat{q} ;\left\{B_{i}^{0}\right\}\right)$, its unrenormalized value straight from the linearized theory. Doing so, and recalling that the unrenormalized $\phi(\hat{q})$ was $O(1)$ for all directions $\hat{q}$ of $\vec{q}$, we see from Eq. (5.22) that the correlation function $C_{i j}$ is largest when

$$
\frac{q_{\|}}{\Lambda} \sim\left(\frac{q_{\perp}}{\Lambda}\right)^{\zeta}
$$

For $|\vec{q}| \ll \Lambda$, where our theory applies, Eq. (5.31) implies that $q_{\|} \gg q_{\perp}$ (since $\left.\zeta<1\right)$. In that limit, $\phi(\hat{q}) \rightarrow 1$; using this in the expression (5.22) for $C_{i j}$ in the renormalized system, and using Eq. (5.22) in turn in our expression (5.21) for $C_{i j}$ in the original model, we obtain, for $q_{\|} \gg q_{\perp}$, the scaling law

$$
C_{i j}(\vec{q})=q_{\perp}^{-(2 \chi+d+\zeta-1)} f_{i j}\left(\frac{\left(q_{\|} / \Lambda\right)}{\left(q_{\perp} / \Lambda\right)^{\zeta}}\right)
$$

Note that the range $q_{\|} \gg q_{\perp}$ for which this scaling law holds includes those $\vec{q}$ 's which dominate the fluctations, namely, those with $q_{\|} / \Lambda \gtrsim\left(q_{\perp} / \Lambda\right)^{\zeta}$.

Integrating $C_{i j}(\vec{q})$ over all $\vec{q}$ gives the equal-time, rootmean-squared real-space fluctuation of $\vec{v}_{\perp}$ : 


$$
\begin{aligned}
\left\langle\left|\vec{v}_{\perp}(\vec{r}, t)\right|^{2}\right\rangle & =\int \frac{d^{d} q}{(2 \pi)^{d}} C_{i i}(\vec{q}) \\
& =\int \frac{d^{d-1} q_{\perp}}{(2 \pi)^{d}} q_{\perp}^{-(2 \chi+d+\zeta-1)} \int d q_{\|} f_{i i}\left(\frac{\left(q_{\|} / \Lambda\right)}{\left(q_{\perp} / \Lambda\right)^{\zeta}}\right) \\
& =A \int \frac{d^{d-1} q_{\perp}}{q_{\perp}^{2 \chi^{+} d-1}},
\end{aligned}
$$

where the final proportionality was obtained by scaling $q_{\perp}$ out of the $q_{\|}$integral via the change of variables $q_{\|}$ $\equiv \Lambda Q_{\|}\left(q_{\perp} / \Lambda\right)^{\zeta}$ and we have defined the $q_{\perp}$-independent

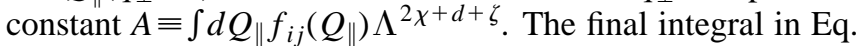
(5.33) clearly converges in the infrared $\left(\left|\vec{q}_{\perp}\right| \rightarrow 0\right)$ limit if and only if $\chi<0$. Furthermore, if $\chi$ is $>0$, and we impose an infrared cutoff $\left|\vec{q}_{\perp}\right|>L_{\perp}^{-1}$ in Eq. (5.33), where $L_{\perp}$ is the lateral (i.e., $\perp$ direction) spatial extent of the system, we easily obtain

$$
\left\langle\left|\vec{v}_{\perp}(\vec{v}, t)\right|^{2}\right\rangle=C^{\prime} L_{\perp}^{2 \chi} .
$$

Indeed, the connected real-space, equal-time, velocity autocorrelation function discussed in the Introduction is given by

$$
\begin{aligned}
C_{C}(\vec{R}) & \equiv\langle\vec{v}(\vec{r}+\vec{R}, t) \cdot \vec{v}(\vec{r}, t)\rangle-|\langle\vec{v}(\vec{r}, t)\rangle|^{2} \\
& =\left\langle\overrightarrow{v_{\perp}}(\vec{r}+\vec{R}, t) \cdot \overrightarrow{v_{\perp}}(\vec{r}, t)\right\rangle \\
& =\int \frac{d^{d} q}{(2 \pi)^{d}} C_{i i}(\vec{q}) e^{i \vec{q} \cdot \vec{R}} \\
= & \int \frac{d^{d} q}{(2 \pi)^{d}} q_{\perp}^{-(2 \chi+d+\zeta-1)} f_{i i}\left(\frac{\left(q_{\|} / \Lambda\right)}{\left(q_{\perp} / \Lambda\right)^{\zeta}}\right) \\
& \times e^{i\left(\vec{q}_{\perp} \cdot \vec{R}_{\perp}+q \| R_{\|}\right)} .
\end{aligned}
$$

Making the changes of variable

$$
\vec{q} \| \frac{\vec{Q}_{\perp}}{\left|\vec{R}_{\perp}\right|}, \quad q_{\perp} \equiv \frac{Q_{\|}}{\left|\vec{R}_{\perp}\right|^{\zeta}},
$$

we obtain the scaling law (1.8) for $C_{c}(\vec{R})$ quoted in the Introduction, with

$$
\begin{aligned}
f_{v}(u) \equiv & \int d^{d-1} Q_{\perp} d Q_{\|} f_{i i}\left(\frac{Q_{\|}}{Q_{\perp}^{\zeta}}\right) \\
& \times e^{i\left(\vec{Q}_{\perp} \cdot \hat{R}_{\perp}+Q_{\|} u\right)} Q_{\perp}^{-(2 \chi+d+\zeta-1)} .
\end{aligned}
$$

This shows that the $\chi$ we obtain from the renormalization group by the prescription we have chosen-namely, making the specific set of parameters $D_{B, T, \|}, \Delta, \lambda_{1,2, \rho}$, and $\sigma_{2}$ flow to fixed points-is precisely the physical roughness exponent defined by the velocity fluctuations.

To summarize: if we chose the rescaling exponents $\chi, z$, and $\zeta$ so as to make the particular subset of the dynamical parameters $D_{B, T, \|, \rho}, \Delta, \lambda_{1,2, \rho}$, and $\sigma_{2}$ flow to nonzero fixed points, then those $\chi$ and $\zeta$ are the ones that appear in the scaling law (1.8). This directly and simply relates the RG to physically observable correlation functions, so this is the choice we will make.

A scaling law similar to Eq. (5.32) can be derived, by precisely the same type of arguments, for the equal-time density-density correlation function:

$$
C_{\rho}(\vec{q})=\frac{q_{\perp}^{3-d-\zeta-2 \chi}}{q^{2}} f_{\rho}\left(\frac{q_{\|} \ell_{0}}{\left(q_{\perp} \ell_{0}\right)^{\zeta}}\right) Y\left(\theta_{q}^{\vec{q}}\right),
$$

where, in writing this relation, we have used the fact that the "roughness" exponent for $\rho, \chi_{\rho}=\chi$, the "roughness" exponent for $\vec{v}_{\perp}$.

The alert reader will have noticed that neither of the scaling laws (5.32) and (5.38) derived so far involves the time rescaling exponent $z$. This is unsurprising, since we have considered only equal-time correlation functions up to now.

To fully study the dynamics of the model, we need to consider correlations between different times, as well as positions. These different time correlation functions will involve $z$.

It is easiest to work with the space and time Fourier transform $C_{i j}(\vec{q}, \omega)$, defined by

$$
\left\langle v_{i}(\vec{q}, \omega) v_{j}\left(-\vec{q}, \omega^{\prime}\right)\right\rangle \equiv \delta\left(\omega+\omega^{\prime}\right) C_{i j}(\vec{q}, \omega) .
$$

We will find, as we have asserted many times already in this paper, that $C_{i j}(\vec{q}, \omega)$ does not have a simple scaling form, unlike the equal-time correlations. Nonetheless we can derive an expression for it in terms of functions of $\vec{q}$ that $d o$ show simple scaling behavior, namely, effective wavevector-dependent diffusion constants that diverge as $|\vec{q}|, \omega$ $\rightarrow 0$

We begin this derivation by separating $C_{i j}$ into its transverse and longitudinal parts:

$$
C_{i j}(\vec{q}, \omega) \equiv L_{i j}^{\perp} C_{L}(\vec{q}, \omega)+P_{i j}^{\perp} C_{T}(\vec{q}, \omega),
$$

where $L_{i j}^{\perp} \equiv q_{i}^{\perp} q_{j}^{\perp} / q_{\perp}^{2}$ and $P_{i j}^{\perp}=\delta_{i j}-L_{i j}^{\perp}-\delta_{i, \|} \delta_{j, \|}$ are, respectively, the longitudinal and transverse projection operators defined in Sec. IV. Both the transverse and longitudinal pieces $C_{L, T}(\vec{q}, \omega)$ obey the same renormalization-group transformation

$$
\begin{aligned}
& C_{L, T}\left(\vec{q}_{\perp}, q_{\|}, \omega ;\left\{B_{i}^{0}\right\}\right) \\
& \quad=e^{\left(2 \chi^{+} z+\zeta+d-1\right) \ell} C_{L, T}\left(e^{\ell} \vec{q}_{\perp}, e^{\zeta \ell} q_{\|}, e^{z \ell} \omega ;\left\{B_{i}(\ell)\right\}\right),
\end{aligned}
$$

where we have been careful to take into account the rescaling of the delta function in Eq. (5.39) in deriving the argument of the exponential in Eq. (5.41).

As for the equal-time correlation function, it is convenient here to choose the rescaling factor $e^{\ell}$ such that $e^{\ell} \vec{q}_{\perp}$ is right on the Brillouin zone boundary; i.e., $e^{\prime}\left|\vec{q}_{\perp}\right|=\Lambda$. Making this choice, and taking $\Lambda=1$, we obtain 


$$
\begin{aligned}
& C_{L, T}\left(\vec{q}_{\perp}, q_{\|}, \omega ;\left\{B_{i}^{0}\right\}\right) \\
& \quad=q_{\perp}^{-(2 \chi+z+\zeta+d-1)} C_{L, T}\left(\hat{q}_{\perp}, \frac{q_{\|}}{q_{\perp}^{\zeta}}, \frac{\omega}{q_{\perp}^{z}} ;\left\{B_{i}\left(\ell_{*}\right)\right\}\right),
\end{aligned}
$$

where, on the right-hand side, we have defined

$$
\ell_{*}=\ln \left(\frac{1}{\left|\vec{q}_{\perp}\right|}\right)
$$

For the moment, let us focus on the longitudinal piece $C_{L}(\vec{q}, \omega)$. As we argued for the equal-time correlation function, here too we can evaluate the right-hand side in the harmonic approximation, Eq. (4.26). This gives

$$
\begin{aligned}
C_{L}\left(\vec{q}_{\perp}, q_{\|}, \omega ;\left\{B_{i}^{0}\right\}\right) \\
\quad=\frac{\Delta_{*}\left[\omega-v_{0}\left(\ell_{0}^{*}\right) q_{\|} q_{\perp}^{z-\zeta}\right]^{2} q_{\perp}^{-(2 \chi+3 z+\zeta+d-1)}}{\mathcal{D}},
\end{aligned}
$$

where

$$
\begin{aligned}
& \mathcal{D}=\left\{\left[\frac{\omega}{q_{\perp}^{z}}-\omega_{+}\left(\ell_{*}\right)\right]^{2}\left[\frac{\omega}{q_{\perp}^{z}}-\omega_{-}\left(\ell_{*}\right)\right]^{2}\right. \\
&+\left[\left(\frac{\omega}{q_{\perp}^{z}}\right)\left[\Gamma_{L}\left(\ell_{*}\right)+\Gamma_{\rho}\left(\ell_{*}\right)\right]-v_{0}\left(\ell_{*}\right)\right. \\
&\left.\left.\times\left(\Gamma_{L}\left(\ell_{*}\right)+\frac{\gamma\left(\ell_{*}\right)}{v_{0}\left(\ell_{*}\right)} \Gamma_{\rho}\left(\ell_{*}\right)\right)\left(\frac{q_{\|}}{q_{\perp}^{\zeta}}\right)\right]^{2}\right\} \\
& \omega_{ \pm}\left(\ell_{*}\right) \equiv \omega_{ \pm}\left(\hat{q}_{\perp}, \frac{q_{\|}}{q_{\perp}^{\zeta}} ; \gamma\left(\ell_{*}\right), \rho_{0}\left(\ell_{*}\right),\left\{B_{i}\left(\ell_{*}\right)\right\}\right),
\end{aligned}
$$

with the sound frequencies $\omega_{ \pm}\left(\vec{q} ; \gamma, \rho_{0},\left\{B_{i}\right\}\right)$ obtained in the harmonic theory:

$$
\begin{aligned}
\omega_{ \pm}\left(\vec{q} ; \gamma, \rho_{0},\left\{B_{i}\right\}\right) \\
=\frac{\gamma+v_{0}}{2} q \cos \theta_{\vec{q}} \\
\quad \pm \sqrt{\left(\frac{\left(\gamma-v_{0}\right) q \cos \theta_{\vec{q}}}{2}\right)^{2}+\sigma_{1} \rho_{0} q^{2} \sin ^{2} \theta_{\vec{q}}} \\
=\frac{\left(\gamma+v_{0}\right) q_{\|}}{2} \pm \sqrt{\left(\frac{\left(\gamma-v_{0}\right) q_{\|}}{2}\right)^{2}+\sigma_{1} \rho_{0} q_{\perp}^{2}}
\end{aligned}
$$

$$
\Gamma_{L}\left(\ell_{*}\right) \equiv D_{L}^{*}+D_{\|}^{*}\left(\frac{q_{\|}}{q_{\perp}^{\zeta}}\right)^{2},
$$

$$
\Gamma_{\rho}\left(\ell_{*}\right) \equiv D_{\rho}^{*}\left(\frac{q_{\|}}{q_{\perp}^{\zeta}}\right)^{2},
$$

and $D_{L}^{*}, D_{\|}^{*}$, and $\Delta_{*}$ are the fixed point values of $D_{L}, D_{\|}$, and $\Delta$, to which those parameters will have flown for $\ell_{*}$ large, as it will be for small $q_{\perp}$.

The complication of this expression - that is, the fact that stops it from having a simple scaling form-is that the parameters $\gamma\left(\ell_{*}\right), \sigma_{1}\left(\ell_{*}\right)$, and $\rho_{0}\left(\ell_{*}\right)$ that appear implicitly in (5.44) do not flow to field point values for our "canonical' choice of $\chi, z$, and $\zeta$, as discussed earlier. Physically, this reflects the fact that the scaling of the sound speeds $(\omega$ $\propto q$ ) is different from that of their dampings (damping rate $\propto q^{2}$ in harmonic theory; we will show damping rate is $\propto q_{\perp}^{z}$ here, in a moment).

To proceed, it is first useful to reorganize Eq. (5.44) slightly; by multiplying numerator and denominator by $q_{\perp}^{4 z}$,

$$
\begin{aligned}
& C_{L}\left(\vec{q}_{\perp}, q_{\|}, \omega ;\left\{B_{i}^{0}\right\}\right) \\
& \quad=\frac{\Delta_{*}\left[\omega-v_{0}\left(\ell_{*}\right) q_{\|} q_{\perp}^{z-\zeta}\right]^{2} q_{\perp}^{(z-\zeta-2 \chi+1-d)}}{\left[\omega-q_{\perp}^{z} \omega_{+}\left(\ell_{*}\right)\right]^{2}\left[\omega-q_{\perp}^{z} \omega_{-}\left(\ell_{*}\right)\right]^{2}+\left[\omega q_{\perp}^{z}\left[\Gamma_{L}\left(\ell_{*}\right)+\Gamma_{\rho}\left(\ell_{*}\right)\right]-\left[v_{0}\left(\ell_{*}\right) \Gamma_{L}\left(\ell_{*}\right)+\gamma\left(\ell_{*}\right) \Gamma_{\rho}\left(\ell_{*}\right)\right] q_{\|} q_{\perp}^{2 z-\zeta}\right]^{2}} .
\end{aligned}
$$

Next, we solve the recursion relations for $\gamma\left(\ell_{*}\right), \sigma_{1}\left(\ell_{*}\right)$, and $\rho_{0}\left(\ell_{*}\right)$ :

$$
\begin{gathered}
v_{0}\left(\ell_{*}\right)=e^{(z-\zeta) \ell} * v_{0}(\ell=0)=v_{0}(0) q_{\perp}^{\zeta-z}, \\
\gamma\left(\ell_{*}\right)=e^{(z-\zeta) \ell} * \gamma(\ell=0)=\gamma(0) q_{\perp}^{\zeta-z}, \\
\sigma_{1}\left(\ell_{*}\right)=e^{(z-1) \ell} * \sigma_{1}(\ell=0)=\sigma_{1}(0) q_{\perp}^{1-z}, \\
\rho_{0}\left(\ell_{*}\right)=e^{(z-1) \ell} * \rho_{0}(\ell=0)=\rho_{0}(0) q_{\perp}^{1-z},
\end{gathered}
$$


where in the second equality in each equation we have used $\ell_{*}=\ln \left(1 /\left|\vec{q}_{\perp}\right|\right)$. Using these results and the expressions (5.46) and (5.47) for $\omega_{ \pm}\left(\ell_{*}\right)$, we see that $z$ and $\zeta$ drop out of the combinations

$$
\begin{aligned}
q_{\perp}^{z} \omega_{ \pm}\left(\ell_{*}\right) & =q_{\perp}^{z}\left[\frac{1}{2}\left[\gamma(0)+v_{0}(0)\right] \frac{q_{\|}}{q_{\perp}^{\zeta}} q_{\perp}^{\zeta-z} \pm \sqrt{\left.\left(\frac{\gamma(0)-v_{0}(0)}{2} \frac{q_{\|}}{q_{\perp}^{\zeta}} q_{\perp}^{\zeta-z}\right)^{2}+\sigma_{1}(0) \rho_{0}(0) q_{\perp}^{2(1-z)}\right]}\right. \\
& =\frac{1}{2}\left(\gamma+v_{0}\right) q_{\|} \pm \sqrt{\left(\frac{\left[\gamma(0)-v_{0}(0)\right] q_{\|}}{2}\right)^{2}+\sigma_{1}(0) \rho_{0}(0) q_{\perp}^{2}} \\
& =\omega_{ \pm}\left[\vec{q}_{\perp}, q_{\|} ; \gamma(0), v_{0}(0), \sigma_{1}(0), \rho_{0}(0)\right]
\end{aligned}
$$

and therefore the positions of the peaks in the full correlation function (5.50) are exactly those given by the harmonic theory using the bare parameters $\sigma_{1}(0), v_{0}(0), \gamma(0)$, and $\rho_{0}(0)$, namely, $w_{ \pm}\left[\vec{q} ; \gamma(0), v_{0}(0), \sigma_{1}(0), \rho_{0}(0)\right]$. This is a direct consequence of the fact that there are no (relevant) graphical renormalizations of the parameters $\left(\gamma, \sigma_{1}\right.$, and $\left.\rho_{0}\right)$ that determine the sound speeds [see the recursion relations (5.14)-(5.17)] and shows that the relevant nonlinearities below $d=4$ do not alter the positions of the peaks in the spatiotemporally Fourier-transformed velocity-velocity autocorrelations.

The same cannot, however, be said for their widths. Indeed, using the above result for the sound speeds and Eqs. (5.48) and (5.49), we see that $C_{L}(\vec{q}, \omega)$ can be rewritten:

$$
C_{L}(\vec{q}, \omega)=\frac{\Delta_{*}\left[\omega-v_{0}(0) q_{\|}\right]^{2} q_{\perp}^{(z-\zeta-2 \chi+1-d)}}{\left[\omega-c_{+}\left(\theta_{\vec{q}}\right) q\right]^{2}\left[\omega-c_{-}\left(\theta_{\vec{q}}\right) q\right]^{2}+\left\{\omega\left[\Gamma_{L}^{R}(\vec{q})+\Gamma_{\rho}^{R}(\vec{q})\right]-q_{\|}\left[v_{0}(0) \Gamma_{L}^{R}(\vec{q})+\gamma(0) \Gamma_{\rho}^{R}(\vec{q})\right]\right\}^{2}},
$$

where the sound speeds are given by the harmonic result equation (4.11), and the renormalized dampings

$$
\begin{gathered}
\Gamma_{L}^{R}(\vec{q})=\left[D_{\|}^{*}\left(\frac{q_{\|}}{q_{\perp}^{\zeta}}\right)^{2}+D_{L}^{*}\right] q_{\perp}^{z} \equiv q_{\perp}^{z} f_{L}\left(\frac{q_{\|}}{q_{\perp}^{\zeta}}\right), \\
\Gamma_{\rho}^{R}(\vec{q})=D_{\rho}^{*}\left(\frac{q_{\|}}{q_{\perp}^{\zeta}}\right)^{2} q_{\perp}^{z} \equiv q_{\perp}^{z} f_{\rho}\left(\frac{q_{\|}}{q_{\perp}^{\zeta}}\right)
\end{gathered}
$$

obey simple scaling laws.

The exact form of the scaling laws that we have obtained here (namely, e.g., $\left.f_{L}\left(q_{\|} / q_{\perp}^{\zeta}\right)=\left[D_{\|}^{*}\left(q_{\|} / q_{\perp}^{\zeta}\right)^{2}+D_{L}^{*}\right]\right)$, is not correct, because our choice of $\ell_{*}=\ln \left(1 / q_{\perp}\right)$ is only valid when $q_{\perp}^{\zeta} \gg q_{\|}$. In the opposite limit $q_{\perp}^{\zeta} \ll q_{\|}$, the fluctuations become negligible in the renormalized problem once $D_{\|} q_{\|}^{2}$ becomes $\gg D_{B} \Lambda^{2}$ in the renormalized problem, because at this point the linearized approximation to the correlation functions is smaller than its largest value at the Brillouin zone boundary. This means we can now stop the renormalization at $\ell_{*}$ such that $e^{\zeta} * q_{\|}=\Lambda \times D_{B}^{*} / D_{\|}^{*}$, which implies that $\ell_{*}=\ln \left(1 / q_{\|}\right) / \zeta+O(1)$, where the $O(1)$ factor is universal, because it depends only on the fixed point values of the diffusion constants. Performing the above calculations with this choice of $\ell_{*}$, we now obtain

$$
\Gamma_{L}^{R}(\vec{q})=\left[D_{\|}^{*}+D_{L}^{*}\left(\frac{q_{\perp}^{2}}{q_{\|}^{2 / \zeta}}\right)\right] q_{\|}^{z / \zeta} \times O(1) \equiv q_{\perp}^{z} f_{L}\left(\frac{q_{\|}}{q_{\perp}^{\zeta}}\right),
$$

where we have now defined $f_{L}\left(q_{\|} / q_{\perp}^{\zeta}\right) \equiv\left[D_{\|}^{*}\left(q_{\|} / q_{\perp}^{\zeta}\right)^{z / \zeta}\right.$ $\left.+D_{L}^{*}\left(q_{\perp}^{\zeta} / q_{\|}\right)^{2-z / \zeta}\right] \times O(1)$. Note that the precise form of this scaling function is different in this regime from that found earlier for $q_{\perp}^{\zeta} \gg q_{\|}$. Furthermore, its exact form is uncertain, due to our uncertainty in the $O(1)$ factor, which, as discussed earlier, cannot be determined without knowing the fixed-point values $D_{i}^{*}$ of the diffusion constants. However, regardless of their values, we still get a scaling law with the same power of $q_{\perp}$ and the same scaling variable $q_{\|} / q_{\perp}^{\zeta}$ as that found earlier in the opposite limit.

In between these two limits we have to choose $\ell_{*}$ to smoothly interpolate between the two limits. This choice will clearly depend on the ratio $q_{\|} / q_{\perp}^{\zeta}$. Naively, one could imagine simply choosing $\ell_{*}=\ln \left[\min \left(1 / q_{\perp}, O(1) / q_{\|}^{1 / \zeta}\right)\right]$. A subtler choice would take into account the $O(1)$ perturbative corrections we have neglected, and would presumably lead to a smooth crossover of $\ell_{*}$ between the two limits.

The moral of this discussion is threefold:

(1) We always get scaling laws of the form (5.57) for the dampings.

(2) The renormalized damping functions and the noise strength are always of such a form that they depend only on $q_{\|}$for $q_{\|} \gg q_{\perp}^{\zeta}$, and only on $q_{\perp}$ in the opposite limit.

(3) We can only calculate the scaling function if we know the diffusion constants at the fixed point.

This last point will stop us from calculating the crossover functions in $d=2$, even though, as we will see, we can calculate the exponents there.

We see from Eq. (5.57) that the physical significance of the exponent $z$ is that it gives the scaling of the peak widths (in $\omega$ ) of $C_{L}(\vec{q}, \omega)$ with $q_{\perp}$, while the peak positions continue to obey the " $z=1$ " scaling $\omega \propto q$.

Similar, but actually far simpler, arguments show that the transverse correlation function $C_{T}(\vec{q}, \omega)$ obeys

$$
C_{T}(\vec{q}, \omega)=\frac{f_{\Delta}\left(q_{\|} / q_{\perp}^{\zeta}\right) q_{\perp}^{z-\zeta-2 \chi+1-d}}{\left(\omega-\gamma q_{\|}\right)^{2}+\Gamma_{T}^{2}(\vec{q})}
$$


where $\Gamma_{T}(\vec{q})$ obeys the scaling law

$$
\Gamma_{T}(\vec{q})=q_{\perp}^{z} f_{T}\left(\frac{q_{\|}}{q_{\perp}^{\zeta}}\right),
$$

and $f_{\Delta}$ is a scaling function associated with $\Delta$. For general values of $q_{\perp}$ and $q_{\|}$, the same scaling function $f_{\Delta}$ should also be present in all of the other correlation functions as well (wherever $\Delta$ appears), such as Eq. (5.56) for the longitudinal correlation function.

Likewise, the propagators of the full nonlinear theory are given, in $d<4$, by the harmonic expressions (4.18)-(4.21), except that $\Gamma_{L}, \Gamma_{\rho}$, and $\Gamma_{T}$ in those expressions are replaced by the anharmonic scaling laws (5.57), (5.58), and (5.61).

To complete the specification of the scaling laws, we need the asymptotic behavior of the scaling functions $f_{\Delta, L, T, \rho}(u)$. From Eqs. (5.57), (5.58), and the analogous result for Eq. (5.61), and requiring that the second point of our tripartite moral applies, we see that

$$
f_{\Delta}(u) \propto \begin{cases}\text { const, } & u \rightarrow 0 \\ u^{(z-\zeta-2 \chi+1-d) / \zeta}, & u \rightarrow \infty\end{cases}
$$

and

$$
f_{L, T, \rho}(u) \propto \begin{cases}\text { const, } & u \rightarrow 0 \\ u^{z / \zeta}, & u \rightarrow \infty,\end{cases}
$$

which implies that

$$
\Gamma_{L, T, \rho}(\vec{q}) \propto \begin{cases}q_{\perp}^{z}, & q_{\|} \ll q_{\perp}^{\zeta} \\ q_{\|}^{z / \zeta}, & q_{\|} \gg q_{\perp}^{\zeta} .\end{cases}
$$

The simplest summary of the scaling of all correlation functions and propagators is as follows: simply use the harmonic expressions for them, except that diffusion constants $D_{T, B, \rho}$ should be replaced by wave-vector-dependent quantities that diverge as $\vec{q} \rightarrow 0$, according to the scaling law

$$
D_{T, B, \rho}(\vec{q})=q_{\perp}^{z-2} f_{T, B, \rho}\left(\frac{\left(q_{\|} / \Lambda\right)}{\left(q_{\perp} / \Lambda\right)^{\zeta}}\right),
$$

the bare noise strength $\Delta$ should be replaced by

$$
\Delta(\vec{q})=\Delta_{*}\left(\frac{q_{\perp}}{\Lambda}\right)^{z-\zeta-2 \chi+1-d} f_{\Delta}\left(\frac{\left(q_{\|} / \Lambda\right)}{\left(q_{\perp} / \Lambda\right)^{\zeta}}\right)
$$

and the diffusion constant $D_{\|}$should be replaced by

$$
D_{\|}(\vec{q})=q_{\perp}^{z-2 \zeta} f_{\|}\left(\frac{\left(q_{\|} / \Lambda\right)}{\left(q_{\perp} / \Lambda\right)^{\zeta}}\right)
$$

as can be seen by requiring that

$$
D_{\|}(\vec{q}) q_{\|}^{2}=D_{\|}^{*}\left(\frac{q_{\|}}{q_{\perp}^{\zeta}}\right)^{2} q_{\perp}^{z}
$$

the right-hand side being the form of the $q_{\|}$-dependent term in Eq. (5.57).
We hope the reader has not been too confused by the fact that we have restored the ultraviolet cutoff $\Lambda \sim 1 / \ell_{0}$ to the problem by going back to dimensionful units where $\Lambda \neq 1$.

This completes our discussion of how the renormalization group, and, in particular, the exponents $z, \chi$, and $\zeta$, relate to physically observable correlation functions and propagators. Now, we turn to the problem of actually calculating those exponents.

\section{Exponents in $d=2$}

To do this, we must calculate the graphical corrections in Eqs. (5.11)-(5.16). The procedure for this, as discussed in [6] involves the harmonic correlation functions and propagators and vertices representing the nonlinearities $\lambda_{1,2, \rho}$ and $\sigma_{2}$. Rather than actually calculating these corrections, we will show that, when $\lambda_{2}=0$, the structure of the theory is such that we can determine the exponents $\chi, z$, and $\zeta$ exactly.

Consider first the $\lambda_{1}$ nonlinearity. Separating $\vec{v}_{\perp}$ into transverse and longitudinal components,

$$
\vec{v}_{\perp} \equiv \vec{v}_{T}+\vec{v}_{L}
$$

this can be written as

$$
\begin{aligned}
\left(\vec{v}_{\perp} \cdot \vec{\nabla}_{\perp}\right) \vec{v}_{\perp}= & \left(\vec{v}_{T} \cdot \vec{\nabla}_{\perp}\right) \vec{v}_{T}+\left(\vec{v}_{L} \cdot \vec{\nabla}_{\perp}\right) \vec{v}_{L}+\left(\vec{v}_{T} \cdot \vec{\nabla}_{\perp}\right) \vec{v}_{L} \\
& +\left(\vec{v}_{L} \cdot \vec{\nabla}_{\perp}\right) \vec{v}_{T}
\end{aligned}
$$

Now consider the graphs that can be constructed from $\vec{v}_{L}$ $-\vec{v}_{T}$, the cross terms in this expression. These will always mix transverse and longitude propagators and correlation functions in the internal integrals over momentum and frequency. But, as noted earlier in our discussion of the harmonic theory, the peaks in the longitudinal propagators and correlation functions occur at different frequencies $[\omega$ $\left.=\omega_{ \pm}(\vec{q})\right]$ than those in the transverse propagators and correlation function, which occur at $\omega=0$. Furthermore, the overlap between these peaks is negligible, since their widths $\left(\propto q_{\perp}^{z}\right.$ with $\left.z>1\right)$ are much less than this offset in peak positions. This implies that the integral over wave vectors and frequencies of any graph that mixes transverse and longitudinal propagators and correlation functions will be much less (by powers of $q$ ) than any similar graph containing purely transverse or purely longitudinal propagators and correlation functions. Hence, the $\vec{v}_{L}-\vec{v}_{T}$ cross terms in Eq. (5.70) are irrelevant compared to the pure $\vec{v}_{L}$ and $\vec{v}_{T}$ terms.

Now let us consider those relevant pieces. The Fourier transform of the $\vec{v}_{T}$ piece at wave vector $\vec{q}$ can be written in Fourier space:

$$
\begin{aligned}
\operatorname{FT}\left[\left(\vec{v}_{T} \cdot \vec{\nabla}_{\perp}\right) v_{T_{i}}\right]_{q} & =i \sum_{\vec{p}} \vec{v}_{T}(\vec{p})\left(\vec{q}_{\perp}-\vec{p}_{\perp}\right) v_{T_{i}}(\vec{q}-\vec{p}) \\
& =i q_{j}^{\perp} \sum_{\vec{p}} v_{T_{j}}(\vec{p}) v_{T_{i}}(\vec{q}-\vec{p})
\end{aligned}
$$

where we have used the fact that $\vec{v}_{T}$ is transverse, so $\vec{p}_{\perp} \cdot \vec{v}_{T}(\vec{p})=0$. So this piece of the $\lambda_{1}$ vertex, which is a term 


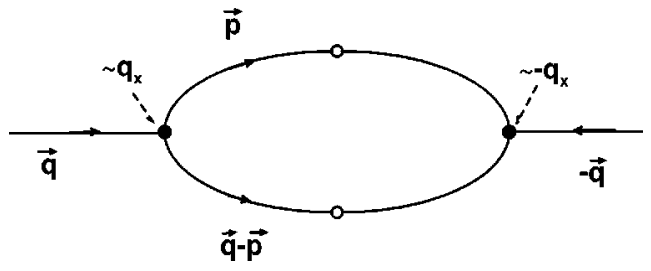

FIG. 7. Feynmann graph renormalizing the noise correlations when $\lambda_{2}=0$. There is a factor of the external momentum $\left|\vec{q}_{\perp}\right|$ $\equiv q_{x}$ associated with each vertex; hence this graph does not renormalize $\Delta$, but, rather, only changes $O\left(q^{2}\right)$ pieces of the $f-f$ correlation function.

in the equation for $\partial_{t} v_{i}(\vec{q}, t)$, is proportional to the external momentum $\vec{q}_{\perp}$. So is the purely longitudinal term, as can easily be seen in real space. Since $\vec{v}_{L}$ is longitudinal, we can write

$$
\vec{v}_{L}=\vec{\nabla}_{\perp} \phi
$$

for some scalar field $\phi$. Now the second term in Eq. (5.70) can be rewritten in terms of $\phi$ :

$\left(\vec{v}_{L} \cdot \vec{\nabla}_{\perp}\right) v_{L i}=\left(\partial_{j} \phi\right)\left(\partial_{j} \partial_{i} \phi\right)=\left(\partial_{j} \phi\right)\left(\partial_{i} \partial_{j} \phi\right)=\frac{1}{2} \partial_{i}\left(\partial_{j} \phi \partial_{j} \phi\right)$,

which is clearly a total derivative, whose Fourier transform is proportional to $\vec{q}_{\perp}$.

Hence, the two relevant pieces of the $\lambda_{1}$ vertex are proportional to the external momentum $\vec{q}_{\perp}$. Clearly, the $\sigma_{2}$ term, being a total $\perp$ derivative, is also proportional to $\vec{q}_{\perp}$ in Fourier space. Hence, when $\lambda_{2}=0$, all the remaining relevant vertices are proportional to $\vec{q}_{\perp}$. An immediate consequence of this is that $\Delta$ and $D_{\|}$acquire no graphical renormalization. For $\Delta$, this can be seen by noting that any graph that renormalizes $\Delta$ (e.g., Fig. 7) must contain two external vertices, each proportional to $q_{\perp}$, and hence must be proportional to $q_{\perp}^{2}$. Therefore all renormalizations of $\Delta$ must be proportional to $q_{\perp}^{2}$, and hence negligible, as $|\vec{q}| \rightarrow 0$, relative to the bare $\Delta$. Likewise, any graph for the diffusion constants must be proportional to Fig. 8, which must be proportional to at least one power of $\vec{q}_{\perp}$. Since $D_{\|}$and $D_{\rho}$ involve no powers of $\vec{q}_{\perp}$, they cannot be renormalized graphically.

Thus, when $\lambda_{2}=0, \Delta, D_{\rho}$, and $D_{\|}$get no graphical renormalization. That is, $G_{\|}, G_{\rho}$, and $G_{\Delta}$ in Eqs. (5.12) and (5.16) are, exactly, $=0$. Thus, the requirement that $\Delta, D_{\|}$, and $D_{\rho}$ flow to fixed points $\left(d D_{\|, \rho} / d \ell\right)=0=d \Delta / d \ell$ leads

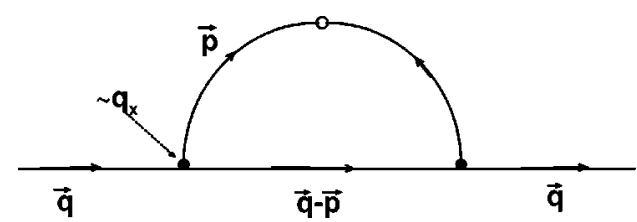

FIG. 8. Feynmann graph for diffusion constants. When $\lambda_{2}=0$, this graph is proportional to at least one power of $\left|\vec{q}_{\perp}\right| \equiv q_{x}$, and, so, cannot renormalize $D_{\|}$or $D_{\rho}$. to two independent exact scaling relations between the three independent exponents $\chi, z$, and $\zeta$. Requiring $d D_{\|, \rho} / d \ell$ $=0$ implies

$$
z=2 \zeta,
$$

while requiring $d \Delta / d \ell=0$ leads to

$$
z=\zeta+2 \chi+d-1
$$

We emphasize that we have only shown that these relations (5.74) and (5.75) hold when $\lambda_{2}=0$.

We can obtain a third independent exact scaling relation between these three exponents, and thereby determine them exactly, when $\lambda_{2}=0$, by considering the renormalization of the nonlinearities $\lambda_{1}, \lambda_{\rho}$, and $\sigma_{2}$.

We start deriving this third relation by noting that when $\lambda_{2}=0$ and $\lambda_{1}=\lambda_{\rho} \equiv \lambda$, there can be $n o$ graphical renormalization of $\lambda_{1}$. This is because for these parameter values the equations of motion (3.3) and (3.4) have an exact symmetry that we call pseudo-Galilean invariance: namely, they remain unchanged under the "boost" transformation:

$$
\begin{gathered}
\vec{r}_{\perp} \rightarrow \vec{r}_{\perp}-\lambda \vec{v}_{b} t, \\
\vec{v}_{\perp}(\vec{r}, t) \rightarrow \vec{v}_{\perp}(\vec{r}, t)+\vec{v}_{b},
\end{gathered}
$$

where the "boost" velocity $\vec{v}_{b}$ is an arbitrary constant vector in the $\perp$ plane.

This symmetry must be preserved upon renormalization with the same value of $\lambda$. Hence, there can be no graphical renormalization of $\lambda$, when $\lambda_{1}=\lambda_{\rho}$. That is, $G_{1}^{\lambda}=0$ when $\lambda_{1}=\lambda_{\rho}$. Since $G_{\rho}^{\lambda}=0$ always, it is clear that, if $\lambda_{1}<\lambda_{\rho}$ initially, it will always remain so upon renormalization.

This implies that, for flocks that start with bare $\lambda_{1}$ less than the bare $\lambda_{\rho}$ (which should be a finite fraction of all possible flocks), only two types of fixed points are possible: (I) $\lambda_{1}^{*}=\lambda_{\rho}^{*}=0$ or (II) $\lambda_{\rho}^{*} \neq 0$.

The first type of fixed point (I), however, is readily seen to be unstable to $\lambda_{\rho}$, which must always be nonzero (and, in fact, =1) initially. Hence, this fixed point is never reached, and we must flow to a fixed point of type II. To see that fixed points of type I are unstable, note that for such a fixed point, the only remaining relevant nonlinearity is $\sigma_{2}$. But, by itself, $\sigma_{2}$ cannot renormalize any of the diffusion constants $D_{B}$ and $D_{i}$. The reason for this is that any graph (e.g., Fig. 8) that renormalizes any diffusion constant must have an external velocity leg emerging from the right. However, using only the $\sigma_{2}$ vertex, which only involves $\rho$, we can only make graphs with a $\rho$ leg emerging from the right. Therefore, at a fixed point of type I, $G_{B, T}=0$, exactly, in Eq. (5.11). Thus, to find a fixed point for $D_{B, T}$, we must choose $z=2$. Combining this with the previous exact scaling relations (5.74) and (5.75), we find $\zeta=1$ and $\chi=1-d / 2$. But using these values (which are nothing but those that we found in the harmonic theory), we find that $\lambda_{\rho}$ is a relevant perturbation at any fixed point of type I:

$$
\frac{d \lambda_{\rho}}{d l}=\left(2-\frac{d}{2}\right) \lambda_{\rho}
$$


for all spatial dimensions $d<4$. Note that (5.78) is exact at any fixed point of type I, since $G_{\rho}^{\lambda}=0$, exactly.

So fixed points of type I are unstable, and we will always flow to a fixed point of type II. Using the recursion relation (5.15) for $\lambda_{\rho}$, and the fact that $G_{\rho}^{\lambda}=0$ always, we immediately obtain that $\lambda_{\rho}^{*}$ can be $\neq 0$ if and only if a third exact scaling relation is satisfied, namely,

$$
\chi=1-z .
$$

The three relations (5.74), (5.75), and (5.79) that hold when $\lambda_{2}=0$ can be solved trivially, to find the exact scaling exponents in all $d<4$ that describe flocks with $\lambda_{2}=0$ :

$$
\begin{gathered}
\zeta=\frac{d+1}{5}, \\
z=\frac{2(d+1)}{5},
\end{gathered}
$$

and

$$
\chi=\frac{3-2 d}{5}
$$

Note that these match continuously, at the upper critical dimension $d=4$, onto their harmonic values $\zeta=1, z=2$, and $\chi=1-d / 2=-1$, as they should.

If $\lambda_{1}^{\text {Bare }}>\lambda_{\rho}^{\text {Bare }}$, then, besides the two cases that we discussed above, there may be a third type of fixed point: (III) $\lambda_{\rho}^{*}=0, \lambda_{1}^{*} \neq 0$.

For this type of fixed point to be stable, the exponents $\chi$ and $z$ have to satisfy

$$
\chi+z<1 .
$$

The above inequality, together with Eq. (5.74) and Eq. (5.75), give Eqs. (5.80), (5.81), and (5.82) with the = signs replaced by $<$. For simplicity, we will only discuss the cases with $\lambda_{1}^{\text {Bare }}<\lambda_{\rho}^{\text {Bare }}$, where the exponents are given by Eqs. (5.80), (5.81), and (5.82). However, all the qualitative results will be valid for case III, e.g., the spontaneous symmetry broken phase will be more stable in case III if it is stable in case II because of the inequality. The simplest possible scenario is that there is only one stable fixed point, regardless of whether $\lambda_{\rho}^{\text {Bare }}<\lambda_{1}^{\text {Bare }}$ or not, and that it is type II, and has the canonical exponents (5.80)-(5.82). We consider it highly probable that this is, in fact, the case. Even if it is not, Eqs. (5.80)-(5.82) do hold for some flocks (those with $\lambda_{\rho}^{\text {Bare }}$ $\left.>\lambda_{1}^{\text {Bare }}\right)$.

Our derivation of these results (5.80)-(5.82) depended only on the assumption that $\lambda_{2}^{*}=0$. Note, however, that in $d=2$, any flock is equivalent to a flock with $\lambda_{2}=0$. This is because the $\lambda_{1}$ and $\lambda_{2}$ vertices become identical in $d=2$, where $\vec{v}_{\perp}$ has only one component, which we will take to be $x$. That is, in $d=2$,

$$
\begin{gathered}
\lambda_{1}\left(\vec{v}_{\perp} \cdot \vec{\nabla}_{\perp}\right) \vec{v}_{\perp}=\lambda_{1} \hat{x} v_{x} \partial_{x} v_{x}=\frac{1}{2} \lambda_{1} \partial_{x}\left(v_{x}^{2}\right) \hat{x}, \\
\lambda_{2}\left(\vec{\nabla}_{\perp} \cdot \vec{v}_{\perp}\right) \vec{v}_{\perp}=\lambda_{2} \hat{x}\left(\partial_{x} v_{x}\right) v_{x}=\frac{1}{2} \lambda_{2} \partial_{x}\left(v_{x}^{2}\right) \hat{x}
\end{gathered}
$$

so that the full $\vec{v}_{\perp}$ nonlinearity becomes $\frac{1}{2}\left(\lambda_{1}+\lambda_{2}\right)$ $\times \partial_{x}\left(v_{x}^{2}\right) \hat{x}$, which is just what we would get if we started with a (primed) model with $\lambda_{2}^{\prime}=0$ and $\lambda_{1}^{\prime}=\lambda_{1}+\lambda_{2}$. This later model, since it has $\lambda_{2}^{\prime}=0$, must have the "canonical" exponents (5.80)-(5.81) and, hence, so must the $\left(\lambda_{1}, \lambda_{2}\right)$ model, which includes all possible $d=2$ models. So all models in $d=2$ must have the canonical exponents (5.80)(5.82).

Equivalently, we can derive this result by simply noting that, in $d=2$, the full $v_{\perp}-v_{\perp}$ vertex becomes $\frac{1}{2}\left(\lambda_{1}\right.$ $\left.+\lambda_{2}\right) \partial_{x}\left(v_{x}^{2}\right)$, which is a total $x$ derivative even when $\lambda_{2}$ $\neq 0$. Furthermore, the $d=2$ model now has the pseudoGalilean invariance (5.76) and (5.77) when $\lambda_{1}+\lambda_{2}=\lambda_{\rho}$. These two properties $\left(v_{\perp}-v_{\perp}\right.$ vertex total $x$ derivative, and pseudo-Galilean invariance at a special point) are all that we used to derive the "canonical" exponent (5.80)-(5.82); so those canonical exponents must hold in $d=2$. Setting $d=2$ in Eqs. (5.80)-(5.82), we obtain

$$
\begin{gathered}
\zeta=\frac{3}{5}, \\
z=\frac{6}{5}, \\
\chi=-\frac{1}{5} .
\end{gathered}
$$

Note, in particular, that $\chi<0$. This implies, as discussed earlier, that the flock exhibits true long-ranged order.

Using the exponents (5.86)-(5.88) in the general scaling relations, such as Eqs. (5.31) and (5.33), we obtain all of the scaling results for correlation functions in $d=2$ quoted in the Introduction. Note also that for this set of exponents $z-\zeta$ $-2 \chi+1-d=0$. Hence, from Eq. (5.66), we see that the noise strength $\Delta$ is a constant, independent of $\vec{q}$, which makes sense since $\Delta$ is unrenormalized graphically. So, in the $d=2$ model, we can calculate all correlation functions from their harmonic expressions, except that we replace the diffusion constants $D_{B, T, \rho}$ with functions that diverge as $\vec{q}$ $\rightarrow 0$ according to the scaling laws

$$
D_{B, T}(\vec{q})=q_{\perp}^{-4 / 5} f_{B, T}\left(\frac{\left(q_{\|} / \Lambda\right)}{\left(q_{\perp} / \Lambda\right)^{3 / 5}}\right),
$$

where we have used the exact $d=2$ exponents $z=\frac{6}{5}$ and $\zeta$ $=\frac{3}{5}$ in the general scaling law (5.65). $D_{\|, \rho}$, on the other hand, are, like $\Delta$, constants, since $z=2 \zeta$ [see general equation (5.12)], which also makes sense since $D_{\|, \rho}$ are unrenormalized graphically. Hence, the only replacement needed to turn the harmonic results into the correct results for the full, nonlinear theory in $d=2$ is Eq. (5.89).

\section{D. $d>2$}

Now we turn to $d>2$. Here the canonical exponents need not hold if $\lambda_{2}^{*} \neq 0$. The obvious thing to do, therefore, is to determine whether a small $\lambda_{2}$ is a relevant or irrelevant perturbation at the $\lambda_{2}=0$ fixed point. We have attempted to do this to leading (one-loop) order in a $4-\epsilon$ expansion. This involves calculating the perturbative corrections $G_{1,2}^{\lambda}$ and $G_{\sigma_{2}}$ in the recursion relations (5.15)-(5.13) to one-loop order, and to linear order in $\lambda_{2}$. Once this is done, we can find 
a fixed point with $\lambda_{2}=0$, and then calculate the linear renormalization-group eigenvalue of $\lambda_{2}$ at this fixed point. That is, we will expand the right hand side of the recursion relation (5.15) for $\lambda_{2}$ to linear order in $\lambda_{2}$, obtaining

$$
\frac{d \lambda_{2}}{d l}=\gamma_{2} \lambda_{2}
$$

for $\lambda_{1}=\lambda_{1}^{*}, \lambda_{\rho}=\lambda_{\rho}^{*}, \sigma_{2}=\sigma_{2}^{*}$, and $\lambda_{2}$ small. If $\gamma_{2}$ is $<0$, then the $\lambda_{2}=0$ fixed point is (at least locally) stable, and the canonical exponents (5.80) $-(5.82)$ will hold for all $d$. Unfortunately, an (extremely laborious) calculation (involving 14 different Feynmann graphs) shows, after many seemingly miraculous and unexpected cancellations between different graphs, that $G_{1}^{\lambda}, G_{2}^{\lambda}$, and $G_{\sigma_{2}}$ are exactly zero to one loop order. This implies that

$$
\chi+1-z=0\left(\epsilon^{2}\right)
$$

and that, to this order at least, $\lambda_{1,2, \rho}$ and $\sigma_{2}$ can take on any value at the fixed point. That is, to this order, there appears to be a fixed "line" [actually, a fixed four-dimensional subspace $\left.\left(\lambda_{1}, \lambda_{2}, \lambda_{\rho}, \sigma_{2}\right)\right]$, instead of a single fixed point. This, unfortunately, eliminates all of our predictive power for the exponents. For example, keeping $D_{\|}$fixed leads to

$$
z-2 \zeta=-G_{\|}\left(\lambda_{1}, \lambda_{2}, \lambda_{\rho}, \sigma_{2}\right)
$$

Our earlier arguments show that $G_{\|}$vanishes if $\lambda_{2}$ does; however, to this order $\lambda_{2}^{*}$ can be anything; hence, so can $G_{\|}$, and so we get no information about $z$ and $\zeta$ from this relation at all. Likewise the recursion relation for $\Delta$ leads to

$$
z-\zeta-2 \chi+1-d=-G_{\Delta}\left(\lambda_{2}, \lambda_{1}, \lambda_{\rho}, \sigma_{2}\right)
$$

with the right-hand side again vanishing if $\lambda_{2}=0$, but taking on any value you like if $\lambda_{2}$ can be anything, as it can, to this order.

So what actually happens for $2<d<4$ ? Unfortunately, from our one-loop calculation, we cannot say, but can only enumerate the possibilities:

Possibility I: At higher order, $\lambda_{2}$ proves to be irrelevant, and flows to zero at the fixed point. In this case, the canonical exponents (5.80)-(5.82) will hold, for all flocks, in all $d$ in the range $2<d<4$.

Possibility II: $d \lambda_{2} / d \ell=0$ to all orders (i.e., exactly). In this case, there is a fixed line (or, more generally, $D$ dimensional subspace with $D \geqslant 1$ ) with exponents that vary as continuous functions of $\lambda_{2}$, which can take on any value. Hence, the exponents $\chi, z$, and $\zeta$ will be continuously variable functions of the parameters in the ordered phase. This behavior is somewhat reminiscent of that of the $d=2$ equilibrium $X-Y$ model, although here it is occurring for an entire range of spatial dimension $2<d<4$, and, furthermore, is not associated in any way with the absence of true longranged orientational order, since such order is actually present in our model.

Possibility III: $d \lambda_{2} / d \ell>0$ at higher order, and the ordered phase is controlled by a new, $\lambda_{2}^{*} \neq 0$ fixed point. In this case, the exponents will again be universal, but presumably, different from the canonical ones (5.80)-(5.82). Unfortunately, we have no idea what they will be.
We should emphasize that all flocks will be described by only one of the three possibilities enumerated above (i.e., one cannot have different possibilities realized in different flocks). Unfortunately, we have no idea which of the above possibilities is realized for $2<d<4$.

\section{ANISOTROPIC MODEL}

Not all flocks, of course, are equally likely to move in any direction in the space they occupy. Flocks of birds, for instance, although they occupy a $d=3$ dimensional volume (the air), are far more likely to move horizontally than vertically. This is presumably because gravity breaks the rotational symmetry between the horizontal plane and vertical directions.

One can imagine a variety of "microscopic" rules, like the Vicsek rule described earlier, that would exhibit such anisotropy. For example, one could apply a "Vicsek' rule in three dimensions, selecting thereby a vector $\hat{n}$. Instead of moving along that vector, however, one could instead move along a vector "compressed" along some $(z)$ axis

$$
\vec{n}^{\prime}=s n_{z} \hat{z}+\vec{n}_{\perp}
$$

with $s<1$ and $\vec{n}_{\perp}=\hat{n}-n_{z} \hat{z}$. This will tend to promote motion in the $x-y$ plane at the expense of motion in the $z$ direction. Alternatively, one could project all velocities into the $x-y$ plane, apply a Vicsek rule to them (while still sampling neighbors in three dimensions), and then add to this $x y$ move a random decorrelated step in the $z$ direction [10].

For technical reasons that will, we hope, become obvious, we will focus our attention on systems that, whatever their spatial dimension $d$, have an easy plane of motion; i.e., two components of velocity that are intrinsically favored over the other $d-2$. We will also assume perfect isotropy within this plane and within the $d-2$ dimensional "hard"' subspace. The case of birds flying horizontally corresponds to $d=3$.

A natural extension of our fully isotropic model (EOM) to this case is

$$
\begin{aligned}
\partial_{t} \vec{v}+ & \lambda_{1}(\vec{v} \cdot \vec{\nabla}) \vec{v}+\lambda_{2}(\vec{\nabla} \cdot \vec{v}) \vec{v}+\lambda_{3} \vec{\nabla}\left(|\vec{v}|^{2}\right) \\
= & -\vec{\nabla} P(\rho)+\alpha \vec{v}-\beta|\vec{v}|^{2} \vec{v}-\delta \alpha \vec{v}_{H}+D_{B} \vec{\nabla}(\vec{\nabla} \cdot \vec{v}) \\
& +D_{T}^{e} \nabla_{e}^{2} \vec{v}+D_{T}^{H} \nabla_{H}^{2} \vec{v}+D_{2}(\vec{v} \cdot \vec{\nabla})^{2} \vec{v}+\vec{f}
\end{aligned}
$$

Mass conservation, of course, still applies:

$$
\partial_{t} \rho+\vec{\nabla} \cdot(\rho \vec{v})=0
$$

and the pressure $P(\rho)$ will still be given by the same expansion in $\delta \rho=\rho-\rho_{o}$

$$
P(\rho)=\sum_{n=1}^{\infty} \sigma_{n}(\delta \rho)^{n} .
$$

In Eq. (6.2), $\vec{v}_{H}$ denotes the $d-2$ "hard" components of $\vec{v}$, i.e., those orthogonal to the $d=2$ easy plane. Likewise, $\nabla_{e}^{2}$ and $\nabla_{H}^{2}$ denote the operators $\sum_{i=1}^{2} \partial^{2} / \partial x_{i}^{2}$ and $\sum_{i=3}^{d} \partial^{2} / \partial x_{i}^{2}$, respectively, where $i=1,2$ are the "easy" Cartesian direc- 
tions, and $i=3 \rightarrow d$ the "hard" ones. The term $-\delta \alpha\left|\vec{v}_{H}\right|^{2}$ $\delta \alpha>0$ suppresses these components of velocity relative to those in the easy plane.

Equation (6.2) is not, of course, the most general anisotropic model we could write down. For instance, one could have anisotropy in the nonlinear terms: e.g., terms like $\left(\vec{v}_{e} \cdot \vec{\nabla}\right) \vec{v}_{e}$ could have different coefficients than $\left(\vec{v}_{H}\right.$ $\cdot \vec{\nabla}) \vec{v}_{H}$. However, because $\vec{v}_{H}$ winds up being "massive," in the sense of decaying to zero too rapidly (i.e., nonhydrodynamically) at long wavelengths and times to nonlinearly affect the hydrodynamic (long wavelength, long time) behavior of the flock (in its low-temperature phase), any additional terms in Eq. (6.2) distinguishing $\vec{v}_{H}$ and $\vec{v}_{e}$ will have no effect on the hydrodynamic behavior in the low"temperature", phase. That is, Eq. (6.2) already contains enough anisotropy to generate all possible relevant, symmetry allowed terms in the broken symmetry state. Hence, we will keep things simple and not generalize Eq. (6.2) further.

As we did for the isotropic problem, we will now break the symmetry of this model, i.e., look for solutions to the form

$$
\vec{v}(\vec{r}, t)=\langle\vec{v}\rangle+\delta \vec{v}(\vec{r}, t)
$$

Now, however, the direction of the mean velocity $\langle\hat{v}\rangle$ [which we will choose as before, to be a static, spatially uniform solution of the noiseless $(\vec{f}=0)$ version of $(6.2)]$ is not arbitrary, but must lie in the easy $(1,2)$ plane. To see this, let us, without loss of generality, write

$$
\langle\vec{v}\rangle=v_{0 y} \hat{y}+v_{0 z} \hat{z}
$$

with $v_{0 y}$ and $v_{0 z}$ constants, $\hat{y}$ in the easy plane and $\hat{z}$ one of the $d-2$ " "hard" directions. To solve Eq. (6.2) with $\vec{f}=0$, these must obey

$$
\alpha v_{0 y}-\beta\left(v_{0 y}^{2}+v_{0 z}^{2}\right) v_{0 y}=0
$$

and

$$
(\alpha-\delta \alpha) v_{0 z}-\beta\left(v_{0 y}^{2}+v_{0 z}^{2}\right) v_{0 z}=0
$$

Subtracting $v_{0 y} \times(6.8)$ from $v_{0 z} \times \quad$ (6.7) we obtain $\delta \alpha v_{0 y} v_{0 z}=0$, which implies that either $v_{0 y}$ or $v_{0 z}$ must be zero. It is straightforward to show that the former solution is unstable (with two linear eigenvalues $\alpha>0$ ) to small $\vec{v}_{e}$ fluctuations, while the latter is stable (with $d-2$ linear eigenvalues $-\delta \alpha<0)$ to $\vec{v}_{H}$ fluctuations, so the solution with $\langle\vec{v}\rangle$ in the easy plane is the stable one. Furthermore, fluctuations in the "hard" directions are "massive,' in the sense of decaying rapidly to zero even at long wavelengths, and so can be neglected in the low-temperature phase (just like $v_{\|}$fluctuations in the isotropic case). Likewise, if we take

$$
\langle\vec{v}\rangle=v_{0} \hat{y}
$$

fluctuations in $\delta v_{y}=v_{y}-v_{0}$, will also be massive (with linear eigenvalue $-2 \alpha$ ). Eliminating the massive fields $\delta v_{y}$ and $\vec{v}_{H}$ in favor of the pressure, as we did for $\delta v_{\|}$in the isotropic case, gives

$$
\begin{gathered}
\delta v_{y}=-D_{\rho y} \partial_{y} \rho, \\
\vec{v}_{H}=-D_{\rho H} \vec{\nabla}_{H} \rho,
\end{gathered}
$$

where we have defined the diffusion constants

$$
\begin{gathered}
D_{\rho y} \equiv \frac{\sigma_{1}}{2 \alpha}, \\
D_{\rho H} \equiv \frac{\sigma_{1}}{\delta \alpha}
\end{gathered}
$$

and we have used the relation (6.4) for the pressure, and dropped all but the leading order linear terms in $\delta \rho$, since higher powers of $\delta \rho$ in Eqs. (6.10) and (6.11) prove to be irrelevant.

Using the solutions (6.10) and (6.11), and taking, for the reasons just discussed,

$$
\vec{v}(\vec{r})=\left[v_{0}+\delta v_{y}(\vec{r}, t)\right] \hat{y}+v_{x}(\vec{r}, t) \hat{x}+\vec{v}_{H}(\vec{r}, t)
$$

we can write a closed system of equations for $v_{x}(\vec{r}, t)$ and $\delta \rho(\vec{r}, t)$ :

$$
\partial_{t} \delta \rho+v_{0} \partial_{y} \delta \rho+\partial_{x}\left(\rho v_{x}\right)=\left(D_{\rho y} \partial_{y}^{2}+D_{\rho H} \nabla_{H}^{2}\right) \delta \rho,
$$

$$
\begin{aligned}
\partial_{t} v_{x}+\gamma \partial_{y} v_{x}+\frac{\lambda}{2} \partial_{x}\left(v_{x}^{2}\right)= & -\sigma_{1} \partial_{x}(\delta \rho)-\sigma_{2} \partial_{x}(\delta \rho)^{2}+\left(D_{\|} \partial_{y}^{2}\right. \\
& \left.+D_{x} \partial_{x}^{2}+D_{H} \nabla_{H}^{2}\right) v_{x}+f_{x}
\end{aligned}
$$

where we have defined $\lambda \equiv \lambda_{1}+\lambda_{2}$, and $\gamma=\lambda_{1} v_{0}$, and dropped irrelevant terms.

Proceeding as we did in the isotropic model, we begin by linearizing these equations, Fourier transforming them, and determining their mode structure.

The result of the first two steps is the Fourier-transformed equations of motion

$$
\left[-i\left(\omega-v_{0} q_{y}\right)+\Gamma_{\rho}(\vec{q})\right] \delta \rho(\vec{q}, \omega)+i q_{x} \rho_{0} v_{x}(\vec{q}, \omega)=0,
$$

$\left[-i\left(\omega-\gamma q_{y}\right)+\Gamma_{v}(\vec{q})\right] v_{x}(\vec{q}, \omega)+i \sigma_{1} q_{x} \delta \rho(\vec{q}, \omega)=f_{x}(\vec{q}, \omega)$

where we have defined

$$
\begin{gathered}
\Gamma_{\rho}(\vec{q}) \equiv D_{\rho y} q_{y}^{2}+D_{\rho H} q_{H}^{2}, \\
\Gamma_{v}(\vec{q}) \equiv D_{\|} q_{y}^{2}+D_{H} q_{H}^{2}+D_{x} q_{x}^{2} .
\end{gathered}
$$

Again as in the isotropic model, we first determine the eigenfrequencies $\omega(\vec{q})$ of these equations, finding

$$
\omega_{ \pm}(\vec{q})=c_{ \pm}\left(\theta_{\vec{q}}, \phi_{\vec{q}}\right) q-i \epsilon_{ \pm}(\vec{q})
$$

where the sound speeds

$$
c_{ \pm}\left(\theta_{\vec{q}}, \phi_{\vec{q}}\right)=\frac{1}{2}\left(\gamma+v_{0}\right) \cos \theta_{\vec{q}} \pm c_{2}\left(\theta_{\vec{q}}, \phi_{\vec{q}}\right)
$$

with 


$$
c_{2}\left(\theta_{\vec{q}}, \phi_{q}\right) \equiv \sqrt{\frac{1}{4}\left(\gamma-v_{0}\right)^{2} \cos ^{2} \theta_{q}+\sigma_{1} \rho_{0} \sin ^{2} \theta_{\vec{q}} \cos ^{2} \phi_{q}},
$$

where $\theta_{\vec{q}}$ is the polar angle between $\vec{q}$ and the $y$ axis, and $\phi_{q}$ is the azimuthal angle, measured relative to the $x$ axis, i.e., the angle between the projection of $\vec{q}$ orthogonal to $y$, and the $x$ axis.

A polar plot of this sound speed versus $\theta_{q}$ for $\phi_{\vec{q}}=0$ [i.e., $\vec{q}$ in the "easy" (i.e., $x-y$ ) plane] looks exactly like that for the isotropic model (Fig. 2). Indeed, any slice with fixed $\phi_{q}$ looks qualitatively like that figure, although, as $\phi_{q} \rightarrow \pi / 2$ (i.e., as $\vec{q}_{\perp}$, the projection of $\vec{q}$ orthogonal to $y$, approaches orthogonality to the $x$ axis), the sound velocity profile becomes two circles with their centers on the $y$ axis and both circles passing through the origin. by

The dampings $\epsilon_{ \pm}(\vec{q})$ in Eq. (6.21) are $0\left(q^{2}\right)$, and given

$$
\begin{aligned}
\epsilon_{ \pm}(\vec{q})= & \pm \frac{c_{ \pm}\left(\theta_{\vec{q}}, \phi_{\vec{q}}\right)}{2 c_{2}\left(\theta_{\vec{q}}, \phi_{\vec{q}}\right)}\left[\Gamma_{v}(\vec{q})+\Gamma_{\rho}(\vec{q})\right] \\
& \mp \frac{v_{0} \cos \left(\theta_{\vec{q}}\right)}{2 c_{2}\left(\theta_{\vec{q}}, \phi_{\vec{q}}\right)}\left(\Gamma_{v}(\vec{q})+\frac{\gamma}{v_{0}} \Gamma_{\rho}(\vec{q})\right) .
\end{aligned}
$$

Note that, unlike the isotropic problem in $d>2$, here there are no transverse modes in any $d$ : we always have just two longitudinal Goldstone modes associated with $\delta \rho$ and $v_{x}$.

We can now again parallel our treatment of the isotropic model and calculate the correlation functions and propagators. The calculation is so similar that we will not repeat the details, but merely quote the results:

$$
\begin{gathered}
G_{v v}(\vec{q}, \omega)=\frac{i\left(\omega-v_{0} q_{y}\right)-\Gamma_{\rho}(\vec{q})}{\operatorname{Den}(\vec{q}, \omega)}, \\
G_{v \rho}(\vec{q}, \omega)=\frac{i \sigma_{1} q_{x}}{\operatorname{Den}(\vec{q}, \omega)}, \\
G_{\rho v}(\vec{q}, \omega)=\frac{i \rho_{0} q_{x}}{\operatorname{Den}(\vec{q}, \omega)}, \\
G_{\rho \rho}(\vec{q}, \omega)=\frac{i\left(\omega-\gamma q_{y}\right)-\Gamma_{v}(\vec{q})}{\operatorname{Den}(\vec{q}, \omega)}, \\
C_{v v}(\vec{q}, \omega)=\frac{\Delta\left[\left(\omega-v_{0} q_{y}\right)^{2}+\Gamma_{\rho}^{2}(\vec{q})\right]}{|\operatorname{Den}(\vec{q}, \omega)|^{2}},
\end{gathered}
$$

$$
\begin{aligned}
C_{\rho v}(\vec{q}, \omega) & =\left\langle\delta \rho(\vec{q}, \omega) v_{x}(-\vec{q},-\omega)\right\rangle \\
& =\frac{\Delta \sigma_{1} q_{x}\left[\omega-v_{0} q_{y}-i \Gamma_{\rho}(\vec{q})\right]}{|\operatorname{Den}(\vec{q}, \omega)|^{2}},
\end{aligned}
$$

and

$$
C_{\rho \rho}(\vec{q}, \omega)=\frac{\Delta \rho_{0}^{2} q_{x}^{2}}{|\operatorname{Den}(\vec{q}, \omega)|^{2}},
$$

where we have defined

$$
\begin{aligned}
\operatorname{Den}(\vec{q}, \omega)= & {\left[\omega-c_{+}\left(\theta_{\vec{q}}, \phi_{\vec{q}}\right) q\right]\left[\omega-c_{-}\left(\theta_{\vec{q}}, \phi_{\vec{q}}\right) q\right] } \\
& +i\left\{\omega\left[\Gamma_{\rho}(\vec{q})+\Gamma_{v}(\vec{q})\right]-q_{y}\left[v_{0} \Gamma_{v}(\vec{q})\right.\right. \\
& \left.\left.+\gamma \Gamma_{\rho}(\vec{q})\right]\right\}
\end{aligned}
$$

which, of course, implies

$$
\begin{aligned}
|\operatorname{Den}(\vec{q}, \omega)|^{2}= & {\left[\omega-c_{+}\left(\theta_{\vec{q}}, \phi_{\vec{q}}\right) q\right]^{2}\left[\omega-c_{-}\left(\theta_{\vec{q}}, \phi_{\vec{q}}\right) q\right]^{2} } \\
& +\left\{\omega\left[\Gamma_{\rho}(\vec{q})+\Gamma_{v}(\vec{q})\right]-q_{y}\left[v_{0} \Gamma_{v}(\vec{q})\right.\right. \\
& \left.\left.+\gamma \Gamma_{\rho}(\vec{q})\right]\right\}^{2} .
\end{aligned}
$$

These horrific expressions actually look quite simple when plotted as a function of $\omega$ at fixed $\vec{q}$; indeed, such a plot of $C_{v v}$ looks precisely like the solid line in Fig. 6: two asymmetrical peaks, centered at $\omega=c_{ \pm}\left(\theta_{q}, \phi_{q}\right) q$, with widths $\epsilon_{ \pm}(\vec{q}) \propto q^{2}$.

Note that, at this linear order, everything scales as it did in the isotropic problem: peak positions $\propto q$, widths $\propto q^{2}$, and heights $\propto 1 / q^{4}$.

Continuing to blindly follow the path we trod for the isotropic problem, we can calculate the equal-time $v_{x}-v_{x}$ correlation function:

$$
\begin{aligned}
C_{v v}(\vec{q}) & \equiv\left\langle v_{x}(\vec{q}, t) v_{x}(-\vec{q}, t)\right\rangle \\
& =\int_{-\infty}^{\infty} \frac{d \omega}{2 \pi} C_{v v}(\vec{q}, \omega)=\frac{\Delta}{2} \frac{\phi(\hat{q})}{\Gamma_{L}(\vec{q})}
\end{aligned}
$$

where $\phi(\hat{q})$ depends only on the direction $\hat{q}$ of $\vec{q}$, and is given by

$$
\begin{aligned}
\phi(\hat{q})= & \frac{1}{c_{2}\left(\theta_{\vec{q}}, \phi_{q}\right) q}\left[\frac{\left[c_{+}\left(\theta_{\vec{q}}, \phi_{\vec{q}}\right) q-v_{0} q_{y}\right]^{2}}{c_{+}\left(\theta_{\vec{q}}, \phi_{\vec{q}}\right) q-v_{0} q_{y}+\left[c_{+}\left(\theta_{\vec{q}}, \phi_{\vec{q}}\right) q-\lambda_{1} v_{0} q_{y}\right] \Gamma_{\rho} / \Gamma_{v}}\right. \\
& \left.+\frac{\left[c_{-}\left(\theta_{\vec{q}}, \phi_{\vec{q}}\right) q-v_{0} q_{y}\right]^{2}}{c_{+}\left(\theta_{\vec{q}}, \phi_{\vec{q}}\right) q-v_{0} q_{y}+\left[c_{-}\left(\theta_{\vec{q}}, \phi_{v e c}\right) q-\lambda_{1} v_{0} q_{y}\right] \Gamma_{\rho} / \Gamma_{v}}\right] .
\end{aligned}
$$


These fluctuations again diverge like $1 / q^{2}$ as $|\vec{q}| \rightarrow 0$, just as in the isotropic problem.

This completes our abbreviated discussion of the linearized theory of the anisotropic model. The most succinct summary of this linearized theory is that everything scales just as it did in the isotropic problem. This implies that the nonlinearities [i.e., the $\lambda$ and $\sigma_{2}$ terms in the equations of motion (6.15)] become relevant in and below the same upper critical dimension $d_{u c}=4$ as in the isotropic problem. For $d<4$, therefore, these nonlinearities will change the long-distance behavior of the anisotropic model. We will now treat these nonlinearities using renormalization-group arguments similar to those we used for the isotropic model in $d=2$. Now, however, they will work for all $d$ between 2 and 4 .

Notice that all of the nonlinearities in Eq. (6.15) are total $x$ derivatives, just as in the $d=2$ case for the isotropic problem. Now, however, this is true in all spatial dimensions, not just in $d=2$. (This, of course, is the reason we chose to consider precisely two "soft" components.) Thus, we will now be able to derive exact exponents in this model for all spatial dimensions. We will not go through the arguments in detail, as they are virtually identical to those in the $d=2$ case for the isotropic model, but will simply quote the conclusions:

(1) There are no graphical corrections to any of the diffusion constants in Eq. (6.15) except $D_{x}$.

(2) The stable fixed point that controls the ordered phase must have $\lambda_{\rho}^{*} \neq 0$ at least for $\lambda(0)<\lambda_{\rho}(0)$, which is a finite fraction of all flocks, and

(3) $\Delta$ and $\lambda_{\rho}$ are not graphically renormalized.

Point one suggests that, in constructing our dynamical renormalization group for Eq. (6.15), we should scale the $x$ direction differently from both the $y$ direction and the $d-2$ hard directions. Furthermore, since both the $y$ direction and the $d-2$ hard directions are alike in having their associated diffusion constants unrenormalized, we should scale these directions the same way. Therefore, in our renormalization group, we will rescale as follows: $x \rightarrow b x, \quad\left(y, \vec{x}_{H}\right)$ $\rightarrow b^{\zeta}\left(y, \vec{x}_{H}\right), t \rightarrow b^{z} t$. With these rescalings, the recursion relations for $D_{i}, i \neq x, \rho, \Delta$, and $\lambda_{\rho}$ become

$$
\begin{gathered}
\frac{d D_{i}}{d l}=(z-2 \zeta) D_{i} \quad(i \neq x), \\
\frac{d \Delta}{d l}=[z-2 \chi+(1-d) \zeta-1] \Delta, \\
\frac{d \lambda_{\rho}}{d l}=(\chi+z-1) \lambda_{\rho} .
\end{gathered}
$$

All three relations are exact, since none of these parameters experiences any graphical renormalization. As in the isotropic case, we want all of these parameters to flow to fixed points; this leads to three exact scaling relations between the three exponents $\chi, z$, and $\zeta$ :

$$
\begin{gathered}
z=2 \zeta, \\
z-2 \chi+(1-d) \zeta=1,
\end{gathered}
$$

$$
\chi=1-z,
$$

whose solution is easily found in all $d<4$ :

$$
\begin{aligned}
& \zeta=\frac{3}{7-d}, \\
& z=\frac{6}{7-d}, \\
& \chi=\frac{1-d}{7-d} .
\end{aligned}
$$

Note that these reduce to our isotropic results in $d=2$, as they should, since the two models are identical there. They also reduce to the harmonic values $z=2, \zeta=1$, and $\chi=-1$, in $d=4$, as they should, since 4 is the upper critical dimension.

In the physically interesting case of $d=3$, we obtain:

$$
\begin{gathered}
\zeta=\frac{3}{4}, \\
z=\frac{3}{2}, \\
\chi=-\frac{1}{2} .
\end{gathered}
$$

As in the isotropic case, we can use scaling arguments here to show that the effect of the nonlinearities can be fully incorporated by simply replacing $D_{x}$ everywhere it appears in the linearized expressions by the divergent, wave-vectordependent scaling form:

$$
D_{x}(\vec{q})=q_{x}^{z-2} f\left[\frac{\left(q_{y} / \Lambda\right)}{\left(q_{x} / \Lambda\right)^{\zeta}}, \frac{\left(q_{H} / \Lambda\right)}{\left(q_{x} / \Lambda\right)^{\zeta}}\right] .
$$

Doing this leads to all of the scaling laws for this anisotropic problem quoted in the Introduction.

\section{TESTING THE THEORY IN SIMULATIONS AND EXPERIMENTS}

In this section, we discuss how our theory can be tested in simulations and direct observations of real flocks. The "real" flocks may include, e.g., mechanical, self-propelled "go carts" packed so densely that they align with their neighbors [11], as well as aggregates of genuinely living organisms.

We begin with a few suggestions about the best boundary conditions and parameter values for simulations or experiments, and then describe how the correlation functions and scaling exponents $\chi, z$, and $\zeta$ predicted by our theory can be measured. The most useful boundary conditions are "torus" conditions; that is, reflecting walls in $d-1$ directions, and periodic boundary conditions in the remaining direction, call it $y$ (see Fig. 9). The advantage of these conditions is that one knows a priori that, if the flock does spontaneously order, its mean velocity will necessarily be in the periodic $(y)$ direction.

It might be objected that imposing such anisotropic boundary conditions breaks the rotation invariance our model requires, but this is not, in fact, the case. A "bird" 


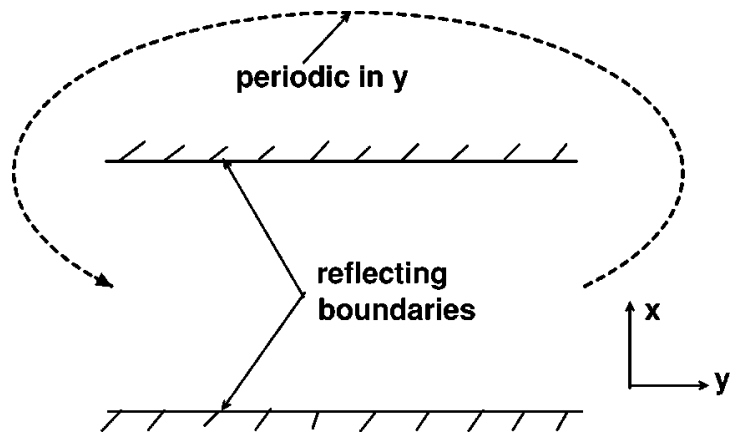

FIG. 9. Illustration of the optimal boundary conditions for simulations and experiments to test our predictions. The top and bottom walls are reflecting, while periodic boundary conditions apply at the left and right walls (i.e., a bird that flies out to the right instantly reappears at the same height on the left). The mean direction of spontaneous flock motion, if any occurs, is clearly forced to be horizontal by these boundary conditions. In spatial dimensions $d$ $>2$, one should choose reflecting boundary conditions in $d-1$ directions, and periodic in the remaining direction, thereby forcing $\langle\vec{v}\rangle$ to point along that periodic direction.

deep inside the box moves with no special direction picked out a priori; it can only find out about the breaking of rotation invariance on the boundary if the bulk of the flock spontaneously develops long-range order. This is precisely analogous to the way one speaks of a ferromagnet as spontaneously breaking a continuous symmetry even if it orders in the presence of ordered boundary conditions. So, by imposing these boundary conditions, we know the direction of the flock motion (the $y$ direction in the simulation), and, therefore, have oriented the simulation axes with the axes used in our theoretical discussion; i.e., our $\|$ axis equals the simulation's periodic direction.

Alternative boundary conditions add the further complication of having to first determine the direction of mean flock motion before calculating correlation functions. This complication is even worse for a finite flock (as any simulation must treat), since the mean direction of motion will wander, executing essentially a random walk that will explore the full circle in a time of order $T_{\text {flock }}=2 \pi \sqrt{N / \Delta}$. Our results, which assume a constant direction of flock motion, will only apply for time scales $t \ll T_{\text {Flock }}$. Even drifts of the mean flock direction through angles $\ll 2 \pi$ can cause problems, however, since most of the interesting scaling behavior is concentrated in a narrow window of angles $q_{\|} \sim q_{\perp}^{\zeta} \gg q_{\perp}$; i.e., near the direction of mean flock motion. So this drift greatly complicates the experimental analysis, and is best avoided by using the toroidal boundary conditions just described.

Of course, it is considerably harder to produce these boundary conditions in a real experiment. Ants walking around a cylinder may come close, although gravity will always break rotation invariance on a real cylinder. Perhaps the experiment could be done on the space shuttle, or with a rapidly spinning cylinder producing artificial gravity that swamps real gravity, or by using neutrally buoyant organisms in a fluid. Alternatively, one could use a "track" such as that shown in Fig. 10, and take data only from the crosshatched region, chosen to be in the middle of the straight section of the track, far from the curves.

Other, more ingenious ways to prepick the direction of

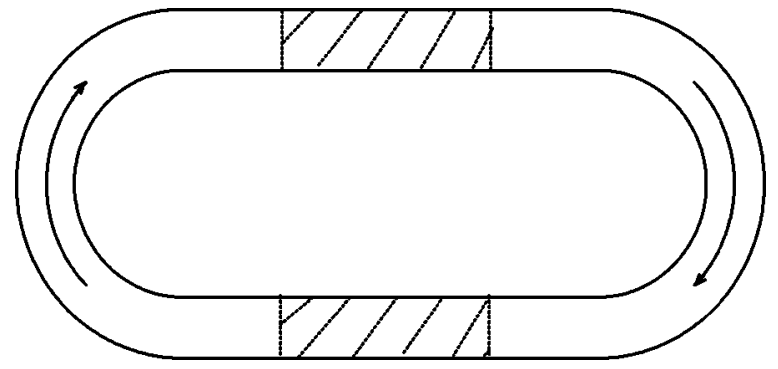

FIG. 10. More practical "track' geometry for experiments on real flocks. Data should only be taken from the cross-hatched region centered on the middle of the "straightaway."

mean motion through boundary conditions may also be dreamed up by experimentalists more clever than we are.

We strongly caution anyone attempting to test our results, however, that it is only through boundary conditions that one may prepick the direction of mean motion. Any approach that prepicks this direction in the bulk of the flock, such as giving each bird a compass, letting them be blown by a wind, or run downhill, or follow a chemical scent, etc., will lead to a model outside the universality class of our isotropic model, since the starting model does not have any rotation invariance to be spontaneously broken (unless the anisotropy leaves an "easy plane" in which all directions are equivalent, in which case our anisotropic model of Sec. VI applies). Indeed, such flocks of "birds with compasses" will be less interesting than the models we have studied here, since the "compass" will introduce a "mass" that makes any fluctuation away from the prepicked direction of flock motion decay rapidly (i.e., nonhydrodynamically) with time. In such a model, it is easy to show that the nonlinearities are irrelevant, and there are no interesting fluctuations left at long distances and times.

And now a few words about parameter choices. For definiteness, we will discuss in what follows the Vicsek model, whose parameters are $v_{0}=S / R_{0}$, where $S$ is the distance the birds travel on each time step and $R_{0}$ is the radius of the circle of neighbors, the mean number density $\rho_{0}$ in units of $1 / R_{0}^{d}$ where $d$ is the dimension of the system, and the noise strength $\Delta$, which is the mean squared angular error. Since the interesting nonlinear effects in our model come from terms proportional to $v_{0}^{2}$, those effects will become important at shorter length scales in a faster moving flock. That is, in, e.g., the Vicsek model, should we choose the dimensionless velocity as large as possible, consistent with the flock ordering. However, if we take $v_{0}$ too big, i.e., $v_{0} \gg 1$, then, on each time step, each bird is likely to have a completely different set of neighbors. It is difficult to see how order can develop in such a model. So, to take $v_{0}$ as big as possible without violating $v_{0} \gg 1$, we should choose $v_{0} \sim 1$. The simulations of Vicsek et al. [1] took $v_{0} \ll 1$, and, hence, probably never explored (in their finite flocks) the long length scale regime in which our nonlinear effects become important.

Now to the mean density $\rho_{0}$, which is, of course, just determined by the total number of birds $N$ and the volume $V$ of the box via $\rho_{0}=N / V$. We clearly want this to be large enough that each bird usually finds some neighbors in its neighbor sphere: This means we want $\rho_{0} R_{0}^{d} \geqslant O(1)$. How- 
ever, if we make $\rho_{0}$ too large, each bird has so many neighbors that a simulation is considerably slowed down, since the "direction picking" step of the Vicsek algorithm takes a time proportional to the number of neighbors (because we have got to average their directions). Thus, for simulations, one wishes to choose $\rho_{0}$ as small as possible, consistent, again, with getting good order.

Finally, we consider the noise $\Delta$. Here again, to see our fluctuation effects, we want $\Delta$ as big as possible. However, if $\Delta$ is too big, the flock will not order. Furthermore, even if $\Delta$ is small enough that the flock does order, we want also to be sure that we are well below the critical value $\Delta_{c}$ of $\Delta$ at which the flock disorders. Otherwise, for distances smaller than the correlation length $\xi$ associated with the orderdisorder transition, the scaling properties of the flock will be controlled by the fixed point that controls the order-disorder transition, not the low-temperature fixed point we have studied here.

If this transition is continuous, as it appears to be in Vicsek's simulations [1], this correlation length diverges as $\Delta$ $\rightarrow \Delta_{c}^{-}$. Thus, to observe scaling behavior we predict over as many decades of length scale as possible, we want to choose $\Delta$ substantially less than $\Delta_{c}$, but as big as possible consistent with this (to maximize fluctuation effects). Choosing $\Delta$ to be a little below the point at which the mean velocity $\langle\hat{v}\rangle$ starts to "saturate" seems like a fairly good compromise between these two competing effects. Similar considerations apply for choosing the optimal $\rho_{0}$, and $v_{0}$, which we want to be as small or big, respectively, as they can be without substantially suppressing long-ranged order. The best choices will probably lead to all three parameters $\rho_{0}, v_{0}$, and $\Delta$ being, in suitably dimensionless units, $O(1)$.

Having chosen the appropriate parameter values and boundary conditions, what should an experimentalist or simulator measure to test our theory? We have already discussed a number of such measurements in the Introduction; namely, the spatially Fourier-transformed equal-time and spatiotemporally Fourier-transformed unequal time densitydensity correlation functions $C \rho(\vec{q})$ and $C \rho(\vec{q}, \omega)$, respectively. Our predictions for these are given in Eqs. (5.38) and (5.40).

One additional correlation function that can be measured quite easily is the mean-squared lateral displacement of a bird:

$$
w^{2}(t) \equiv\left\langle\left|\vec{x}_{i}^{\perp}(t)-\vec{x}_{i}^{\perp}(0)\right|^{2}\right\rangle
$$

perpendicular to the mean direction of motion of the flock. This can easily be measured as a function of time in a simulation or experiment simply by labeling a set of $n$ birds in a "strip" near the center of the channel with its long axis running parallel to the mean direction of bird motion (see Fig. 11) and then following their subsequent motion. It is best to center the strip in the channel so as to postpone the birds reaching the reflecting walls as long as possible. Once they do reach the walls, of course $w^{2}(t \rightarrow \infty)$ saturates at $\sim L_{\perp}^{2}, L_{\perp}$ being the width of the channel. We will deal in the following discussion with times much smaller than that required for a bird at the center of the channel to wander out to its edge. Since the $\perp$ position $\vec{x}_{i}^{\perp}$ of each bird obeys
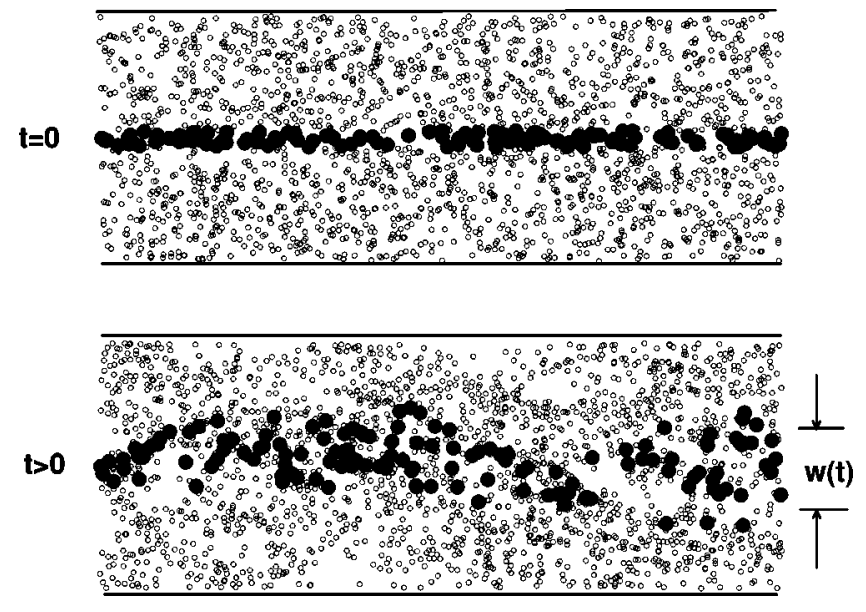

FIG. 11. Illustration of the experiment to measure the meansquared lateral wandering $w^{2}(t)$. One labels all of the birds some central stripe (of width $\ll L$, the channel width), and then measures the evolution of their mean displacements $\vec{x}_{\perp}(t)$ perpendicular to the mean direction of motion (which mean direction is horizontal in this figure).

$$
\vec{x}_{i}^{\perp}(t)=\vec{x}_{i}^{\perp}(0)+\int_{0}^{t} \vec{v}_{i}^{\perp}(t) d t
$$

where $\vec{v}_{i}^{\perp}(t)$ is the $\perp$ velocity of the $i$ th bird at time $t$, the mean width is given by

$$
w^{2}(t)=\int_{0}^{t} d t^{\prime} \int_{0}^{t} d t^{\prime \prime}\left\langle\vec{v}_{i}^{\perp}\left(t^{\prime}\right) \cdot \vec{v}_{i}^{\perp}\left(t^{\prime \prime}\right)\right\rangle
$$

Now we need to relate the velocity of the $i$ th bird to the position and time-dependent velocity field $\vec{v}_{\perp}(\vec{r}, t)$. This is easily done:

$$
\vec{v}_{i}^{\perp}(t)=\vec{v}_{\perp}\left[\vec{r}_{i}(t), t\right]
$$

where $\vec{r}_{i}(t)$ is the position of the $i$ th bird at time $t$. This is given by

$$
\vec{r}_{i}(t)=\vec{r}_{i}(0)+\bar{v} t \hat{x}_{\|}+\delta x_{i}^{\|}(t) \hat{x}_{\|}+\overrightarrow{\delta x}_{i}^{\perp}(t),
$$

where

$$
\bar{v} \equiv \frac{1}{N}\left|\sum_{i} \vec{v}_{i}\right|
$$

is the velocity averaged over all birds, which, as discussed earlier, is not to be confused with the space averaged $v_{0}$ $\equiv\left|\int \vec{v}(\vec{r}, t) d^{d} r\right|$ that appears in the expression for the sound speeds $c_{ \pm}\left(\theta_{q}\right)$. This distinction proves to be crucial here, as we shall see in a moment. In Eq. (7.5), $\delta x_{i}^{\|}(t)$ and $\delta x_{i}^{\perp}(t)$ reflect the motion of the $i$ th individual bird relative to the mean motion of the flock (at speed $\bar{v}$ ).

Using Eq. (7.5), we see that the desired single bird autocorrelation function in Eq. (7.3) is 


$$
\begin{aligned}
\left\langle\vec{v}_{i}^{\perp}\left(t^{\prime}\right) \cdot \vec{v}_{i}^{\perp}\left(t^{\prime \prime}\right)\right\rangle= & \left\langle\vec { v } _ { \perp } \left\{\vec{r}_{i}(0)+\left[\bar{v} t^{\prime}+\delta x_{\|}\left(t^{\prime}\right)\right] \hat{x}_{\|}\right.\right. \\
& \left.+\vec{\delta} x_{\perp}\left(t^{\prime}\right), t^{\prime}\right\} \cdot \vec{v}_{\perp}\left[\vec{r}_{i}(0)+\bar{v} t^{\prime \prime}+\delta x_{\|}\left(t^{\prime \prime}\right) \hat{x}_{\|}\right. \\
& \left.\left.+\overrightarrow{\delta x}_{\perp}\left(t^{\prime \prime}\right), t^{\prime \prime}\right]\right\rangle \\
= & C_{c}\left[\vec{x}_{\perp}\left(t^{\prime}\right)-\vec{x}_{\perp}\left(t^{\prime \prime}\right), \bar{v}\left|t^{\prime}-t^{\prime \prime}\right|\right. \\
& \left.+\delta x_{\|}\left(t^{\prime}\right)-\delta x_{\|}\left(t^{\prime \prime}\right), t^{\prime}-t^{\prime \prime}\right]
\end{aligned}
$$

where $C_{c}(\vec{R}, t)$ is the real-space velocity field autocorrelation function defined in the Introduction.

We assume (and will verify a posteriori) that both $\delta x_{\|}$ and $\vec{\delta} x_{\perp}$ are small enough compared to the average motion $\bar{v} t \hat{x}_{\|}$that their effect on the velocity-velocity autocorrelation in Eq. (7.7) is negligible. For now neglecting them, we see that we are left with the task of evaluating $C_{v}\left(\vec{R}_{\perp}=0, R_{\|}\right.$ $=\bar{v} t, t)$.

Expressing $C_{c}$ in terms of its Fourier transform then gives

$$
C_{c}\left(\vec{R}_{\perp}=0, R_{\|}=\bar{v} t, t\right)=\int d^{d-1} q_{\perp} d q_{\|} d \omega e^{i(\omega-\bar{v} q \| t)} C_{i i}(\vec{q}, \omega) .
$$

Using the fact that $C_{i i}(\vec{q}, \omega)$ is peaked at $\omega=c_{ \pm}\left(\theta_{\vec{q}}\right) q$ with widths that scale like $q_{\perp}^{z} f\left[q_{\|} \ell_{0} /\left(q_{\perp} \ell_{0}\right)^{\zeta}\right]$, the dominating peak is at $\omega=\omega_{-}$for $v_{0}(0)>\gamma(0)$ or at $\omega=\omega_{+}$for $v_{0}(0)$

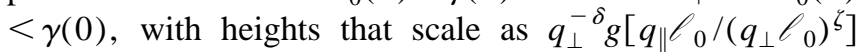
with $\delta=2 \chi+z+\zeta+d-1$ [see Eq. (5.56)]. Assuming that $v_{0}(0)>\gamma(0)$, it is straightforward to show that, upon integrating Eq. (7.8) over $\omega$, we obtain

$$
C_{c}=\int d^{d-1} q_{\perp} d q_{\|} e^{i\left[c_{-}\left(\theta_{q}^{-}\right) q-\bar{v} q_{\|}\right] t} f_{-}\left(\frac{q_{\|} \ell_{0}}{\left(q_{\perp} \ell_{0}\right)^{\zeta}}\right) q_{\perp}^{z-\delta} .
$$

This integral is dominated, as $t \rightarrow \infty$, by $q_{\|} \sim\left(q_{\perp} \ell_{0}\right)^{\zeta} / \ell_{0}$ $\gg q_{\perp}$; hence, $\theta_{\vec{q}} \rightarrow 0$, and we get

$$
C_{c}=\int d^{d-1} q_{\perp} d q_{\|} e^{i\left(v_{0}-\bar{v}\right) q_{\|} t} f_{-}\left(\frac{q_{\|} \ell_{0}}{\left(q_{\perp} \ell_{0}\right)^{\zeta}}\right) q_{\perp}^{z-\delta} .
$$

We can scale the time dependence out of this integral with the change of variables

$$
q_{\|} \equiv \frac{Q_{\|}}{t} \quad \text { and } \quad \vec{q}_{\perp} \equiv \frac{\vec{Q}_{\perp}}{t^{(1 / \zeta)}},
$$

which give

$$
C_{c} \propto t^{2 \chi / \zeta} .
$$

Using this in Eq. (7.7) for the single bird velocity autocorrelation function, and then using that autocorrelation function in the expression (7.3) for the mean squared random walk distance gives

$$
w^{2}(t) \propto \int_{0}^{t} d t^{\prime} \int_{0}^{t} d t^{\prime \prime}\left|t^{\prime}-t^{\prime \prime}\right|^{2 \chi / \zeta} .
$$

Now, we need to distinguish two cases:

Case (1): $2 \chi / \zeta>-1$. In this case, which holds in $d=2$, where $\chi=-\frac{1}{5}$ and $\zeta=\frac{3}{5}$, the double integral over $t^{\prime}$ and $t^{\prime \prime}$ is dominated, for $t \gg t_{0}$, the microscopic time scale, by $t^{\prime}, t^{\prime \prime}$, and $\left|t^{\prime}-t^{\prime \prime}\right|$ of order $t \gg t_{0}$. Hence, our calculation of $C_{c}$ which used the hydrodynamic (i.e., long time) limiting forms of the correlation functions, is correct, and Eq. (7.13) holds. Changing variables to $T^{\prime} \equiv t^{\prime} / t$ and $T^{\prime \prime} \equiv t^{\prime \prime} / t$, we see that

$$
w^{2}(t) \propto t^{2(1+\chi / \zeta)}=t^{4 / 3}, \quad \frac{2 \chi}{\zeta}>-1,
$$

the last equality holding in $d=2$. Note that this behavior is "hyperdiffusive": the mean-squared displacement $w^{2}(t)$ grows faster than it would in a simple random walk; i.e., faster than linearly with time $t$.

Case (2): $2 \chi / \zeta<-1$. In this case, which certainly holds for $d>4$ (where $\chi=1-d / 2<-1$ and $\zeta=1$ ), the integral over $t^{\prime \prime}$ converges as $\left|t^{\prime}-t^{\prime \prime}\right| \rightarrow \infty$. Hence, that integral is, in fact, dominated by $\left|t^{\prime}-t^{\prime \prime}\right|=O\left(t_{0}\right)$, the microscopic time, where our hydrodynamic result Eq. (7.12) is not valid. Presumably, the correct $\left|t^{\prime}-t^{\prime \prime}\right| \rightarrow 0$ limit of the single bird velocity autocorrelation (7.3) is finite; and, hence, so the integral over $t^{\prime \prime}$ in Eq. (7.13) approaches a finite limit as $t \rightarrow \infty$.

Hence, we get

$$
w^{2}(t) \propto \int_{0}^{t} d t^{\prime} \times(\text { finite constant }) \propto t, \quad \frac{2 \chi}{\zeta}<-1 .
$$

We now need only verify our a posteriori assumptions that $\delta x_{\|}$and $\vec{\delta} x_{\perp}$ were negligible in the velocity-velocity autocorrelation Eq. (7.7).

First consider $\vec{x}_{\perp}$; we have just shown that the root-meansquared $\left|\vec{x}_{\perp}\left(t^{\prime}\right)-\vec{x}_{\perp}\left(t^{\prime \prime}\right)\right| \propto\left|t^{\prime}-t^{\prime \prime}\right|^{1+\chi / \zeta}$. From our scaling expression (6.43), we see that $C_{c}\left(R_{\perp}, R_{\|}, t\right) \approx C_{c}\left(R_{\perp}\right.$ $\left.=0, R_{\|}, t\right)$ if $R_{\perp}^{\zeta} \ll R_{\|}$. In Eq. (7.7), we are interested in $R_{\perp}$ $\propto\left|t^{\prime}-t^{\prime \prime}\right|^{1+\chi / \zeta}$ and $R_{\|} \propto\left|t^{\prime}-t^{\prime \prime}\right|$; hence, the condition $R_{\perp}^{\bar{\zeta}}$ $\ll R_{\|}$will be satisfied as $\left|t^{\prime}-t^{\prime \prime}\right| \rightarrow \infty$ provided $\zeta+\chi<1$. Since $\zeta \leqslant 1$ and $\chi<0$ for all $d \geqslant 2$, this condition is satisfied for all $d \geqslant 2$. For $\delta x_{\|}$we need only show that $\mid \delta x_{\|}\left(t^{\prime}\right)$ $-\delta x_{\|}\left(t^{\prime \prime}\right)|\ll| t^{\prime}-t^{\prime \prime} \mid$ as $\left|t^{\prime}-t^{\prime \prime}\right| \rightarrow \infty$. This is easily shown by using the fact, alluded to earlier, that $\delta v_{\|}(\vec{r}, t)$, the fluctuation of the velocity along the mean direction of motion, has only short-ranged temporal correlations. Using this fact, it is straightforward to show that $\delta x_{\|}(t)$ just executes a simple random walk; that is,

$$
\sqrt{\left|\delta x_{\|}\left(t^{\prime}\right)-\delta x_{\|}\left(t^{\prime \prime}\right)\right|^{2}} \propto \sqrt{\left|t^{\prime}-t^{\prime \prime}\right|} \ll\left|t^{\prime}-t^{\prime \prime}\right|
$$

and hence these fluctuations are negligible as well.

Unfortunately, the analogous calculation for the anisotropic model shows that this random "transverse walk" is much less interesting: the mean-squared transverse displacement in the $x$ direction (the direction in the "easy place" of the anisotropic model orthogonal to the mean direction of motion, $y$ ) is given by an expression very similar to Eq. (7.16) 


$$
\left\langle\left|x_{i}(t)-x_{i}(0)\right|^{2}\right\rangle \equiv w^{2}(t)=\int_{0}^{t} d t^{\prime} \int_{0}^{t} d t^{\prime \prime}\left\langle v_{i x}\left(t^{\prime}\right) v_{i x}\left(t^{\prime \prime}\right)\right\rangle
$$

and a calculation so closely analogous to that just given for the isotropic model that we shall not bother to repeat it for this case shows that

$$
\left\langle v_{i x}\left(t^{\prime}\right) v_{i x}\left(t^{\prime \prime}\right)\right\rangle \propto\left|t^{\prime}-t^{\prime \prime}\right|^{3-d-2 / z} .
$$

As in our analysis of the isotropic case, here, too, the question of whether "simple random walk" behavior $\left[w^{2}(t)\right.$ $\propto t]$ or "hyperdiffusive" behavior $\left[w^{2}(t) \propto t^{\gamma}, \gamma>1\right]$ occurs hinges entirely on whether the exponent in Eq. (7.18) is greater or less than -1 , with hyperdiffusive behavior occurring in the former case (exponent $>-1$ ) and simple random walk behavior in the latter (exponent $<-1$ ). Using our exact result (6.43) for $z$ in the anisotropic model for $2 \leqslant d \leqslant 4$, we see that hyperdiffusive behavior will occur if

$$
3-d-\frac{2}{z}=\frac{2-2 d}{3}>-1,
$$

which is satisfied only for $d<5 / 2$. Unfortunately, this condition is not satisfied for either $d=3$ or $d=4$. In $d=2$, the anisotropic model is the same as the isotropic model, while for $d>4, z=2$ and $3-d-2 / z<-1$. So in no case in which the anisotropic model is different from the isotropic one is hyperdiffusive behavior observable; rather, we expect $w^{2}(t) \propto t$ for all those cases. This negative prediction could be checked experimentally, although its confirmation, while a nontrivial check of our theory, would clearly be less exciting than verification of our hyperdiffusive prediction $w^{2}(t)$ $\propto t^{4 / 3}$ for the isotropic $d=2$ model.

Some of the numerical tests discussed in this section have been carried out recently, and good agreement with our prediction has been reached [12].

\section{FUTURE DIRECTIONS}

In this paper, we have only scratched the surface of a very deep and rich new subject. We have deliberately focused on the most limited possible question: what are the properties of a flock far from its boundaries, and deep within its ordered state? Every move away from these restricting simplifications opens up new questions. To name a few that we hope to address in the coming millennium:

(1) The transition from the ordered (moving) to disordered (stationary, on average) phase of the flock. This can be studied by analyzing the (unstable) fixed point at which the renormalized $\alpha$ of our original model (2.6) is zero. The dynamical RG analysis of this point would be technically similar to the one we have presented here for the low-temperature phase, with a few crucial differences: (a) All components of $\vec{v}$, not just the $\perp$ components, become massless at the transition. (b) The fixed point will be isotropic, since no special directions are picked out by $\langle\vec{v}\rangle$, since $\langle\vec{v}\rangle$ still $=0$ at the transition. (c) The $\beta|\vec{v}|^{2} \vec{v}$ term becomes another relevant vertex. We know, by power counting, that at the transition, this vertex becomes relevant in $d=4$. Indeed, if we ignore the $\lambda$ vertices, our model simply reduces to a purely relax- ational time-dependent Ginsburg Landau (TDGL) model for a spin system with the number of components $n$ of the spin equal to the dimension $d$ of the space those spins live in.

We have convinced ourselves by power counting that $a t$ the transition, for $d<4$, the $\lambda$ vertices are a relevant perturbation to the Gaussian critical point. Whether they constitute a relevant perturbation to the 4- $\epsilon$ TDGL fixed point, thereby changing its critical properties, can only be answered by a full-blown dynamical renormalization group analysis. Obviously, a similar analysis could also be done for the anisotropic model.

(2) The shape and cohesion of an open flock, and its fluctuations. We have thus far focused on flocks in closed or periodic boundary conditions. Real flocks are usually surrounded by open space. How do they stay together under these circumstances? What shape does the flock take? How does this shape fluctuate, and is it stable? This issue is somewhat similar to the problems of the shapes of equilibrium and growing crystals (e.g., faceting, dendritic growth). In those problems, it was important to first understand bulk processes (e.g., thermal diffusion in the case of dendritic growth) before one could address surface questions (e.g., dendritic growth). The nontrivial aspects of the bulk processes in flocks (e.g., anomalous diffusion) will presumably radically alter the shapes and their fluctuations.

(3) A somewhat related question is: What happens if birds move at different speeds? By "move at different speeds," we do not mean simply that at any instant, different birds will be moving at different speeds [a possibility already included in our "soft spin" dynamical model equation (2.6)]. Rather, we mean a model in which some birds have a different probability distribution of speeds than others. (In our model, this distribution of the speed of any given bird is the same over a sufficiently long time, and controlled by the values of the parameters $\alpha$ and $\beta$, with large $\alpha$ and $\beta$ leading to a distribution sharply peaked around a mean speed $v_{0}=\sqrt{\alpha / \beta}$, while small $\alpha$ and $\beta$ lead to a broader distribution). More generally, one could imagine two (or many) different species of birds, (labeled by $k$ ) all flying together, each with different mean speeds $v_{0}^{k}$. What would the bulk dynamics of such a flock be? Would there be large scale spatial segregation, with fast birds moving to the front of the flock, and slow birds moving to the back? If so, how would such segregation affect the shape of the flock? Would it elongate along the mean direction of motion? Would this elongation eventually split the flock into fast and slow moving flocks?

(4) At the other extreme, one could consider flocks in confined geometries; e.g., inside a circular reflecting wall in $d=2$. In such a case, the time averaged velocity of the flock $\langle\hat{v}(\vec{r}, t)\rangle_{t}$ could not be spatially homogeneous but would have to circulate around the center of the circle; i.e., $\langle\hat{v}(\vec{r}, t)\rangle_{t}=f(r) \hat{\theta}$. The spatially inhomogeneous pattern of velocity and density that resulted could be predicted by our continuum equations. This problem is potentially related to the previous one, since one way a flock containing, say, some very fast birds and other very slow birds, could stay together would be for the fast birds to fly in circles inside the essentially stationary volume of space filled by the slow birds. It would be very interesting to make the connection 
between our continuum theory and the recently observed circular motion of Dictyostelium cells in a confined geometry [13].

(5) One could relax the constraint on conservation of bird number, by allowing birds to be born, and die, "on the wing." Numerical studies of such models, which may be appropriate to bacteria colonies, where reproduction and death are rapid, as well as the migration of, e.g., huge herds of caribou over thousands of miles and many months, have already been undertaken [14]; it should be straightforward to modify our equations by adding a source term to the bird number conservation equation.

(6) It is possible that phase transitions other than that from the moving to the nonmoving state occur in flocks. For example, in some preliminary simulations of microscopic models in which birds try to avoid getting too close to their neighbors, rather than merely following them, we have observed (literally by eye) what appears to be a "flying crystal'" phase of flocks: the birds appear to lock themselves onto the sites of a crystalline lattice, which then appears to move coherently. It would be very interesting to test numerically whether this optical appearance reflects true long-range translational order, by looking for a nonzero expectation value of the translational order parameters.

$$
\rho_{\vec{G}}(t)=\left\langle\Sigma_{i} e^{i \vec{G} \cdot \vec{r}_{i}(t)} / N\right\rangle,
$$

which will become nonzero in the thermodynamic $(N \rightarrow \infty)$ limit at a set of reciprocal lattice vectors $\vec{G}$ if such longranged order actually develops. It will also be extremely interesting to include the possibility of such long-ranged order in our analytic model, and study the interplay between this translational order and the anomalous hydrodynamics that we have found here for "fluid" flocks. Will anomalous hydrodynamics suppress the "Mermin-Wagner" fluctuations of translational order, just as it does those of orientational order, and lead to true long-ranged translational order, even in $d=2$ ? Will the crystallization suppress orientational fluctuations, and thereby slow down the anomalous diffusion that we found in the fluid case? And in any case, what are the temporal fluctuations of $\rho_{\vec{G}}(t)$ ?

It should be noted that this problem potentially has all the richness of liquid crystal physics: in addition to "crystalline', phases, in which the set $\{\vec{G}\}$ of reciprocal lattice vectors in Eq. (8.1) spans all $d$ dimensions of space, one could imagine "smectic", phases in which all the $\vec{G}$ 's lay in the same direction, and "discotic" phases in $d=3$, in which the $\vec{G}$ 's only spanned a two-dimensional subspace of this threedimensional space. The melting transitions between these phases and the "fluid," moving flock, as well as possible direct transitions between them and the stationary flock phase, and between each other, would also be of great interest.

We should point out here that these models differ considerably from recently considered models of moving flux lattices [15] and transversely driven charge density waves $[15,16]$ in that here the direction of motion of the lattice is not picked out by an external driving force, but, rather, represents a spontaneously broken continuous symmetry.

(7) Finally, we would like to study the problem of the growth of order in flocks. This is a phenomenon we have all seen every time we walk onto a field full of geese: eventually, our approach startles the geese, and they take off en masse. Initially, they fly in random directions, but quickly the flock orders, and flies away coherently. The dynamics of this process is clearly in many ways similar to, e.g., the growth of ferromagnetic order after a rapid quench from an initial high temperature $T_{i}>T_{c}$, the Curie temperature, to a final temperature $T_{f}<T_{c}$, a problem that has long been studied [17] and proven to be very rich and intriguing. In flocks, where, as we have seen, even the dynamics of the completely ordered state is very nontrivial, the growth of order seems likely to be even richer.

Even this list of potential future problems, representing, as it does, probably another ten years of research for several groups, clearly represents only a narrow selection of the possible directions in which this embryonic field can go. We have not even mentioned, for example, the intriguing problem of one-dimensional flocking, with its applications to traffic flow (and traffic jams), a topic clearly of interest. This problem has recently been studied [18] and found to also show a nontrivial phase transition between moving and nonmoving states.

We expect flocking to be a fascinating and fruitful topic of research for biologists, computer scientists, and both experimental and theoretical physicists (at least these two) for many years to come.

\section{ACKNOWLEDGMENTS}

We profusely thank T. Vicsek for introducing us to this problem. We are also grateful to A. Csirok, E. V. Albano, and A. L. Barabasi for communicating their work to us prior to publication, to M. Ulm and S. Palmer for performing some inspirational simulations, and for equally inspirational discussions, to J. Sethna and K. Dahmen for pointing out the existence of the $\lambda_{2}$ and $\lambda_{3}$ terms, and to P. McEuen for suggesting the go-carts. J.T. thanks the Aspen Center for Physics for their hospitality at several stages of this work. J.T.'s work was supported in part by the National Science Foundation under Grant No. DMR-9634596.
[1] T. Vicsek, Phys. Rev. Lett. 75, 1226 (1995); A. Czirok, H. E. Stanley, and T. Vicsek, J. Phys. A 30, 1375 (1997).

[2] B. L. Partridge, Sci. Am. 246(6), 114 (1982).

[3] C. Reynolds, Comput. Graph. 21, 25 (1987); J. L. Deneubourg and S. Goss, Ethology, Ecology, Evolution 1, 295 (1989); A. Huth and C. Wissel, in Biological Motion, edited by W. Alt and E. Hoffmann (Springer-Verlag, Berlin, 1990), pp. 577590. We thank D. Rokhsar for calling these references to our attention.

[4] J. Toner and Y. Tu, Phys. Rev. Lett. 75, 4326 (1995).

[5] N. D. Mermin and H. Wagner, Phys. Rev. Lett. 17, 1133 (1966). 
[6] D. Forster, D. R. Nelson, and M. J. Stephen, Phys. Rev. A 16, 732 (1977).

[7] Smectic- $A$ liquid crystals show even stronger damping at long wavelengths than that found here for flocks. For a theoretical treatment, see G. F. Mazenko, S. Ramaswamy, and J. Toner, Phys. Rev. Lett. 49, 51 (1982); Phys. Rev. A 28, 1618 (1983). Experimental confirmation of this theory is given by S. Bhattacharya and J. B. Ketterson, Phys. Rev. Lett. 49, 997 (1982). See also the discussion in P. G. deGennes and J. Prost, The Physics of Liquid Crystals, 2nd ed. (Clarendon Press, Oxford, 1993), pp. 457-465.

[8] We thank Karen Dahmen and Jim Sethna for pointing out the existence of this term to us (although, given all the difficulty this term has caused us, it is unclear whether thanks are really the appropriate response).
[9] See, e.g., M. Kardar, G. Parisi, and Y.-C. Zhang, Phys. Rev. Lett. 56, 889 (1986).

[10] We thank Stephanie Palmer for suggesting this alternative approach to us.

[11] We thank Paul McEuen for suggesting this realization to us.

[12] Y. Tu, J. Toner, and M. Ulm, Phys. Rev. Lett. 80, 4819 (1998).

[13] H. Levine (private communication).

[14] E. V. Albano, Phys. Rev. Lett. 77, 2129 (1996).

[15] L. Balents, M. C. Marchetti, and L. Radzihovsky, Phys. Rev. Lett. 78, 751 (1997).

[16] L. Radzihovsky and J. Toner (unpublished).

[17] See, e.g., M. Mondello and N. Goldenfeld, Phys. Rev. E 47, 2384 (1993).

[18] A. Czirok, A. L. Barabasi, and T. Vicsek (unpublished). 